\title{
Resonant heavy Higgs searches at the HL-LHC
}

\author{
Amit Adhikary, ${ }^{a}$ Shankha Banerjee, ${ }^{b}$ Rahool Kumar Barman ${ }^{a}$ \\ and Biplob Bhattacherjee ${ }^{a}$ \\ ${ }^{a}$ Centre for High Energy Physics, Indian Institute of Science, \\ Bangalore 560012, India \\ ${ }^{b}$ Institute for Particle Physics Phenomenology, Department of Physics, Durham University, \\ Durham DH1 3LE, United Kingdom \\ E-mail: amitadhikary@iisc.ac.in, shankha.banerjee@durham.ac.uk, \\ rahoolbarman@iisc.ac.in, biplob@iisc.ac.in
}

ABSTRACT: In this work, we show the importance of searches for heavy resonant scalars $(H)$ and pseudoscalars $(A)$. Taking cue from the present searches, we make projections for searches in an extended scalar sector at the high luminosity run of the Large Hadron Collider. We study the three most relevant search channels, i.e., $H \rightarrow h h, H / A \rightarrow t \bar{t}$ and $b \bar{b} H / A$. Upon studying multifarious final states for the resonant double Higgs production, we find that the $b \bar{b} \gamma \gamma\left(\sigma(p p \rightarrow H \rightarrow h h) \in[81.27,14.45] \mathrm{fb}\right.$ for $m_{H} \in[300,600] \mathrm{GeV}$ at $95 \%$ C.L.) and $b \bar{b} b \bar{b}\left([5.4,2.5] \mathrm{fb}\right.$ for $m_{H} \in[800,1000] \mathrm{GeV}$ at $95 \%$ C.L.) channels are the most constraining. For the $b \bar{b} H$ channel, we can exclude $\sigma(p p \rightarrow b \bar{b} H) \in[22.2,3.7] \mathrm{fb}$ for $m_{H} \in[300,500] \mathrm{GeV}$. Finally, we consider the phenomenological Minimal Supersymmetric Standard Model as an example and impose various present constraints and our future direct search-limits and obtain strong constraints on the $m_{A}-\tan \beta$ parameter space, where $m_{A}$ and $\tan \beta$ are respectively the mass of the pseudoscalar and the ratio of the vacuum expectation values of the two Higgs doublets. Assuming that the heavy Higgs boson decays only to Standard Model (SM) states, we find that the $H \rightarrow h h \rightarrow b \bar{b} \gamma \gamma$ $(H \rightarrow t \bar{t})$ channel excludes $\tan \beta$ as low as $4\left(m_{A} \in[400,800] \mathrm{GeV}\right)$ at $95 \% \mathrm{CL}$. This weakens up to $\sim 5.5$ when the $b \bar{b} H$ channel dominates. Upon allowing for non-SM decay modes, the limits weaken.

Keywords: Beyond Standard Model, Higgs Physics, Supersymmetric Standard Model

ARXiv EPrint: 1812.05640 


\section{Contents}

1 Introduction 1

2 The $p p \rightarrow H \rightarrow h h$ channel $\quad 4$

2.1 The $b \bar{b} \gamma \gamma$ channel 4

2.1.1 The $p p \rightarrow A \rightarrow Z h$ channel $\quad 11$

2.2 The $b \bar{b} b \bar{b}$ channel 11

$\begin{array}{ll}2.3 \text { The } b \bar{b} \tau \tau \text { channel } & 17\end{array}$

2.3.1 The $b \bar{b} \tau_{h} \tau_{h}$ channel 18

$\begin{array}{ll}2.4 \text { The } b \bar{b} W W^{*} \text { channel } & 19\end{array}$

2.4.1 The $2 \ell 2 b+\mathbb{E}_{T}$ channel 22

2.4.2 The $1 \ell 2 b 2 j+\mathbb{E}_{T}$ channel 23

2.5 The $\gamma \gamma W W^{*}$ channel 25

2.5.1 The $\gamma \gamma 1 \ell 2 j+\mathbb{E}_{T}$ channel 28

2.5.2 The $\gamma \gamma 2 \ell+\mathbb{E}_{T}$ channel 28

2.6 Summarising the $H \rightarrow h h$ channel 30

3 The $p p \rightarrow H \rightarrow t \bar{t}$ channel

3.1 The leptonic channel 33

3.2 The semi-leptonic channel 33

4 The $(H / A) b \bar{b}$ channel $\quad 37$

4.1 The $\tau_{h} \tau_{h}$ channel: $b$-tag category 39

$5 \quad$ The future of the pMSSM parameter space $\quad 41$

$\begin{array}{lll}6 & \text { Summary } & 48\end{array}$

A Detailing the cross section with generation cuts for the signal and $\begin{array}{ll}\text { backgrounds } & 50\end{array}$

B Validation of the $b \bar{b} H$ analysis $\quad 51$

\section{Introduction}

The Higgs boson discovered in 2012, was the last missing piece in the Standard Model of particle physics (SM). The SM, however, is inadequate to explain the nature and existence of dark matter, the small but non-negligible masses of the neutrinos, the asymmetry between baryons and anti-baryons, to name a few. Besides, SM can not explain the hierarchy problem which is inherent in the theory. Well motivated theories including supersymmetry 
have the potential to solve some of these limitations. There are additional fundamental theoretical requirements that the SM can not satisfy. The aforementioned experimental observations and theoretical requirements compel us to look for physics beyond the Standard Model (BSM). However, the possibilities being innumerable, it is extremely difficult to ascertain the nature of such new physics. Since the discovery of the Higgs boson, and a growing convergence of its properties with the SM expectations [1-16], the new physics possibilities are gradually getting strongly constrained. Searches for BSM are being performed at the Large Hadron Collider (LHC) by the CMS, ATLAS, ALICE and LHCb collaborations. Except for some excitement in the flavour physics sector, there have not been any strong hints for new physics in the form of new particles or significant deviations in couplings with respect to the SM. Even though supersymmetry is perhaps one of the most elegant theories of our time, it comes with additional new particles, which need to be discovered at some stage. Even though searches are being conducted for a considerable region of parameter space for the Minimal Supersymmetric Standard Model (MSSM), there are more non-traditional searches which need to be performed. The MSSM parameter space has been extensively studied in light of the constraints from cosmology, flavour physics, and Run-I plus Run-II data from LHC [17-41]. However, there are simple extensions of the MSSM that can weaken the present bounds considerably. On the positive side, the LHC can potentially pin down the Higgs couplings to weak bosons and most of the fermions at the level of $\mathcal{O}(5-10 \%)$ [42-45]. However, as has been shown in numerous experimental [46-59] (including future extrapolations [60-62]) and phenomenological studies [63-72, 72-83, 83, 84, 84-107], the measurement of the elusive triple Higgs coupling $\left(\lambda_{h h h}\right)$ is a difficult feat at the LHC. Studies show that future colliders are expected to be more adept in constraining or even measuring this coupling to a great precision $[89,108-117]$. In order to be completely sure whether or not there is any extended Higgs sector, it is of utmost importance to measure the Higgs quartic coupling, $\lambda_{h h h h}$ and the Higgs trilinear coupling, $\lambda_{h h h}=\lambda_{h h h h} v$, where $v$ is the vacuum expectation value of the SM Higgs boson. Now, independent measurements of the Higgs couplings to the gauge bosons and fermions will constrain $v$ and we already have a precise Higgs mass measurement. To confirm this sector of the SM, one needs to measure $\lambda_{h h h}$ or $\lambda_{h h h h}[64,118-122]$.

In the following sections, we focus on the various production and decay processes of a resonant scalar, viz., a resonant decay to a pair of SM-like Higgs bosons, to a pair of top quarks, a heavy pseudoscalar, $A$, decaying to an SM-like Higgs boson and a $Z$ boson and the production of a heavy scalar in association with a pair of bottom quarks. The final theme of this work is in the context of the phenomenological MSSM (pMSSM). However, our results are presented in such a way that they can be mapped onto most models with an extended scalar sector. Table 1 summarises the various bounds set on the double-Higgs production cross-section by CMS and ATLAS in the non-resonant and resonant categories. The resonant production results are mostly interpreted in terms of spin-0 and spin-2 hypotheses. Besides, there are many supersymmetric interpretations for the resonant scalar searches. As an example, for the $b \bar{b} \tau^{+} \tau^{-}$resonant search performed by CMS [123], the MSSM parameters $m_{A}$ (mass of the $C P$-odd scalar, $A$ ) and $\tan \beta$ (ratio of the vacuum expectation values of the two Higgs doublets in the model, viz., $H_{u}$ and $H_{d}$ ) 


\begin{tabular}{|c|c|c|c|c|}
\hline Channel & $\begin{array}{c}\text { CMS (NR) } \\
(\times \mathrm{SM})\end{array}$ & $\begin{array}{c}\text { CMS (R) } \\
{[\mathrm{fb},(\mathrm{GeV})]}\end{array}$ & $\begin{array}{c}\text { ATLAS (NR) } \\
(\times \mathrm{SM})\end{array}$ & $\begin{array}{c}\text { ATLAS (R) } \\
{[\mathrm{fb},(\mathrm{GeV})]}\end{array}$ \\
\hline $\begin{array}{c}b \bar{b} b \bar{b} \\
{[133-135]}\end{array}$ & 75 & $\begin{array}{c}1500-45 \\
(260-1200)\end{array}$ & 13 & $\begin{array}{c}2000-2 \\
(260-3000)\end{array}$ \\
\hline $\begin{array}{c}b \bar{b} \gamma \gamma \\
{[136-138]}\end{array}$ & 24 & $\begin{array}{c}240-290 \\
(250-900)\end{array}$ & 22 & $\begin{array}{c}1100-120 \\
(260-1000)\end{array}$ \\
\hline $\begin{array}{c}b \bar{b} \tau^{+} \tau^{-} \\
{[59,123]}\end{array}$ & 30 & $3110-70$ & 12.7 & $1780-100$ \\
& & $(250-900)$ & & $(260-1000)$ \\
\hline$\gamma W W^{*}[139]$ & & & 200 & $40000-6100$ \\
$(\gamma \gamma \ell \nu j j)$ & & $20500-800$ & 300 & $(260-500)$ \\
\hline$b \bar{b} \ell \nu \ell \nu$ & 79 & $(300-900)$ & & $(500-3000)$ \\
{$[140,141]$} & & & 160 & $9300-2800$ \\
\hline$W W^{*} W W^{*}$ & & & & $(260-500)$ \\
{$[142]$} & & & & \\
\hline
\end{tabular}

Table 1. Bounds obtained on the di-Higgs cross-sections (in $\mathrm{fb}$ ) from CMS and ATLAS studies dedicated to the search for non-resonant (NR) and resonant (R) double Higgs production in various channels. The numbers in brackets show the range of the heavy scalar mass considered in that particular study.

are excluded in the range $230 \mathrm{GeV}<m_{A}<360 \mathrm{GeV}$ and $\tan \beta \lesssim 2$, at 95\% CL. Thus, besides measuring deviations to the Higgs self-coupling, there are other possible channels to look for in order to establish an extended scalar sector. Some of these new channels include the production of the SM-like Higgs in association with a $Z$-boson reconstructing a resonance. Another possible channel is the production of a pair of top-quarks. Now, the first of these channels can be via a heavy pseudoscalar resonance [124, 125], whereas the $t \bar{t}$ production can be either through a heavy scalar or pseudoscalar [126, 127]. However, both these channels can also come about from a heavy $Z^{\prime}[125,128-131]$. Now, in order to be sure whether the $Z h$ or $t \bar{t}$ production is via a spin- 0 or spin- 1 resonance, one needs to delve deeper into the angular observables. Lastly, we also study the effects of the high $\tan \beta$ regime for a heavy scalar produced in association with a pair of $b$-quarks and decaying to a pair of $\tau$-leptons [132].

Our paper is organised as follows. We study the reach of the HL-LHC for the $H \rightarrow h h$ channel in various final states, in section 2, by showing the 95\% CL bounds on $\sigma(p p \rightarrow$ $H \rightarrow h h)$ as functions of the heavy Higgs mass, $m_{H}$. Following the Higgs pair production, we address the couplings of the $C P$-even heavy Higgs to a pair of top quarks and to a pair of bottom quarks in sections 3 and 4 respectively. In section 5, we use the previous results to recast our limits in the purview of the phenomenological MSSM (pMSSM) and show the future reach of these searches in the $m_{A}-\tan \beta$ parameter space. We finally summarise our results and present our future outlook in section 6 . 


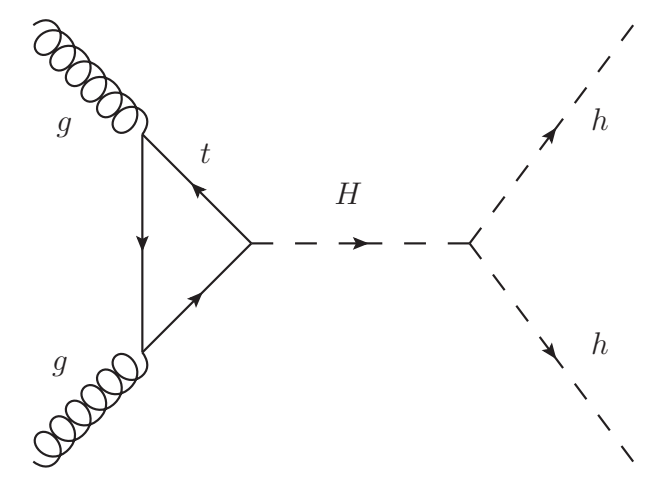

Figure 1. Feynman diagram for the signal production from $p p \rightarrow H \rightarrow h h$. The SM Higgses then decay to the corresponding final states viz. $h \rightarrow b \bar{b}$ and $h \rightarrow \gamma \gamma$ give rise to $b \bar{b} \gamma \gamma$ final state.

\section{$2 \quad$ The $p p \rightarrow H \rightarrow h h$ channel}

As discussed in the introduction, the objective of this work is to scrutinise the viable scalar extensions of the Standard Model (SM). In this section, we focus on a heavy $C P$-even scalar produced via gluon fusion and subsequently decaying to a pair of SM-like Higgs bosons. The decay width of the heavy Higgs boson is chosen to be $\sim 1 \mathrm{GeV}$ which is within the resolution of the detector. We would like to mention that the heavy Higgs search limits derived in the course of this analysis would stand valid only if the detector resolution is greater than the chosen heavy Higgs decay width. In the next five subsections, we study the reach of the HL-LHC in constraining the resonant Higgs pair production cross-section, $\sigma(p p \rightarrow H \rightarrow h h)$, upon studying multifarious channels, viz., $b \bar{b} \gamma \gamma, b \bar{b} b \bar{b}, b \bar{b} \tau^{+} \tau^{-}, b \bar{b} W^{+} W^{-}$ and $\gamma \gamma W^{+} W^{-}$. Many of these channels with $\tau$-leptons and $W$-bosons give different signatures upon considering leptonic or hadronic modes. We study all possible final states giving importance to the total rate as well the cleanliness. Unless otherwise stated, we generate the signal samples with Pythia 6 and for the background samples, we use MG5_aMC@NLO [143]. The showering and hadronisation is performed within the Pythia 6 [144] framework. The $b$-tagging efficiency and mistag efficiencies of a $c$-jet or a light jet posing as a $b$-tagged jet are employed as functions of the transverse momentum of the jet [145]. Unless explicitly mentioned, the CTEQ61 PDF set has been used throughout this work. Also, to take into account the detector effects, we use the fast-detector simulation package, Delphes-3.4.1 [146].

\subsection{The $b \bar{b} \gamma \gamma$ channel}

The $b \bar{b} \gamma \gamma$ final state is the golden channel when it comes to studying the non-resonant double Higgs production. The cleanliness of this channel, owing to smaller backgrounds, triumphs over the reduced rate $(B r(h \rightarrow \gamma \gamma) \sim 0.2 \%)$. Here however, we turn to a resonant scalar production which decays to a pair of SM-like Higgs bosons. Our goal is to ascertain the reach of the HL-LHC in measuring $\sigma(p p \rightarrow H \rightarrow h h)$ for a range of heavy Higgs masses $\left(m_{H}\right)$ (figure 1). One of the reasons for this final state being a favourite is that the reconstruction and identification precision of photons at the LHC is very high. 


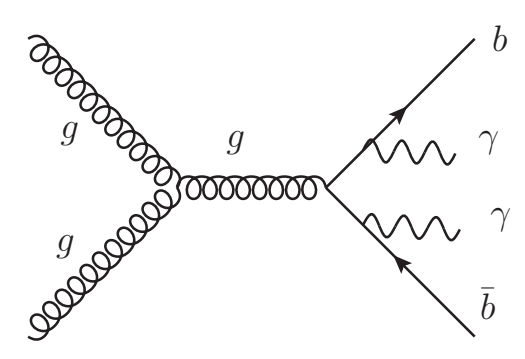

(a)

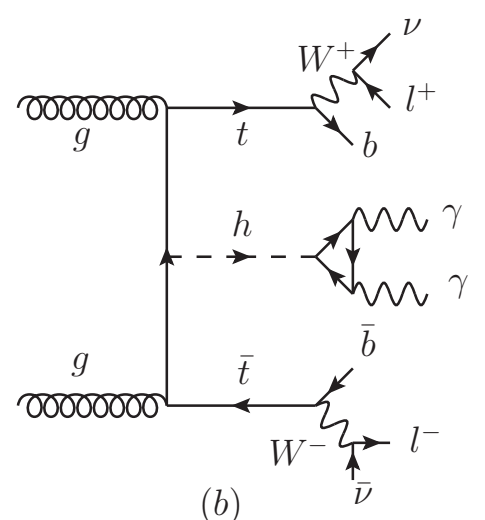

(b)

Figure 2. Feynman diagrams illustrating the (a) $b \bar{b} \gamma \gamma$ and (b) $t \bar{t} h$ background processes, corresponding to the $b \bar{b} \gamma \gamma$ search channel.

Even though the signal seems to have a clean final state, there are several backgrounds at play which need to be dealt with carefully. The major backgrounds (figure 2) typically have the form of $h h+X$ which includes the SM double Higgs production, $h+X$ which includes $Z h, h b \bar{b}$ and $t \bar{t} h$, and null-Higgs processes like $t \bar{t}+t \bar{t} \gamma$ where leptons may fake as photons, $b \bar{b} \gamma \gamma+c \bar{c} \gamma \gamma+j j \gamma \gamma$ (henceforth termed as $b \bar{b} \gamma \gamma^{*}$ ) where for the latter two, the light-jets may fake $b$-jets. Other fake backgrounds include $b \bar{b} j \gamma+c \bar{c} j \gamma$ (we will refer to it as Fake 1 category), $b \bar{b} j j$ (referred to as the Fake 2 category), where the $c$-jets may pose as $b$-jets and the light jet may mimic a photon, and the single Higgs processes, viz., $h j j+h c \bar{c}$ (classified henceforth as the $h j j^{*}$ category), where the light-jets and $c$-jets may mimic $b$-jets. One of the major differences between most of the backgrounds and the signal lies in the invariant mass distribution of the $b$-jets, $m_{b \bar{b}}$. However, even when the $m_{b \bar{b}}$ distribution of the signal (as well as the non-resonant SM di-Higgs background) peaks around the SM-like Higgs mass, $m_{h}$, it is broad and can have considerable overlap with the $m_{b \bar{b}}$ distribution either ensuing from a $Z$-boson or from a continuum. It should be noted that the most dominant backgrounds come from the QCD-QED $b \bar{b} \gamma \gamma^{*}, t \bar{t} h$ and SM-like di-Higgs processes. The former being a continuum, covers a large part of the kinematic variable space with the signal. The SM-like di-Higgs background also has very large overlap with the signal. One of the easiest way to break this degeneracy is to utilise the $m_{b \bar{b} \gamma \gamma}$ or reconstructed $m_{h h}$ distribution which has a clear peak around the heavy scalar mass, $m_{H}$, for the signal.

We generate the QCD-QED $b \bar{b} \gamma \gamma$ and $Z \gamma \gamma \rightarrow b \bar{b} \gamma \gamma$ backgrounds upon merging with one additional jet. We employ the MLM merging scheme [147] where the extra jet contains gluon, light quarks, $c$ - as well as $b$-quarks. Among the $h+X$ category, the $Z h$ is generated with the Higgs boson decaying to a pair of photons and the $Z$-boson decaying to a pair of bottom quarks. Furthermore, the $t \bar{t} h$ and $b \bar{b} h$ backgrounds are generated with $h \rightarrow \gamma \gamma$. The major fake backgrounds with jets in the final state are generated with the aforementioned jet definition with one exception. We define both of the jets in the $j j \gamma \gamma$ channel in a way as to have no overlap with the $b \bar{b} \gamma \gamma$ background. In case of the $t \bar{t}+X$ backgrounds, we generate 

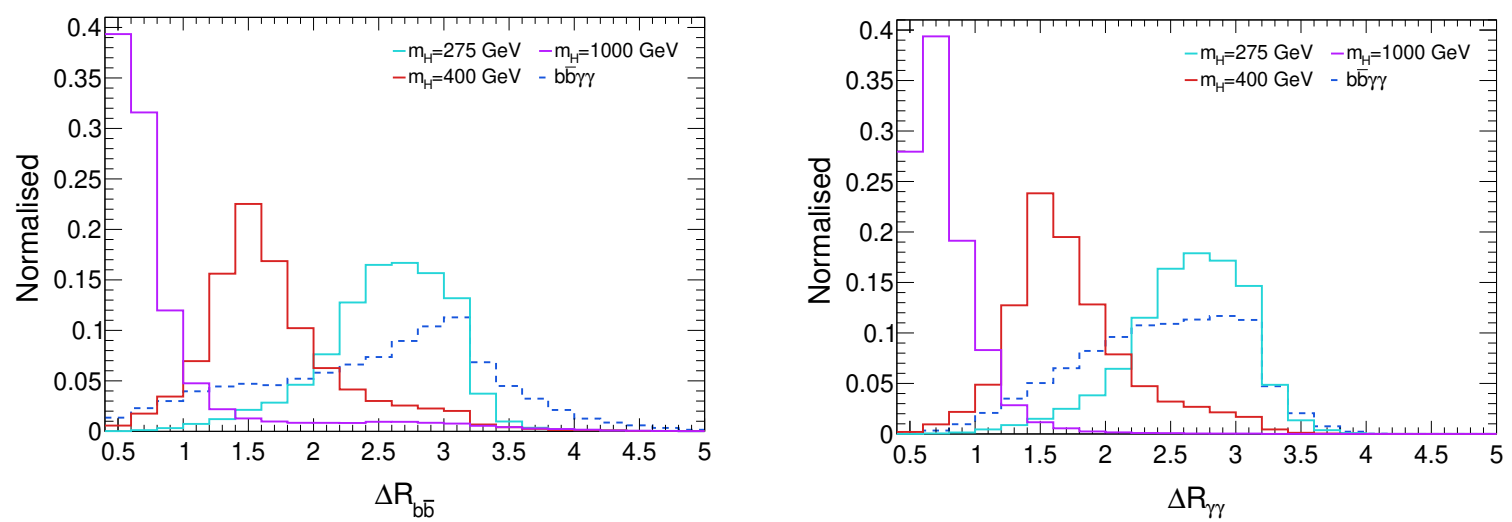

Figure 3. Normalised distributions of $\Delta R_{b b}$ and $\Delta R_{\gamma \gamma}$ for heavy Higgs masses, $m_{H}=275,400$ and $1000 \mathrm{GeV}$ with dominant $b \bar{b} \gamma \gamma$ background.

the $t \bar{t}$ events with both of the top quarks decaying leptonically which ultimately fakes as photons. However, for the $t \bar{t} \gamma$ background, we require one of the tops to decay leptonically and the other hadronically. Finally, we generate separate single Higgs backgrounds via gluon fusion in association with $c$-quarks and also with light jets. The separation between the $h c \bar{c}$ and $h j j$ backgrounds are necessary in order to appropriately take into account the different fake rates for $c \rightarrow b$ and $j \rightarrow b$. All of these backgrounds are generated with specific cuts at the generation level which we summarise in appendix A.

The idea of this section is to understand the reach of the HL-LHC in constraining models with extended scalar sectors. We thus employ optimised search strategies for a varied range of scalar masses. We vary $m_{H}$ in the mass range $275 \mathrm{GeV}$ and $1 \mathrm{TeV}$. Specifically, we consider the following benchmark points, viz., $m_{H}=275,300,350,400,450,500,550$, $600,800 \mathrm{GeV}$ and $1 \mathrm{TeV}$. In line with our previous work [105], we first perform a classical cut and count analysis to gauge the sensitivity of various benchmark points. We closely follow various cuts from the ATLAS projection study [61]. Namely, we require exactly two $b$-tagged jets and two photons in the final state. The photons are required to have transverse momenta, $p_{T}>10 \mathrm{GeV}$ and a pseudorapidity coverage of $|\eta|<2.5$. Moreover, the two photons are also required to lie within the pseudorapidity range, $\left|\eta_{\gamma}\right|<1.37$ (barrel region) or $1.52<\left|\eta_{\gamma}\right|<2.37$ (endcap region). After imposing these basic requirements, we apply some stronger selection cuts in order to enhance the signal to background ratio, $S / B$. We require the invariant mass of the pair of photons, $m_{\gamma \gamma}$, to reconstruct sharply about the SM-like Higgs mass in the range $(122,128) \mathrm{GeV}$. Furthermore, we veto events containing lepton(s) in the final state in order to reduce the impact of the $t \bar{t} h$ background when it decays semi-leptonically or leptonically. We also impose lower bounds on the transverse momenta of the leading and sub-leading $b$-jets and photons. Moreover, upon inspecting the distribution of $\Delta R_{\gamma \gamma}$ and $\Delta R_{b \bar{b}}$ (figure 3), we find that with larger values of $m_{H}$, the SM-like Higgs bosons are more boosted yielding more collimated final states. We thus require $\Delta R_{\gamma \gamma}$ and $\Delta R_{b \bar{b}}$ to lie in the range $(0.4,3.0),(0.4,2.0)$ and $(0.4,1.5)$ for $m_{H}=275,300$ and $350 \mathrm{GeV}, m_{H}=400,450,500,550$ and $600 \mathrm{GeV}$, and $m_{H}=800 \mathrm{GeV}$ and $1 \mathrm{TeV}$ respectively. Besides, we require the invariant mass of the $b$-jets, $m_{b \bar{b}}$ to lie in 


\begin{tabular}{c} 
Fixed cuts \\
\hline $122 \mathrm{GeV}<m_{\gamma \gamma}<128 \mathrm{GeV}$ \\
$N_{\ell}=0$ \\
$p_{T, b}>40(30) \mathrm{GeV}, p_{T, \gamma}>30(30) \mathrm{GeV}$ \\
$0.4<\Delta R_{\gamma \gamma}<(3.0 / 2.0 / 1.5), 0.4<\Delta R_{b b}<(3.0 / 2.0 / 1.5), \Delta R_{\gamma b}>0.4$ \\
$90 \mathrm{GeV}<m_{b b}<130 \mathrm{GeV}$ \\
\hline
\end{tabular}

Table 2. Applied fixed cuts for the cut-based analysis in the $b \bar{b} \gamma \gamma$ channel.

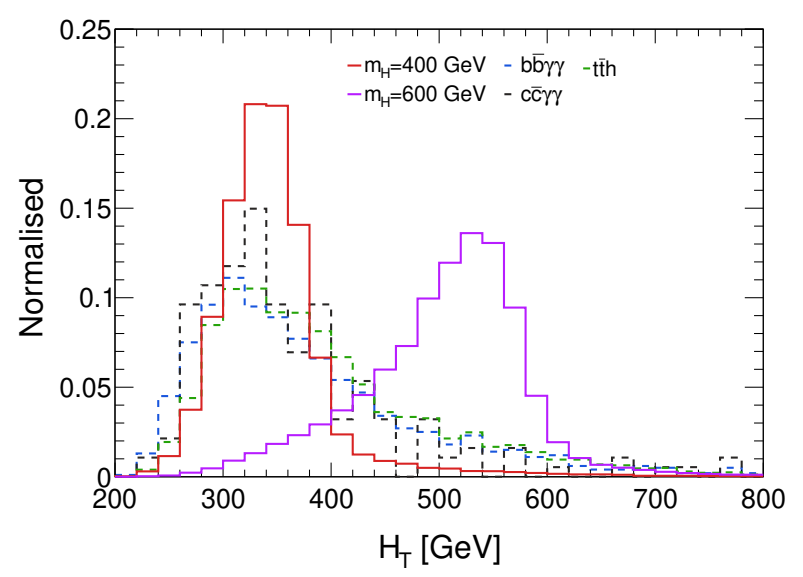

Figure 4. Normalised distribution of $H_{T}$ for heavy Higgs masses of $m_{H}=400,600 \mathrm{GeV}$ with dominant backgrounds.

the range $(90,130) \mathrm{GeV}$. This choice is related to account for the jet-energy correction and has been described in ref. [105]. We summarise these cuts in table 2. As the next logical step, we delve deeper into the kinematics. We reconstruct the invariant mass of the $b \bar{b} \gamma \gamma$ system, $m_{b \bar{b} \gamma \gamma}$ and its total visible energy, $H_{T}$. These two variables are intrinsically correlated. Also, from figure 4, it is evident that the $H_{T}$ distribution is broader leading to more background contamination as compared to the $m_{b \bar{b} \gamma \gamma}$ distribution which we show in figure 5 (left). Thus, we proceed with $m_{b \bar{b} \gamma \gamma}$ in order to further optimise our analysis. We also reconstruct the transverse momentum of the SM-like Higgs decaying to a pair of photons, $p_{T, \gamma \gamma}$. As can be seen from the $p_{T, \gamma \gamma}$ distribution in figure 5 , the spectrum is harder for heavier values of $m_{H}$. We choose $p_{T, \gamma \gamma}>50 \mathrm{GeV}$ for $m_{H}=275$ and $300 \mathrm{GeV}$ and for all other masses, we choose the transverse momentum of this reconstructed Higgs to be larger than $100 \mathrm{GeV}$. Thus, after these fixed cuts, we perform a simplified optimisation with the $m_{b \bar{b} \gamma \gamma}$ variable in order to enhance $S / B$. These cuts are finally tabulated in table 3 where we also present the signal efficiency and the background yield at an integrated luminosity, $\mathcal{L}=3 \mathrm{ab}^{-1}$ with $m_{H}$ being varied. The signal efficiency, $\epsilon$, here points to the ratio of the total number of signal events remaining after all the cuts applied in sequence to the generated number of events. The second column refers to the range of $m_{b \bar{b} \gamma \gamma}$ that optimises the signal and the third column denotes the minimum $p_{T}$ for the diphoton system. This 

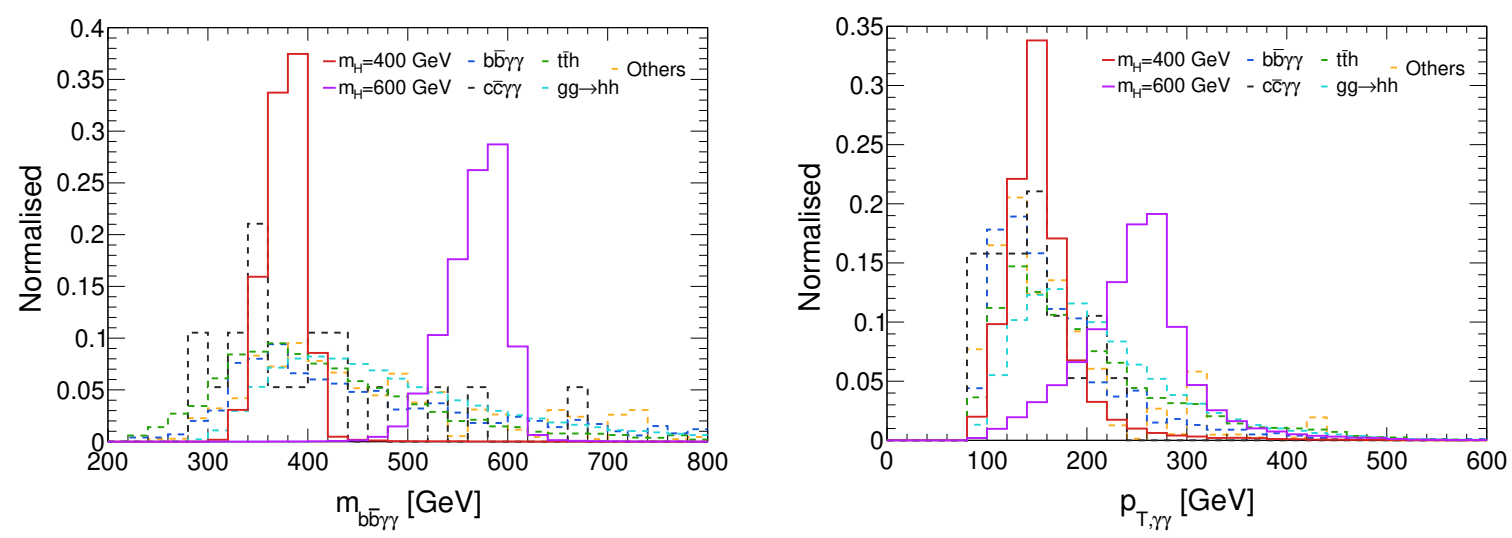

Figure 5. The $m_{b \bar{b} \gamma \gamma}$ and $p_{T, \gamma \gamma}$ distributions for heavy Higgs masses of $m_{H}=400$ and $600 \mathrm{GeV}$ with backgrounds. Here the heavy Higgs boson is searched for in the $b b \gamma \gamma$ final state. The distributions are shown after imposing the fixed cuts.

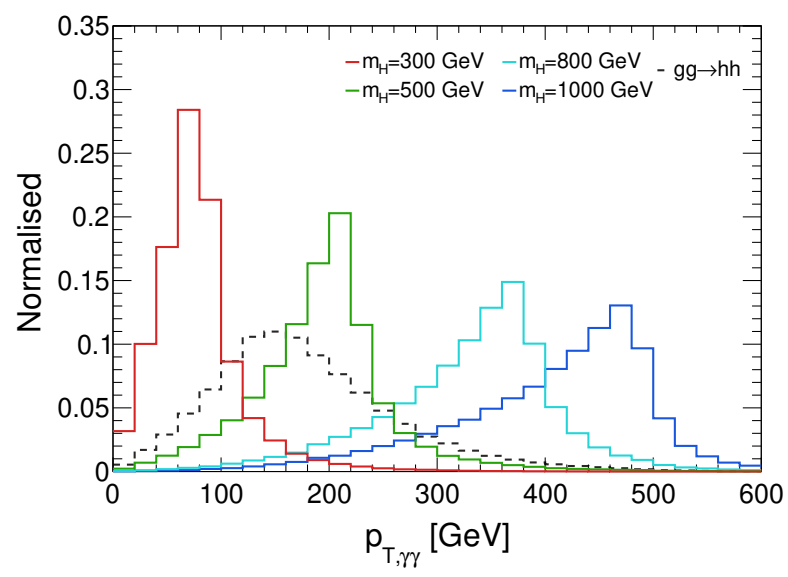

Figure 6. Normalised distribution of $p_{T, \gamma \gamma}$ for heavy Higgs masses of $m_{H}=300,500,800$ and $1000 \mathrm{GeV}$ along with the SM di-Higgs production from gluon-gluon fusion process.

optimisation is different as compared to the SM di-Higgs production scenario as shown in ref. [105]. This difference is related to the kinematics of the event topology. As an example, the $p_{T, \gamma \gamma}$ distribution changes with the heavy Higgs mass as shown in figure 6 . From this figure, it is evident that different optimisation is required for each mass point and also for the SM scenario. We must note that in table 3, the choices of the upper and lower cuts on $m_{b \bar{b} \gamma \gamma}$ for the different heavy Higgs masses can be understood from figure 7 . These ranges are obtained after optimising for each value of $m_{H}$. Finally, we provide a detailed cut-flow table for $m_{H}=400 \mathrm{GeV}$ in table 4 with a complete information for the backgrounds including the perturbative order at which the rates are considered as well as the number of events remaining at the HL-LHC.

Utilising these results, we derive the projected upper limits on the production cross section of the heavy Higgs in a model independent manner. ${ }^{1}$ We calculate the cross-section

\footnotetext{
${ }^{1}$ We consider the cut-based optimisations as final as we did not obtain any observable improvement with a multivariate analysis.
} 


\begin{tabular}{|c|c|c|c|c|}
\hline \multirow{2}{*}{$\begin{array}{c}\text { Heavy Higgs mass, } \\
m_{H}(\mathrm{GeV})\end{array}$} & \multicolumn{2}{|c|}{ Optimised cuts } & \multicolumn{2}{|c|}{ After all cuts } \\
\hline & $m_{b \bar{b} \gamma \gamma}(\mathrm{GeV})$ & $p_{T, \gamma \gamma}>(\mathrm{GeV})$ & Signal Efficiency $(\epsilon)$ & Background yield at $3000 \mathrm{fb}^{-1}$ \\
\hline 275 & {$[235,275]$} & 50 & 0.012 & 30.01 \\
\hline 300 & {$[255,305]$} & 50 & 0.024 & 55.62 \\
\hline 350 & {$[300,355]$} & 100 & 0.024 & 23.33 \\
\hline 400 & {$[345,405]$} & 100 & 0.032 & 15.80 \\
\hline 450 & {$[395,455]$} & 100 & 0.042 & 13.75 \\
\hline 500 & {$[445,510]$} & 100 & 0.051 & 10.87 \\
\hline 550 & {$[460,570]$} & 100 & 0.068 & 14.39 \\
\hline 600 & {$[460,615]$} & 100 & 0.076 & 18.11 \\
\hline 800 & {$[560,830]$} & 100 & 0.091 & 9.54 \\
\hline 1000 & {$[780,1030]$} & 100 & 0.090 & 2.31 \\
\hline
\end{tabular}

Table 3. Details of the final optimised cuts with signal efficiency and background yields after applying all cuts.

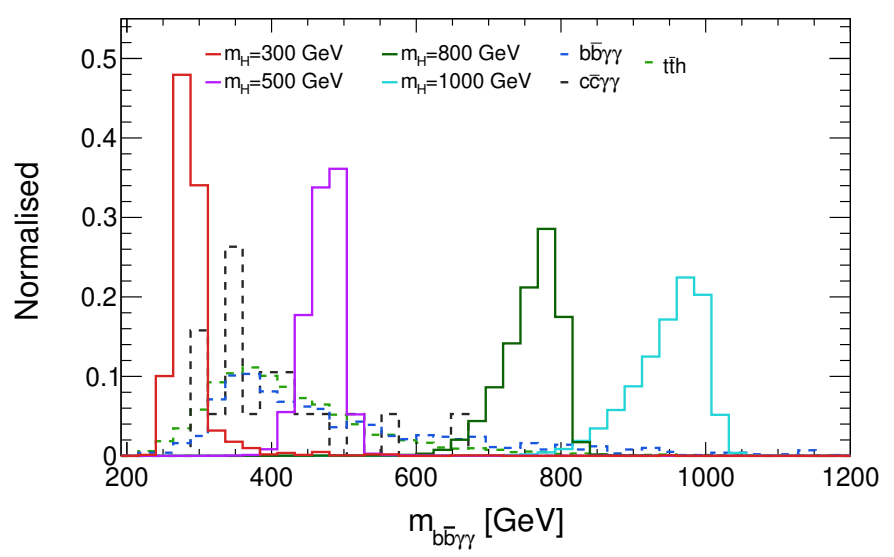

Figure 7. Normalised distribution of $m_{b \bar{b} \gamma \gamma}$ for heavy Higgs masses of $m_{H}=300,500,800$ and $1000 \mathrm{GeV}$ with dominant backgrounds.

reach by using the significance formula: $S / \sqrt{B}=N$, where $N$ denotes the number of confidence intervals. Here, the signal yield, $S$, is defined as $\sigma(p p \rightarrow H \rightarrow h h \rightarrow b \bar{b} \gamma \gamma) \times \mathcal{L} \times \epsilon$ and $B$ represents the total background yield after the cut-based analysis. With this, we derive $\sigma(p p \rightarrow H \rightarrow h h)$ at the $N \sigma$ level, with $N=2$ and 5 , respectively, corresponding to a $95 \%$ and $99.7 \%$ confidence level (CL) upper limit, also referred to as the exclusion and discovery limits, respectively. We show the final results in figure 8 with the upper limit on $\sigma(p p \rightarrow H \rightarrow h h)$ as a function of $m_{H}$ and we display the $2 \sigma$ and $5 \sigma$ lines. The $2 \sigma$ upper limit is strong between $400 \mathrm{GeV}$ and $1 \mathrm{TeV}$, varying between $31.74 \mathrm{fb}$ and $4.24 \mathrm{fb}$. Upon adding $5 \%$ systematic uncertainty, ${ }^{2}$ the upper limit becomes $32.35 \mathrm{fb}$ and $4.25 \mathrm{fb}$ respectively within the previously mentioned mass range. It must be noted that the upper limit is mildly affected by incorporating a systematic uncertainty of $\sim 5 \%$. The reason can be attributed to the fact that the signal over background ratio $(S / B)$ is high.

\footnotetext{
${ }^{2}$ The significance formula for a systematic uncertainty of $N \%$ has the form: $S / \sqrt{B+(N * B / 100)^{2}}$.
} 


\begin{tabular}{|c|c|c|c|c|c|c|c|c|c|c|}
\hline \multirow{3}{*}{ Cut flow } & \multirow{3}{*}{$\begin{array}{c}\text { Signal Efficiency } \\
\quad p p \rightarrow H \\
\rightarrow h h \rightarrow 2 b 2 \gamma)\end{array}$} & \multicolumn{9}{|c|}{ Event rates with $\mathcal{L}=3 \mathrm{ab}^{-1}$} \\
\hline & & \multicolumn{9}{|c|}{ SM Backgrounds } \\
\hline & & $h h \rightarrow 2 b 2 \gamma$ & $h b \bar{b}$ & $t \bar{t} h$ & $Z h$ & $Z \gamma \gamma$ & $b \bar{b} \gamma \gamma *^{a}$ & Fake $1^{b}$ & Fake $2^{c}$ & $h j j^{* d}$ \\
\hline Order & & NNLO [148] & $\begin{array}{c}\text { NNLO }(5 \mathrm{FS})+ \\
\mathrm{NLO}(4 \mathrm{FS})[149]\end{array}$ & NLO [149] & $\begin{array}{c}\text { NNLO (QCD) + } \\
\text { NLO EW [149] }\end{array}$ & LO & $\mathrm{LO}$ & LO & LO & LO \\
\hline$m_{\gamma \gamma}$ & 0.123 & 39.71 & 36.68 & 397.97 & 62.21 & 32.86 & 1071.38 & 837.45 & 403.98 & 9.60 \\
\hline$N_{\ell}$ & 0.122 & 39.70 & 36.68 & 290.10 & 62.21 & 32.86 & 1071.34 & 837.40 & 403.98 & 9.60 \\
\hline$p_{T, b / \gamma}$ & 0.081 & 27.65 & 16.34 & 197.83 & 35.87 & 14.00 & 510.73 & 361.01 & 183.70 & 4.91 \\
\hline$\Delta R$ cuts & 0.052 & 20.56 & 5.09 & 36.73 & 22.32 & 4.86 & 56.24 & 35.60 & 27.05 & 1.53 \\
\hline$m_{b b}$ & 0.036 & 14.19 & 1.41 & 12.74 & 4.43 & 1.02 & 16.44 & 11.47 & 7.47 & 0.41 \\
\hline$p_{T, \gamma \gamma}$ & 0.035 & 14.01 & 1.36 & 12.29 & 4.28 & 0.98 & 15.53 & 10.90 & 6.70 & 0.40 \\
\hline$m_{b \bar{b} \gamma \gamma}$ & 0.032 & 2.96 & 0.29 & 3.31 & 0.87 & 0.21 & 3.84 & 3.18 & 1.03 & 0.08 \\
\hline
\end{tabular}

${ }^{a} b \bar{b} \gamma \gamma+c \bar{c} \gamma \gamma+j j \gamma \gamma$.

${ }^{b} b \bar{b} j \gamma+c \bar{c} j \gamma$.

${ }^{c} b \bar{b} j j$.

${ }^{d}(g g \rightarrow h j j)+(g g \rightarrow h c \bar{c})$.

Table 4. The cut-flow table for heavy Higgs of mass $400 \mathrm{GeV}$. The table also shows the various perturbative orders at which the cross-sections have been used.

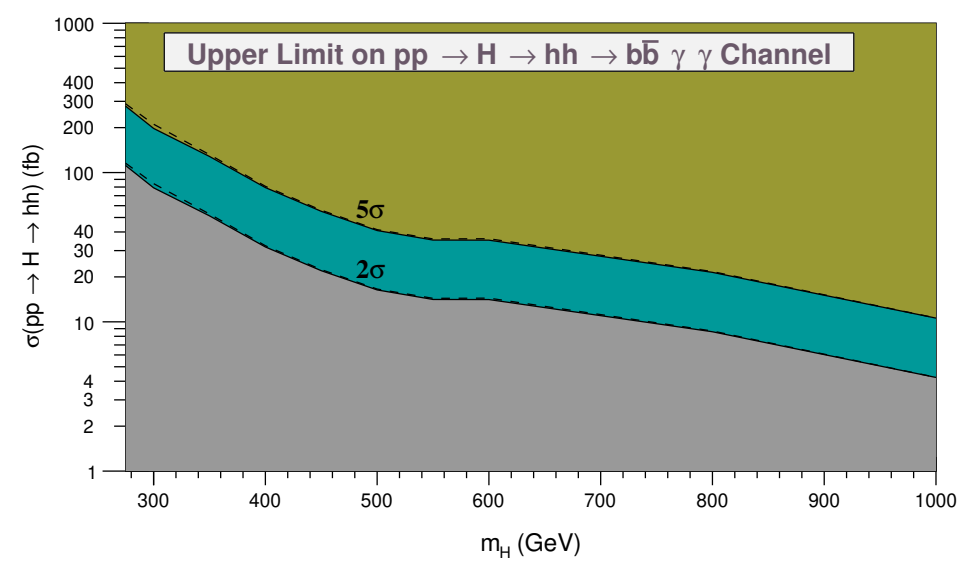

Figure 8. Upper limit on $\sigma(p p \rightarrow H \rightarrow h h)(\mathrm{fb})$ as a function of $m_{H}(\mathrm{GeV})$ for the $b \bar{b} \gamma \gamma$ channel with cut-based analysis. The solid (dashed) lines show the $2 \sigma-5 \sigma$ band on taking $0 \%$ ( $5 \%$ ) systematic uncertainties.

Next, we perform a multivariate analysis in order to improve upon the cut-based analysis. We use the following variables:

$$
\begin{aligned}
& m_{b b}, \Delta R_{b b}, p_{T, \gamma \gamma}, \Delta R_{\gamma \gamma}, m_{b \bar{b} \gamma \gamma}, \Delta R_{b_{i} \gamma_{j}} \\
& \Delta R_{b b, \gamma \gamma}, p_{T, b_{1}}, p_{T, b_{2}}, p_{T, \gamma_{1}}, p_{T, \gamma_{2}}
\end{aligned}
$$

Here, the variable names have their usual meaning. The $\Delta R_{b_{i} \gamma_{j}}$ is the distance in the $\eta-\phi$ plane between the $b$-jets and photons with $i$ and $j=1,2$. Also, $\Delta R_{b b, \gamma \gamma}$ is the $\Delta R$ separation between the system of $b$-jets and the two photon system. However, after performing this analysis we obtain comparable results. Thus, we do not show the results of the multivariate analysis in this section. 


\begin{tabular}{c}
\hline Fixed cuts \\
\hline $122 \mathrm{GeV}<m_{\gamma \gamma}<128 \mathrm{GeV}$ \\
$p_{T, b}>35(25) \mathrm{GeV}, p_{T, \gamma}>30(30) \mathrm{GeV}$ \\
$0.4<\Delta R_{\gamma \gamma}<(3.0 / 2.0 / 1.5), 0.4<\Delta R_{b b}<(3.0 / 2.0 / 1.5), \Delta R_{\gamma b}>0.4$ \\
$55 \mathrm{GeV}<m_{b b}<100 \mathrm{GeV}$ \\
\hline
\end{tabular}

Table 5. Applied fixed cuts for the cut-based analysis.

\subsubsection{The $p p \rightarrow A \rightarrow Z h$ channel}

With the accumulation of more data, we are on the brink of accepting the fact that in MSSM or in generic two Higgs doublet models, the SM-like Higgs is in the decoupling regime with its coupling to the SM gauge bosons being proportional to $\sin (\beta-\alpha)$, with $\alpha$ and $\tan \beta$ being the mixing angle in the neutral $C P$-even sector and the ratio of the two vacuum expectation values of the two doublets, respectively. For the up and down type fermions, the Yukawa couplings for the SM-like Higgs boson are proportional to $\cos \alpha / \sin \beta$ and $\sin \alpha / \cos \beta$ respectively. In the decoupling regime, $\sin (\beta-\alpha) \sim 1$ and hence the decay width of $A \rightarrow Z h$ which is proportional to the coupling $\cos (\beta-\alpha)$, is small. In a nondecoupling regime, $p p \rightarrow A \rightarrow Z h$ can give us deep insight into two scalars simultaneously.

The CMS [150] collaboration has derived projected upper limits on $\sigma(p p \rightarrow A \rightarrow Z h)$ from searches in the $\ell \ell b \bar{b}(Z \rightarrow \ell \ell, h \rightarrow b \bar{b})$ final state for HL-LHC. In the present study, keeping in continuation to the analysis prescribed in the previous section (section 2.1), we explore the prospects of directly probing $A$ in the $A \rightarrow Z h \rightarrow b \bar{b} \gamma \gamma$ channel in the context of HL-LHC.

We will remain agnostic to the fact that the prospects of observing $A \rightarrow Z h$ in the decoupling regime may be extremely small. The main difference here is the fact that in the previous analysis both the diphoton and the $b \bar{b}$ pairs are required to peak around the SM-like Higgs boson mass, whereas in the present case, the $b$-jets are required to peak around the $Z$-boson mass. We follow a cut-based analysis as before and optimise the $m_{b \bar{b} \gamma \gamma}$ and $p_{T, \gamma \gamma}$ cuts for different values of $m_{A}$. These variables are shown to have substantial discriminatory power and are shown in figure 9 for $m_{A}=400 \mathrm{GeV}$ and $600 \mathrm{GeV}$. Details of the fixed and optimised cuts are presented in table 5 and table 6 respectively. In table 7 , we show the cut-flow table for $m_{A}=400 \mathrm{GeV}$. After a full optimisation, we show the $95 \%$ and $99.7 \%$ CL exclusion for $\sigma(p p \rightarrow A \rightarrow Z h)$ in figure 10. The bounds are weaker than their $H \rightarrow h h$ counterpart mainly because of a larger overlap with the $Z h$ background. It is to be noted that the projected upper limits on $\sigma(p p \rightarrow A \rightarrow Z h)$ derived from searches in the $b \bar{b} \gamma \gamma$ channel (see figure 10) are weaker than the projected limit obtained by CMS from searches in the $\ell \ell b \bar{b}[150]$ final state.

\subsection{The $b \bar{b} b \bar{b}$ channel}

After having studied the cleanest possible di-Higgs final state, we turn our attention to the one with the largest rate, viz., $p p \rightarrow H \rightarrow h h \rightarrow 4 b$. Several searches have already been conducted in this channel $[58,134,135,151]$ and provide some of the strongest bounds 

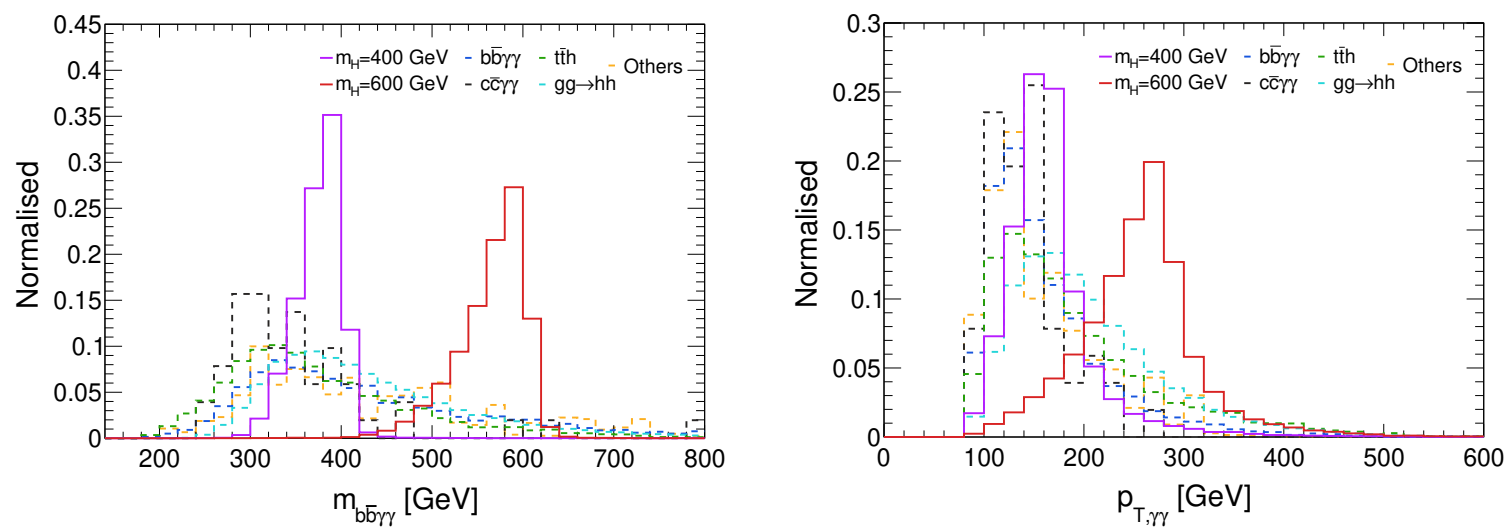

Figure 9. The $m_{b \bar{b} \gamma \gamma}$ and $p_{T, \gamma \gamma}$ distributions for heavy Higgs masses of $m_{A}=400$ and $600 \mathrm{GeV}$ with backgrounds. Here the heavy pseudoscalar is searched for in the $b b \gamma \gamma$ final state. The distributions are shown after imposing the fixed cuts.

\begin{tabular}{|c|c|c|c|c|}
\hline \multirow{2}{*}{$\begin{array}{l}\text { Heavy Pseudoscalar mass, } \\
\qquad m_{A}(\mathrm{GeV})\end{array}$} & \multicolumn{2}{|c|}{ Optimised cuts } & \multicolumn{2}{|c|}{ After all cuts } \\
\hline & $m_{b \bar{b} \gamma \gamma}(\mathrm{GeV})$ & $p_{T, \gamma \gamma}>(\mathrm{GeV})$ & Signal Efficiency $\left(\times 10^{-2}\right)$ & Background yield at $3000 \mathrm{fb}^{-1}$ \\
\hline 220 & {$[170,235]$} & 50 & 0.48 & 30.40 \\
\hline 300 & {$[255,305]$} & 50 & 3.24 & 91.87 \\
\hline 350 & {$[290,360]$} & 100 & 4.01 & 51.08 \\
\hline 400 & {$[345,420]$} & 100 & 5.00 & 34.10 \\
\hline 600 & {$[470,625]$} & 100 & 10.19 & 24.28 \\
\hline 800 & {$[590,830]$} & 100 & 10.68 & 10.69 \\
\hline 1000 & {$[780,1040]$} & 100 & 7.50 & 4.15 \\
\hline
\end{tabular}

Table 6. Details of the final optimised cuts with signal efficiency and background yields after applying all cuts.

\begin{tabular}{|c|c|c|c|c|c|c|c|c|c|c|}
\hline \multirow{3}{*}{ Cut flow } & \multirow{3}{*}{$\begin{array}{c}\text { Signal Efficiency } \\
\text { for } p p \rightarrow A \rightarrow Z h \\
\quad \rightarrow 2 b 2 \gamma\end{array}$} & \multicolumn{9}{|c|}{ Event rates with $3000 \mathrm{fb}^{-1}$ of integrated luminosity } \\
\hline & & \multicolumn{9}{|c|}{ SM Backgrounds } \\
\hline & & $h h \rightarrow 2 b 2 \gamma$ & $h b \bar{b}$ & $t \bar{t} h$ & $Z h$ & $Z \gamma \gamma$ & $b \bar{b} \gamma \gamma *^{a}$ & Fake $1^{b}$ & Fake $2^{c}$ & $h j j^{* d}$ \\
\hline$m_{\gamma \gamma}$ & 0.115 & 39.71 & 36.68 & 397.97 & 62.21 & 32.86 & 1071.38 & 837.45 & 403.98 & 9.60 \\
\hline$p_{T, b / \gamma}$ & 0.091 & & 26.29 & 314.39 & 47.00 & 19.82 & 670.56 & 483.95 & 241.41 & 7.12 \\
\hline$\Delta R$ cuts & & 88 & 8.28 & 62.03 & 25.88 & 5.86 & 7 & 48.40 & 27 & 2.32 \\
\hline$m$ & 0.059 & 9.02 & 3.03 & 23.85 & 22.56 & 5.03 & 35.40 & 21.94 & 5.72 & 0.88 \\
\hline$p_{T, \gamma \gamma}$ & 058 & 8.89 & 2.86 & 22.77 & 21.50 & 4.80 & 31.30 & 20.48 & 13.91 & 0.84 \\
\hline$m_{b \bar{b} \gamma \gamma}$ & 0.050 & 2.94 & \begin{tabular}{|l|}
0.64 \\
\end{tabular} & 6.39 & 5.97 & 1.28 & 8.16 & 5.68 & 2.83 & 0.21 \\
\hline
\end{tabular}

${ }^{a} b \bar{b} \gamma \gamma+c \bar{c} \gamma \gamma+j j \gamma \gamma$

${ }^{b} b \bar{b} j \gamma+c \bar{c} j \gamma$.

${ }^{c} b \bar{b} j j$.

${ }^{d}(g g \rightarrow h j j)+(g g \rightarrow h c \bar{c})$.

Table 7. The cut-flow table for a pseudoscalar mass of $400 \mathrm{GeV}$. The various perturbative orders used in the calculations are the same as in table 4 . 


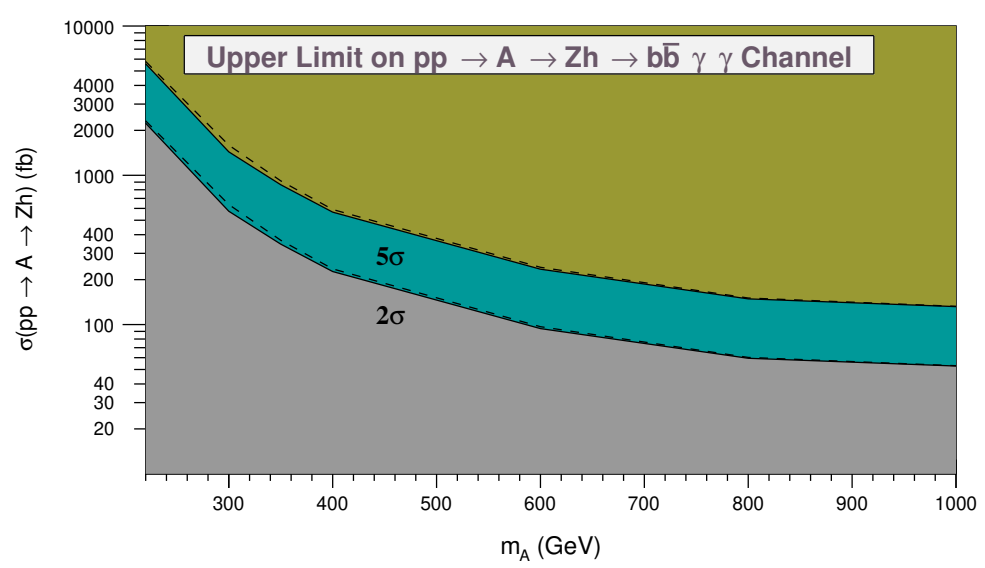

Figure 10. Upper limit on $\sigma(p p \rightarrow A \rightarrow Z h)(\mathrm{fb})$ as a function of $m_{A}(\mathrm{GeV})$ for the $b \bar{b} \gamma \gamma$ channel with cut-based analysis. The solid (dashed) lines show the $2 \sigma-5 \sigma$ band on taking $0 \%(5 \%)$ systematic uncertainties.

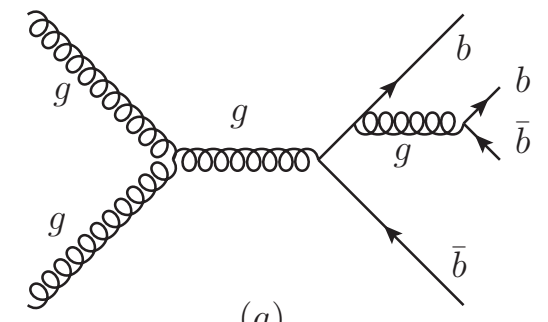

$(a)$

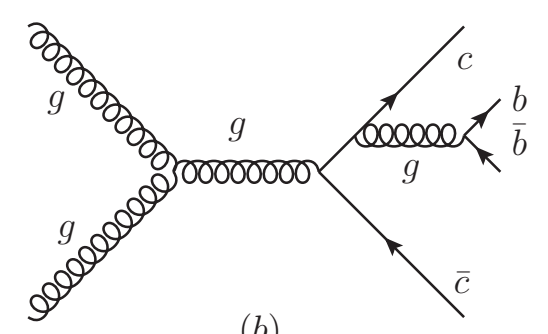

(b)

Figure 11. Feynman diagrams for dominant (a) $b \bar{b} b \bar{b}$ and (b) $b \bar{b} c \bar{c}$ background for the $b \bar{b} b \bar{b}$ channel.

in both the non-resonant and resonant sectors. ATLAS [135] has computed the observed (expected) upper bound on $\sigma(p p \rightarrow h h \rightarrow 4 b)$ to be 13 (20.7) times that of the SM expectation with an integrated luminosity of $27.5 \mathrm{fb}^{-1}$. This channel has been further combined in ATLAS' HL-LHC projection [152] alongside $p p \rightarrow h h \rightarrow b \bar{b} \gamma \gamma / b \bar{b} \tau \tau$. The above channel will gain an improvement in sensitivity according to the ref. [152] because of a projected $8 \%$ improvement in $b$-tagging efficiency, besides having larger data sets. In this work, we closely follow the search strategy used by the ATLAS collaboration in ref. [58]. Even though this channel has the highest signal rate, the enormous multijet and $t \bar{t}$ backgrounds may considerably overwhelm the signal yield.

The dominant backgrounds (figure 11) include the multijet production from QCD processes and the top pair production. For the multijet backgrounds, we dissect our sample generation into three different categories each having at least two $b$-quarks, viz., $b \bar{b} b \bar{b}, b \bar{b} c \bar{c}$ and $b \bar{b} j j$, in order to have sufficient statistics to take into account the different tagging efficiencies and fake rates. We do not generate the $h+$ jets and $Z+$ jets backgrounds separately but we include their tree-level diagrams while generating the multijet backgrounds as they have negligible contributions [58]. We must mention here that we do not consider other possible sources of multijet production viz., $c \bar{c} c \bar{c}, c \bar{c} j j$ etc. as these processes will be highly suppressed (with respect to $b \bar{b} b \bar{b}$ ) upon multiplying by the fake efficiency factors, in 


\begin{tabular}{|c|c|c|c|c|c|c|c|c|c|c|}
\hline \multirow{4}{*}{ Cut flow } & \multirow{2}{*}{\multicolumn{4}{|c|}{$\begin{array}{l}\text { Signal Efficiency }\left(\times 10^{-3}\right) \\
\quad(p p \rightarrow H \rightarrow h h \rightarrow b \bar{b} b \bar{b})\end{array}$}} & \multicolumn{6}{|c|}{ Cross section [fb] } \\
\hline & & & & & \multicolumn{6}{|c|}{ SM Backgrounds } \\
\hline & \multicolumn{4}{|c|}{ For $m_{H}$ of } & \multirow[t]{2}{*}{$b \bar{b} b \bar{b}$} & \multirow[t]{2}{*}{$b \bar{b} c \bar{c}$} & \multirow[t]{2}{*}{$t \bar{t}$} & \multirow[t]{2}{*}{$b \bar{b} j j$} & \multirow[t]{2}{*}{$h h \rightarrow 4 b$} & \multirow[t]{2}{*}{$t \bar{t} b \bar{b}$} \\
\hline & $400 \mathrm{GeV}$ & $600 \mathrm{GeV}$ & $800 \mathrm{GeV}$ & $1000 \mathrm{GeV}$ & & & & & & \\
\hline Order & - & - & - & - & $\mathrm{LO}$ & $\mathrm{LO}$ & NNLO + NNLL [153] & LO & NNLO [148] & $\mathrm{LO}$ \\
\hline $4 b$-jets & 5.40 & 30.79 & 56.36 & 67.29 & 278.15 & 26.85 & 2.83 & 66.74 & 0.16 & 8.29 \\
\hline$H_{T}$ & 5.05 & 30.69 & 56.32 & 67.26 & 263.80 & 25.48 & 2.78 & 64.15 & 0.16 & 8.27 \\
\hline$\Delta R_{b b}$ & 1.67 & 24.80 & 48.00 & 57.34 & 93.67 & 7.94 & 1.72 & 11.90 & 0.12 & 1.87 \\
\hline$p_{T, \text { di-jet }}$ & 0.41 & 21.19 & 46.44 & 56.52 & 54.25 & 4.69 & 1.28 & 7.54 & 0.09 & 1.61 \\
\hline$m_{t}$ & 0.33 & 18.25 & 38.34 & 45.83 & 46.43 & 3.80 & 0.20 & 6.36 & 0.08 & 0.66 \\
\hline
\end{tabular}

Table 8. Cut-flow table before performing the multivariate analysis, in the $b \bar{b} b \bar{b}$ channel.

succession. We generate the $t \bar{t}$ background with the top quark decaying to a $b$-quark and a $W$-boson. The $W$-bosons are then further decayed to $c \bar{s}$ or $\bar{c} s$. We avoid the $W \rightarrow u \bar{d}$ mode as the probability of a light jet faking a $b$-jet is $\sim 10$ times smaller than that of a $c$-jet. Lastly, we also consider the subdominant background coming from the non-resonant di-Higgs production $(g g \rightarrow h h)$ and also from $t \bar{t} b \bar{b}$ (including $t \bar{t} Z / t \bar{t} h$ ).

We select events containing exactly $4 b$-tagged jets with the requirement of $p_{T, b}>60 \mathrm{GeV}$ and $\left|\eta_{b}\right|<2.5$. The scalar sum, $H_{T}$, of the transverse momenta of all the visible particles in an event must fulfil, $H_{T}>300 \mathrm{GeV}$. Finally, we form two di-jet systems from these four $b$-jets. The two jets within a dijet system must satisfy $0.4<\Delta R_{b b}<1.5$. We choose the leading (sub-leading) di-jet system to have $p_{T}>200$ (150) GeV. ${ }^{3}$ Furthermore, to reduce the contamination from the $t \bar{t}$ background, we reconstruct the top by combining extra jets in an event with the di-jet systems. These jets must be within $\Delta R<1.5$ in the $\eta-\phi$ plane with the di-jet system. If an event contains exactly one extra jet, then we choose the di-jet system which is closest to it and combine to form a top quark system, $m_{t_{1}}$. However, when there are two such jets, we compute the minimum of all possible $\Delta R$ combinations between these two jets and the two di-jets before reconstructing two other top masses, $m_{t_{2}}$ and $m_{t_{3}}$. Because for our signal, we do not expect any proper top quark reconstruction, we thus veto events if the reconstructed mass of any of these possible choices for the top quark exceeds $120 \mathrm{GeV}$. After imposing this cut the $t \bar{t}$ background reduces to half with more than $80 \%$ of the signal events still to spare. We detail these cuts one by one alongside the signal efficiency and cross-sections for the background processes in table 8 .

Finally, after all the aforementioned cuts are applied in succession, we check for any possible improvement upon performing a multivariate analysis. We utilise the BDT algorithm for our purposes and choose the following nine kinematic variables with maximal

\footnotetext{
${ }^{3}$ These are preliminary cuts before performing the multivariate analysis. The rationale behind these cuts are (a) some of these cuts have been applied at the generation level on some of the backgrounds in order to have better control over event statistics owing to large production cross-sections (see appendix A) and (b) some other cuts are applied by observing the kinematic distributions of these observables. However, we apply stronger cuts on these variables in the following where we optimise them alongside other correlated variables through a more sophisticated multivariate analysis.
} 

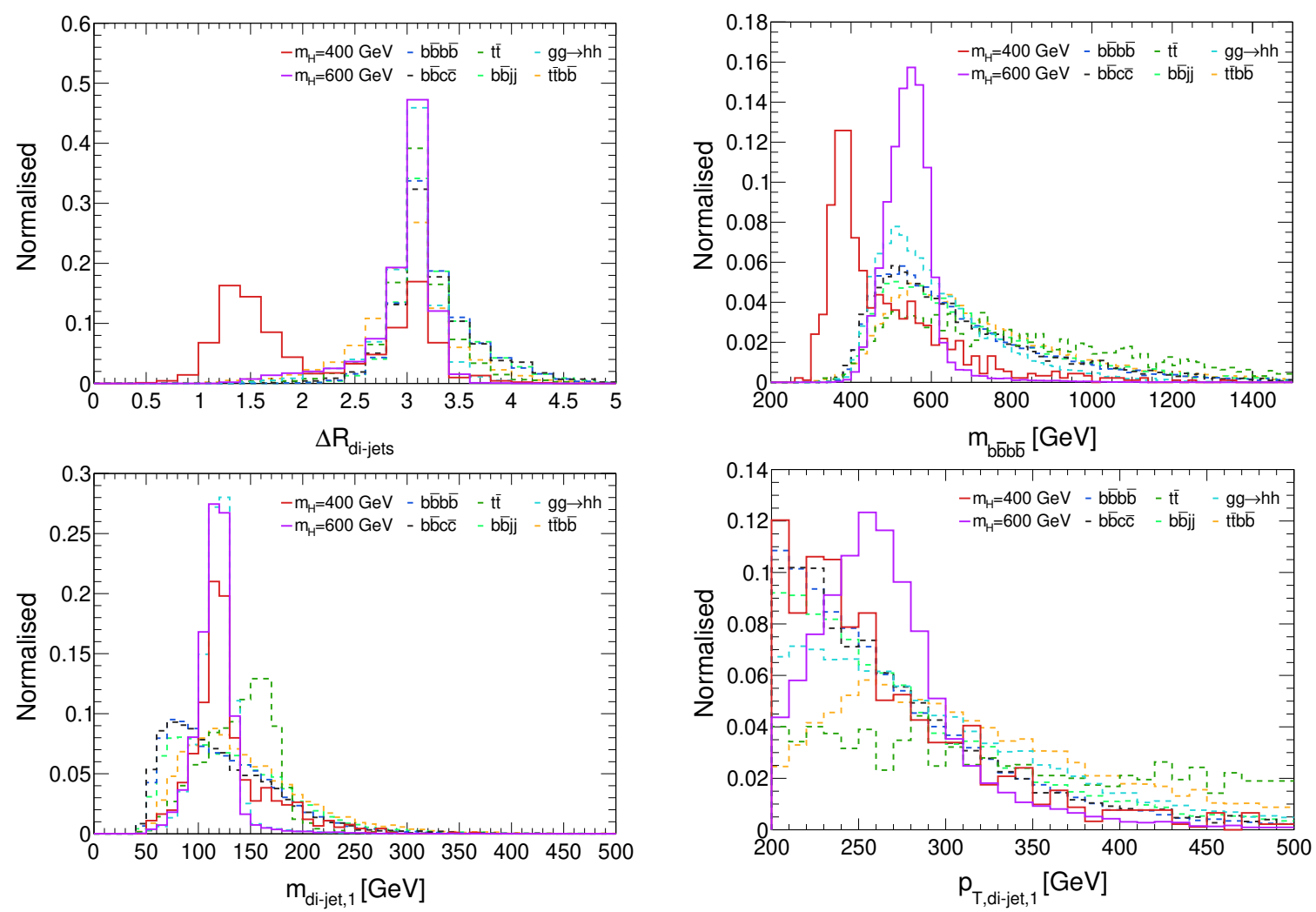

Figure 12. The $\Delta R_{\text {di-jets }}, m_{4 b}, m_{\text {di-jet }, 1}$ and $p_{T, \text { di-jet }, 1}$ distributions for heavy Higgs masses of $m_{H}=400$ and $600 \mathrm{GeV}$ with backgrounds. Here the heavy Higgs boson is searched for in the $4 b$ final state. The distributions are shown after imposing the cuts mentioned in table 8 .

potency,

$$
p_{T, \text { di-jet }, k}, m_{\text {di-jet }, k}, \Delta \phi_{b b, \text { di-jet }, k}, \Delta \eta_{\text {di-jets }}, \Delta R_{\text {di-jets }}, m_{b \bar{b} b \bar{b}}
$$

Here we use the kinematic variables reconstructed from the two di-jet systems viz., invariant mass $\left(m_{\text {di-jet }}\right)$, transverse momentum $\left(p_{T, \text { di-jet }}\right)$ and azimuthal angle separation between the $b$-jets forming the dijet systems $\left(\Delta \phi_{b b \text {,di-jet }}\right)$. The subscript $k=1,2$ refers to the $p_{T}$ ordering of the di-jets. We also take the separation in the $\eta$ and $\eta-\phi$ plane between the two di-jets, viz., $\Delta \eta_{\text {di-jets }}$ and $\Delta R_{\text {di-jets }}$ respectively. $m_{b \bar{b} b \bar{b}}$ is the invariant mass of the four $b$-jet system. The top four variables with the highest discriminatory power are shown in figure 12 . We can see that the lower masses have significantly longer tails while performing the mass reconstructions.

Finally, in table 9, we present the background yields after the BDT optimisation has been completed. Like in the previous section, we translate these results into an exclusion diagram showing the upper limits on $\sigma(p p \rightarrow H \rightarrow h h)$ as a function of the heavy Higgs mass. We show these in figure 13. The limit is very strong between $600 \mathrm{GeV}$ and $1 \mathrm{TeV}$ with the $95 \%$ CL upper limit varying between $15.26 \mathrm{fb}$ and $2.51 \mathrm{fb}$. The upper limit becomes between $[82.70,5.77] \mathrm{fb}$ within the aforementioned range by adding $5 \%$ systematic uncertainty,. 


\begin{tabular}{|c|c|c|c|}
\hline (a) & Process & Order & Events \\
\hline \multirow{7}{*}{ Background } & $b \bar{b} b \bar{b}$ & LO & 203.60 \\
\hline & $b \bar{b} c \bar{c}$ & LO & 121.51 \\
\hline & $b \bar{b} j j$ & LO & 46.11 \\
\hline & $t \bar{t}$ & NNLO [153] & 10.65 \\
\hline & $p p \rightarrow h h$ & NNLO [148] & 6.77 \\
\hline & $t \bar{t} b \bar{b}$ & $\mathrm{LO}$ & 77.28 \\
\hline & \multicolumn{2}{|c|}{ Total } & 465.92 \\
\hline
\end{tabular}

\begin{tabular}{|c|c|c|}
\hline (b) & Process & Events \\
\hline & $b \bar{b} b \bar{b}$ & 8950.94 \\
& $b \bar{b} c \bar{c}$ & 731.91 \\
& $b \bar{b} j j$ & 1263.16 \\
Background & $t \bar{t}$ & 74.56 \\
& $p p \rightarrow h h$ & 103.65 \\
& $t \bar{t} b \bar{b}$ & 230.05 \\
\cline { 2 - 3 } & Total & 11354.27 \\
\hline
\end{tabular}

\begin{tabular}{|c|c|c|}
\hline$(\mathrm{c})$ & Process & Events \\
\hline & $b \bar{b} b \bar{b}$ & 4304.59 \\
& $b \bar{b} c \bar{c}$ & 358.89 \\
& $b \bar{b} j j$ & 624.17 \\
Background & $t \bar{t}$ & 130.07 \\
& $p p \rightarrow h h$ & 50.96 \\
& $t \bar{t} b \bar{b}$ & 152.77 \\
\cline { 2 - 3 } & Total & 5621.45 \\
\hline
\end{tabular}

\begin{tabular}{|c|c|c|}
\hline$(\mathrm{d})$ & Process & Events \\
\hline \multirow{5}{*}{ Background } & $b \bar{b} b \bar{b}$ & 1228.85 \\
& $b \bar{b} c \bar{c}$ & 127.17 \\
& $b \bar{b} j j$ & 219.03 \\
& $t \bar{t}$ & 63.26 \\
& $p p \rightarrow h h$ & 18.44 \\
& $t \bar{t} b \bar{b}$ & 52.97 \\
\cline { 2 - 3 } & Total & 1709.72 \\
\hline
\end{tabular}

Table 9. Respective background yields for the $b \bar{b} b \bar{b}$ channel after the BDT analyses optimised for a heavy Higgs mass of $(a) 400 \mathrm{GeV},(b) 600 \mathrm{GeV},(c) 800 \mathrm{GeV}$ and $(d) 1 \mathrm{TeV}$. The tables also list the perturbative order at which the cross-sections are considered.

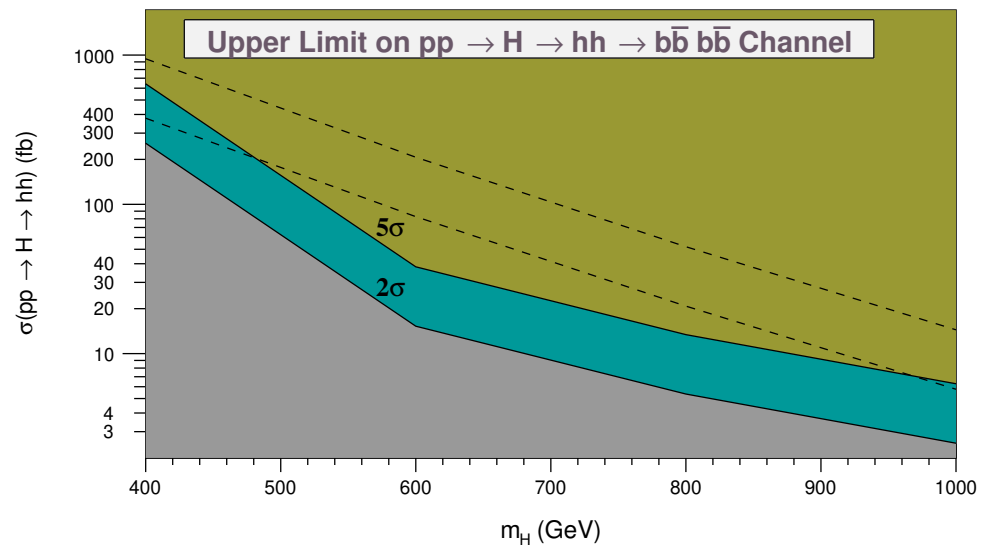

Figure 13. Upper limit on $\sigma(p p \rightarrow H \rightarrow h h)$ (fb) as a function of $m_{H}(\mathrm{GeV})$ for the $b \bar{b} b \bar{b}$ channel. The solid (dashed) lines show the $2 \sigma-5 \sigma$ band on taking $0 \%$ (5\%) systematic uncertainties.

Discussion about $\boldsymbol{m}_{\boldsymbol{H}}=\mathbf{4 0 0} \mathbf{G e V}$. The $95 \%$ and $99.7 \%$ CL upper limits on $\sigma(p p \rightarrow$ $H \rightarrow h h$ ) for the heavy Higgs with a mass around $400 \mathrm{GeV}$ is very large (256.66 fb and $641.65 \mathrm{fb}$ respectively) as compared to the other mass points, even after the BDT optimisation. The reason for this is the following. The signal efficiency for $m_{H}=400 \mathrm{GeV}$ reduces by $\sim 67 \%$ after imposing the $\Delta R_{b b}$ selection as can be seen from table 8 . Since the heavy Higgs mass $(400 \mathrm{GeV})$ is near the threshold of the non-resonant di-Higgs production, the SM-like Higgs bosons for the resonant case are produced with low $p_{T}$. This further leads to the Higgs decay products being widely separated in the $\eta-\phi$ plane and thus obviously does not satisfy our di-jet selection criteria of $\Delta R_{b b}<1.5$ within each di-jet system. With 
the sole intention of improving the sensitivity, we adopt a $\chi^{2}$ minimisation technique as described below. We define a new kinematic variable $\chi_{h h}^{2}$ for the events which do not satisfy the $\Delta R_{b b}<1.5$ selection criteria as follows

$$
\chi_{h h}^{2} \equiv \min _{\Delta R_{b b}}\left[\frac{\left(m_{\mathrm{di}-\mathrm{jet}, 1}^{2}-m_{h}^{2}\right)^{2}}{\sigma_{h 1}^{4}}+\frac{\left(m_{\mathrm{di}-\mathrm{jet}, 2}^{2}-m_{h}^{2}\right)^{2}}{\sigma_{h 2}^{4}}\right],
$$

where $m_{h}=125 \mathrm{GeV}$ and $\sigma_{h j}=0.1 \times m_{\text {di-jet }, j}$ with $j=1,2$ marks the $p_{T}$ ordering. Thus, in addition to the events satisfying $\Delta R_{b b}<1.5$, we also consider those events which contain di-jet pairs with $\Delta R$ separation between the $b$-jets to be more than 1.5. Following this, we construct the aforementioned $\chi_{h h}^{2}$ variable for each possible pair of reconstructed di-jet. The event is finally selected if the non-zero minimum value of the $\chi_{h h}^{2}$ variable is less than 50. ${ }^{4}$ Upon using this modification, the signal efficiency increases by $\sim 26 \%$ at the di-jet selection level while simultaneously increasing the dominant backgrounds like $b \bar{b} b \bar{b}$ by $\sim 5 \%$ and $t \bar{t}$ by $\sim 7 \%$. However, the limit on the upper limit of the cross-section improves to $245.75 \mathrm{fb}$ and $614.37 \mathrm{fb}$ at $95 \%$ and $99.7 \%$ CL respectively.

\subsection{The $b \bar{b} \tau \tau$ channel}

Next, we turn our attention to one of the best probes for the di-Higgs searches, viz., the $b \bar{b} \tau \tau$ channel. The intricacy and potential of this channel lies in our ability to reconstruct the $\tau$-leptons as these come with neutrinos which show up as missing transverse energy in the detector. This channel gives rise to three phenomenologically different final states, viz., $b \bar{b} \ell^{+} \ell^{-}+\mathbb{E}_{T}, b \bar{b} \ell \tau_{h}+\mathbb{E}_{T}$ and $b \bar{b} \tau_{h} \tau_{h}+\mathbb{E}_{T}$. In this work, we will only consider the last category, i.e., the one with the fully hadronic $\tau$ decays. The hadronically decayed $\tau$-leptons are termed as $\tau$-hadrons or $\tau$-jets which may either contain one (one-pronged) or three (threepronged) charged particle(s) inside the jet cone. Thus, it is essential to tag these $\tau$-jets in order to segregate them from regular QCD jets ensuing from the various backgrounds that we will discuss below. We will not discuss the fully leptonic case here as from our previous analysis [105] we know that the sensitivity is extremely low even at the HL-LHC.

We generate two different samples for the dominant $t \bar{t}$ background (figure 14), where either both the $W$-bosons decay to jets or where one decays into a lepton (viz. $e^{ \pm}, \mu^{ \pm}$or $\tau^{ \pm}$) and the other to a pair of jets. The QCD-QED background $\tau \tau b \bar{b}$ also contributes significantly. Besides, we also generate the subdominant backgrounds which include $t \bar{t} h, t \bar{t} W$, $t \bar{t} Z, b \bar{b} h, Z h$ and the non-resonant Higgs pair production i.e., $g g \rightarrow h h$. We simulate the $Z h$ background upon considering two processes where in one case the $Z$-boson decays to a pair of bottom quarks and the Higgs boson decays to a pair of $\tau$-leptons and in the other the decays are reversed. Moreover, we also generate the dominant fake background for the hadronic channel in the form of $b \bar{b} j j$ (figure 14), where the light jets can be fake $\tau$-tagged

\footnotetext{
${ }^{4}$ We checked our results upon choosing $\chi_{h h}^{2}$ both higher and lower than 50 . For higher values, the signal yield increases but the background increases at a higher rate, generating a weaker limit. On the other hand, upon lowering the $\chi_{h h}^{2}$ value below 50 , the signal yield decreases substantially. This makes the value 50 an ideal and optimal choice.
} 


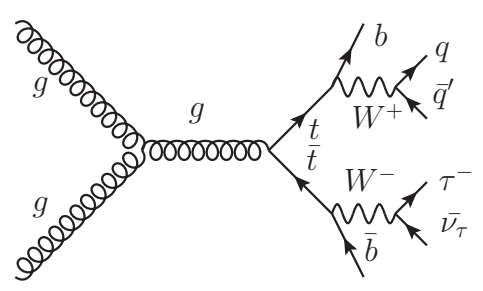

(a)

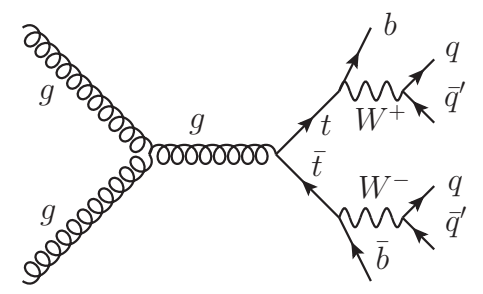

(b)

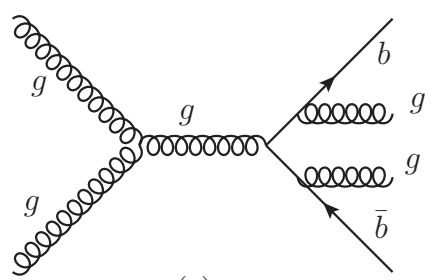

$(c)$

Figure 14. Feynman diagrams for the dominant (a) semi-leptonic $t \bar{t}$, (b) fully hadronic $t \bar{t}$ and (d) $b \bar{b} j j$ (where jet (j) can fake as $\tau$ jet) background for the $b \bar{b} \tau \tau$ channel.

jets. ${ }^{5}$ We detail the generation level cuts for these various backgrounds in appendix A. Following the generation level cuts, we further apply some basic cuts on the signal and background samples in order to ensure a common kinematic phase space. The $b / \tau$-jets and the leptons $(e, \mu)$ are required to lie within $|\eta|<2.5$ and have $p_{T, b / \tau(\ell)}>20(10) \mathrm{GeV}$. The light jets must satisfy $p_{T, j}>20 \mathrm{GeV}$ and $\left|\eta_{j}\right|<4.5$. The minimum distance in the $\eta-\phi$ plane between the $b$-jets and the leptons, and also among themselves is required to be $\Delta R>0.2$. The reconstructed invariant mass of the bottom pair and the visible $\tau$ pair must obey $m_{b b / \tau \tau}>50 \mathrm{GeV}$.

\subsubsection{The $b \bar{b} \tau_{h} \tau_{h}$ channel}

In this sub-section we briefly outline the prospects of searching for the heavy Higgs in the $b \bar{b} \tau_{h} \tau_{h}$ final state. In doing so, we select events containing exactly two $b$-tagged jets and two $\tau$-tagged jets alongside the cuts described above. Having seen the strength of the multivariate analyses for this channel, in ref. [105], rather than opting for the classical cut-based analysis, we perform a BDT analysis with the following 13 variables with the maximal discerning capability:

$$
\begin{aligned}
& p_{T, b b}, m_{b b}, p_{T, \tau_{h} \tau_{h}}, \Delta R_{\tau_{h} \tau_{h}}, \Delta \phi_{\tau_{h 1} \mathbb{E}_{T}}, \Delta \phi_{\tau_{h 2} E_{T}}, \\
& M_{T}, m_{T 2}, p_{T, \text { tot }}, m_{\text {tot }}, m_{\text {eff }}, \Delta R_{b_{1} \tau_{h 1}}, \Delta R_{b b, \tau_{h} \tau_{h}}
\end{aligned}
$$

where, $M_{T}$ is the transverse mass of the $h \rightarrow \tau \tau$ system, ${ }^{6} p_{T, \text { tot }}$ and $m_{\text {tot }}$ are respectively the transverse momenta and mass of the full visible system and $m_{\mathrm{eff}}$ is the scalar sum of

\footnotetext{
${ }^{5}$ The $\tau$-leptons decay hadronically (each with a branching fraction of $\sim 65 \%$ ) leading to jets in the final state. In our analyses, we use the $\tau$-tagging method as discussed in ref. [154].

${ }^{6}$ For this whole section, we use the conventional definition [155] of $M_{T}^{2}=\left(\sum_{i} E_{T, i}\right)^{2}-\left(\sum_{i} \vec{p}_{T, i}\right)^{2}$, where $i$ runs over the relevant objects. In section 4 , we use a modified definition of $\stackrel{i}{M_{T}}$.
} 


\begin{tabular}{|c|c|c|c|}
\hline (a) & Process & Order & Events \\
\hline \multirow{11}{*}{ Background } & $t \bar{t}$ had & NNLO [153] & 662.20 \\
\hline & $t \bar{t}$ semi-lep & NNLO [153] & 5366.58 \\
\hline & $\tau \tau b \bar{b}$ & LO & 3143.59 \\
\hline & $t \bar{t} h$ & NLO [149] & 296.20 \\
\hline & $t \bar{t} Z$ & NLO [156] & 141.56 \\
\hline & $t \bar{t} W$ & NLO [157] & 33.50 \\
\hline & $p p \rightarrow h h$ & NNLO [148] & 50.46 \\
\hline & $b \bar{b} h$ & NNLO & 2.36 \\
\hline & $Z h$ & NNLO & 132.88 \\
\hline & $b \bar{b} j j$ & LO & 9558.83 \\
\hline & \multicolumn{2}{|c|}{ Total } & 19388.16 \\
\hline
\end{tabular}

\begin{tabular}{|c|c|c|}
\hline (b) & Process & Events \\
\hline & $t \bar{t}$ had & 126.60 \\
& $t \bar{t}$ semi-lep & 884.05 \\
$\tau \tau b \bar{b}$ & 633.83 \\
& $t \bar{t} h$ & 90.79 \\
& $t \bar{t} Z$ & 57.06 \\
Background & $t \bar{t} W$ & 3.28 \\
& $p p \rightarrow h h$ & 29.85 \\
& $b \bar{b} h$ & 0.36 \\
& $Z h$ & 60.94 \\
& $b \bar{b} j j$ & 3303.97 \\
\hline & Total & 5190.73 \\
\hline
\end{tabular}

\begin{tabular}{|c|c|c|}
\hline$(\mathrm{c})$ & Process & Events \\
\hline \multirow{5}{*}{ Background } & $t \bar{t}$ had & 97.38 \\
& $t \bar{t}$ semi-lep & 498.06 \\
& $\tau \tau b \bar{b}$ & 379.98 \\
& $t \bar{t} h$ & 60.52 \\
& $t \bar{t} Z$ & 57.06 \\
& $t \bar{t} W$ & 6.57 \\
& $p p \rightarrow h h$ & 12.87 \\
& $b \bar{b} h$ & 0.29 \\
& $Z h$ & 24.50 \\
& $b \bar{b} j j$ & 1639.38 \\
\cline { 2 - 3 } & Total & 2776.61 \\
\hline
\end{tabular}

\begin{tabular}{|c|c|c|}
\hline (d) & Process & Events \\
\hline & $t \bar{t}$ had & 48.69 \\
& $t \bar{t}$ semi-lep & 460.70 \\
& $\tau \tau b \bar{b}$ & 319.31 \\
& $t \bar{t} h$ & 70.61 \\
& $t \bar{t} Z$ & 48.29 \\
Background & $t \bar{t} W$ & 11.82 \\
& $p p \rightarrow h h$ & 9.92 \\
& $b \bar{b} h$ & 0.24 \\
& $Z h h$ & 19.34 \\
& $b \bar{b} j j$ & 2068.14 \\
\cline { 2 - 3 } & Total & 3057.06 \\
\hline
\end{tabular}

Table 10. Background yields after the BDT analysis for heavy Higgs mass of (a) $400 \mathrm{GeV}$, (b) $600 \mathrm{GeV},(c) 800 \mathrm{GeV}$ and $(d) 1000 \mathrm{GeV}$ for the $b \bar{b} \tau_{h} \tau_{h}$ channel.

the transverse mass of all the visible products plus $\mathbb{E}_{T}$. The rest of the variables have usual definitions. The top five variables are shown in figure 15. As can be seen, the $m_{T 2}$ variable is particularly useful for heavier Higgs masses as it can be used to completely eradicate the $t \bar{t}$ background. We train the signal and background samples and they are optimised for each benchmark signal point. We list the background events after optimising the BDT and imposing the cut for four values of $m_{H}$, in table 10. Finally, we show the upper limit on the heavy Higgs production cross-section (assuming $\operatorname{BR}(H \rightarrow h h)=100 \%$ ) in figure 16. The 95\% CL upper limit on the cross-section between $m_{H}=600 \mathrm{GeV}$ and $1 \mathrm{TeV}$ varies between $67.34 \mathrm{fb}$ and $39.56 \mathrm{fb}$. With $5 \%$ systematic uncertainty, the limits become $251.76 \mathrm{fb}$ and $116.30 \mathrm{fb}$ respectively.

\subsection{The $b \bar{b} W W^{*}$ channel}

In this section, we consider the situation where a heavy scalar decays to a pair of SM-like Higgs bosons with one of them decaying to a pair of $b$-quarks and the other to $W W^{*}$, leading to three possible final states depending on the decays of the $W$-bosons. We perform our analyses for the fully leptonic (leptons at this stage include $e, \mu, \tau$ ) and the semi-leptonic 

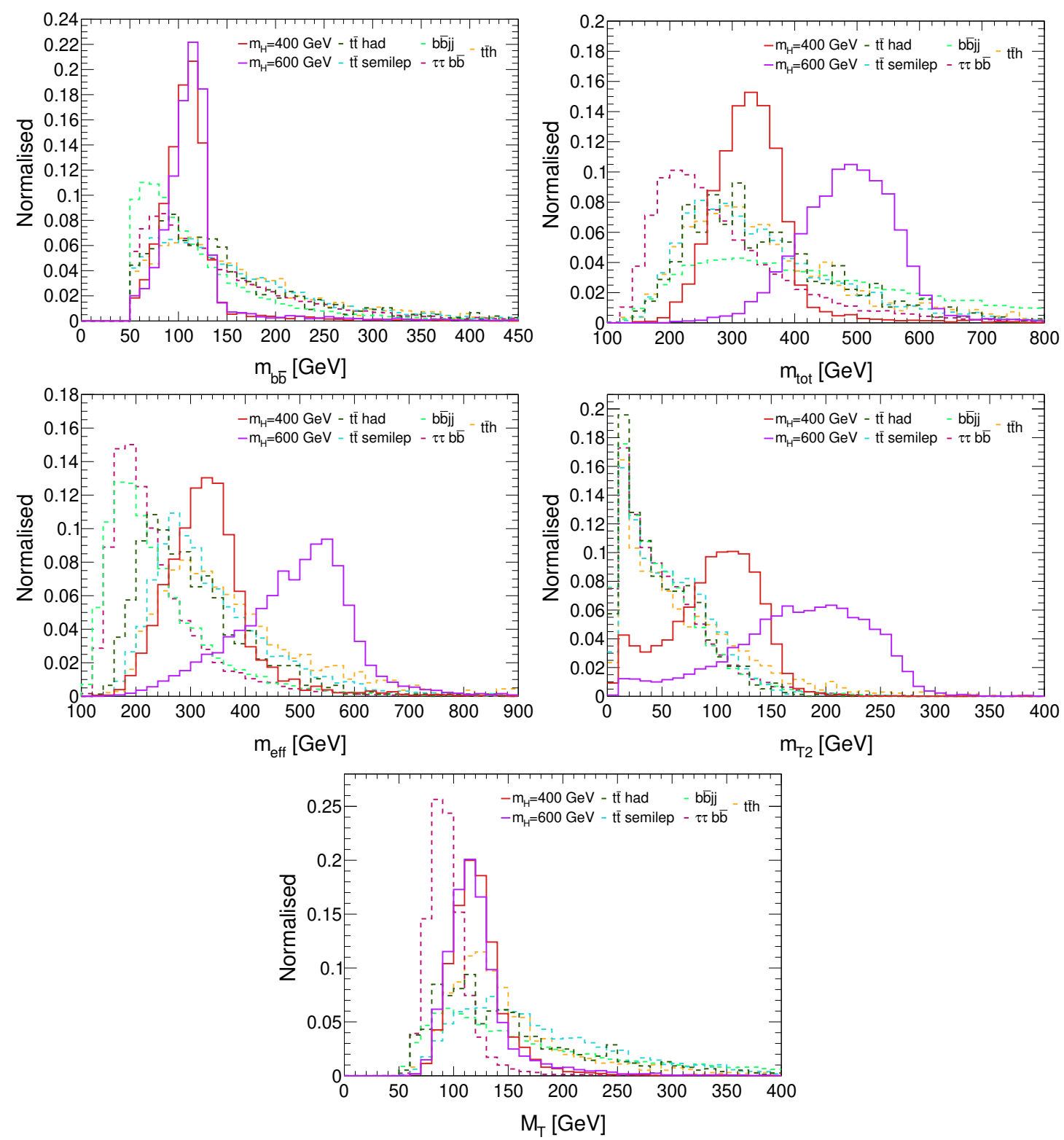

Figure 15. The $m_{b b}, m_{\mathrm{tot}}, m_{\mathrm{eff}}, m_{T 2}$ and $M_{T}$ distributions for heavy Higgs masses of $m_{H}=400$ and $600 \mathrm{GeV}$ with dominant backgrounds. Here the heavy Higgs boson is searched for in the $b \bar{b} \tau_{h} \tau_{h}$ final state. The distributions are shown after imposing the basic trigger cuts.

channels. We avoid studying the fully hadronic mode as the signal will be overwhelmed by the huge QCD background.

The dominant contribution to the background (figure 17) for both the channels mentioned above comes from top pair production because of its large production cross-section. We generate this background where either or both the $W$-bosons decay leptonically. The fully hadronic $t \bar{t}$ mode is not considered as a potential background as the fake rate for $j \rightarrow \ell$ is negligible for all practical purposes. The fully leptonic $t \bar{t}$ background contributes to the fully leptonic channel final state whereas for the semi-leptonic scenario, the con- 


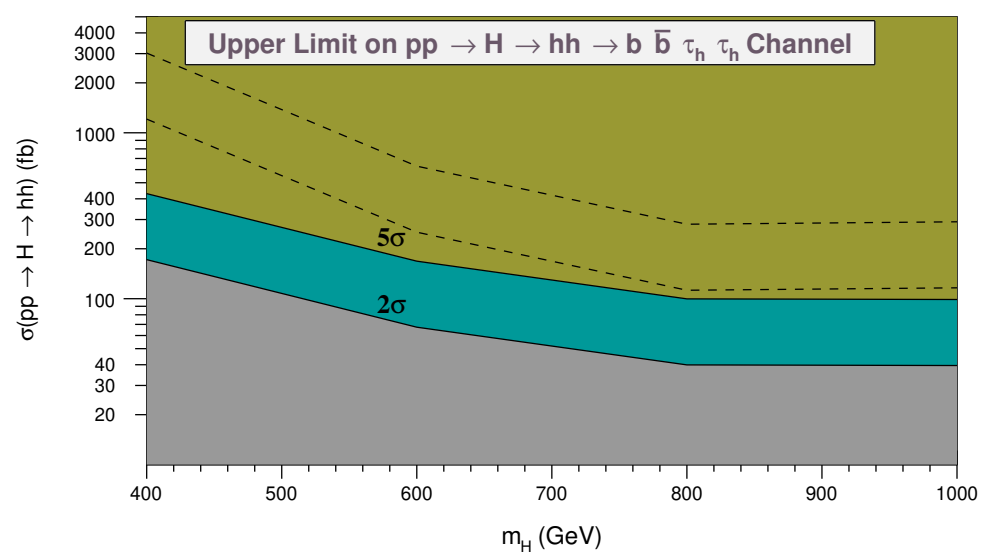

Figure 16. Upper limit on $\sigma(p p \rightarrow H \rightarrow h h)(\mathrm{fb})$ as a function of $m_{H}(\mathrm{GeV})$ for the $b \bar{b} \tau_{h} \tau_{h}$ channel. The solid (dashed) lines show the $2 \sigma-5 \sigma$ band on taking $0 \%(5 \%)$ systematic uncertainties.

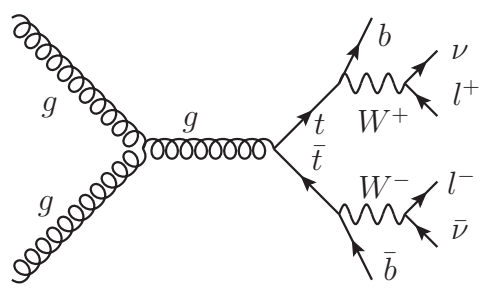

$(a)$

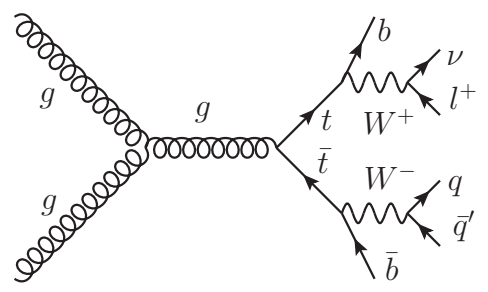

(b)

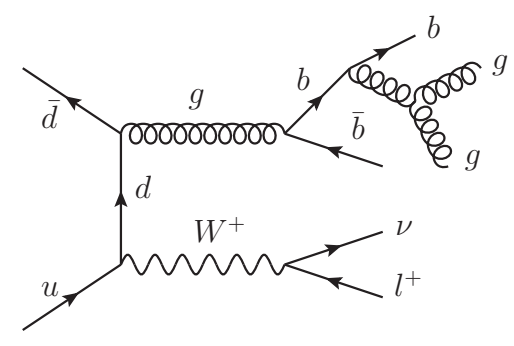

(c)

Figure 17. Feynman diagrams for (a) leptonic $t \bar{t}$, (b) semi-leptonic $t \bar{t}$ and (c) $W b \bar{b}+$ jets background for the $b \bar{b} W W^{*}$ channel. Here, $l$ refers to $e, \mu$ and $\tau$ lepton.

tribution comes from both the fully-leptonic as well as the semi-leptonic $t \bar{t}$. The second most dominant background for the semi-leptonic channel is $W b \bar{b}+$ jets, where the $W$ boson decays leptonically $(e, \mu, \tau)$. We generate this background upon merging with two additional jets by exploiting the MLM merging scheme [147]. While generating the $W b \bar{b}+$ jets background we ensure that there is no double counting ensuing from the semi-leptonic $t \bar{t}$ background. Besides the aforementioned backgrounds, a significant contribution also comes from the $\ell^{+} \ell^{-} b \bar{b}$ production where $\ell$ refers to $e, \mu$ and $\tau$. Finally, we also consider the subdominant backgrounds viz., $t \bar{t} h, t \bar{t} Z, t \bar{t} W$ and the non-resonant $g g \rightarrow h h$.

In this subsection and the following section (section 3), the top-pair production is the dominant background. Thus, the reconstruction of the top quarks is a very powerful tool 

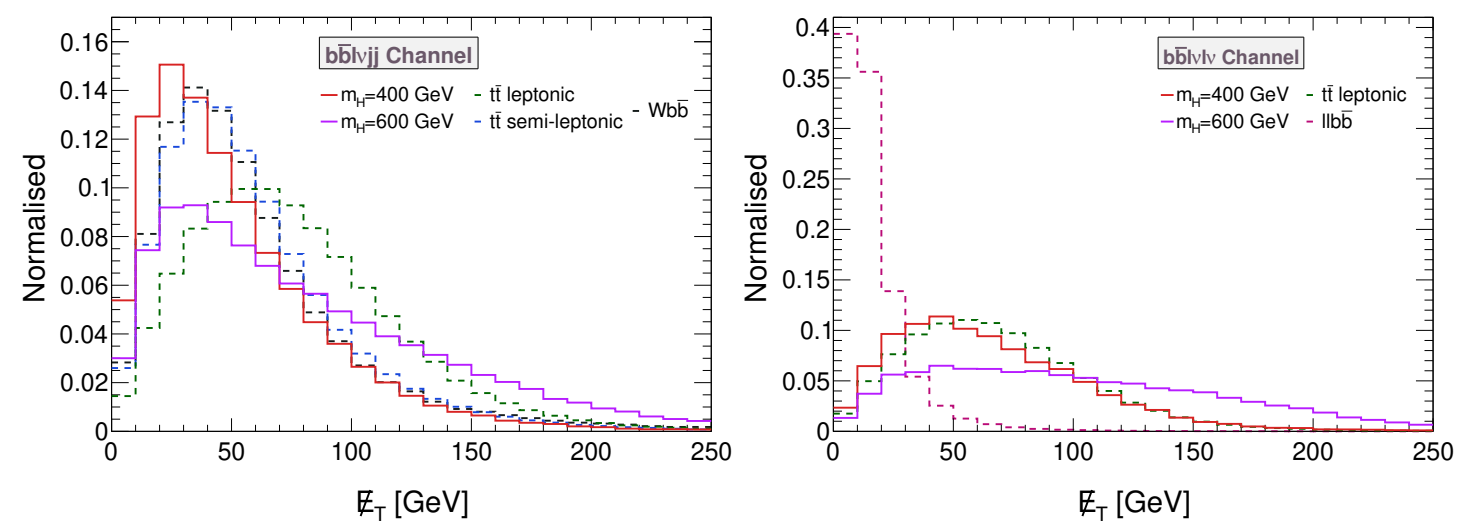

Figure 18. The $\mathbb{E}_{T}$ distributions for the $1 \ell$ and $2 \ell$ categories for $m_{H}=400$ and $600 \mathrm{GeV}$ with dominant backgrounds. Here the heavy Higgs boson is searched for in the $b b W W^{*}$ channel. The distributions are shown after imposing the basic trigger cuts.

in order to reduce the contribution from this background. For the semi-leptonic case, the only source of missing transverse energy, $\mathbb{E}_{T},{ }^{7}$ arises from the neutrino of the leptonically decaying $W$-boson from the top decay. We reconstruct the top from its decay products. ${ }^{8}$ The quadratic equation gives two possible solutions for the neutrino $p_{z}$. Besides, because there are two $b$-jets in the final state, we get four possible choices for the mass of the leptonically decaying top. We use these variables during our analysis. After reconstructing both the tops, we reconstruct the total system from all the final state particles. We also use this later in section 3 which exhibits the same final state. These variables help us greatly in reducing the semi-leptonic $t \bar{t}$ background for high values of $m_{H}$.

Before embarking on the final analysis, we impose a common set of trigger cuts for both the leptonic and the semi-leptonic channels. The $p_{T},|\eta|$ and $\Delta R$ cuts for the various objects are discussed in subsection 2.3 and also in appendix A. Furthermore, we require generation-level cuts on the invariant mass of b-jets, viz., $m_{b b}>50 \mathrm{GeV}$. The selected events are also require to have $\mathbb{E}_{T}>40 \mathrm{GeV}$ upon scrutinising the distribution. The $\mathbb{E}_{T}$ distribution for the $1 \ell$ and $2 \ell$ cases are shown in figure 18 . Finally, we perform separate multivariate analyses for the two final states upon using the BDTD algorithm. While training samples for both the leptonic and semi-leptonic analyses, we only consider the $t \bar{t}$ background since it constitutes the bulk of the total background. This training is used for testing all other backgrounds which are subdominant in front of $t \bar{t}$.

\subsubsection{The $2 \ell 2 b+\mathbb{E}_{T}$ channel}

For the fully leptonic final state, we select events with exactly two $b$-tagged jets, and two isolated leptons having opposite charge meeting the trigger criteria as mentioned above. We choose the following set of kinematic variables in order to perform the multivariate

\footnotetext{
${ }^{7}$ To incorporate the $\mathbb{E}_{T}$ smearing, we use the standard module of the Delphes ATLAS card.

${ }^{8}$ First the $W$-boson mass is reconstructed in order to attain the $p_{Z}$ component of the neutrino.
} 

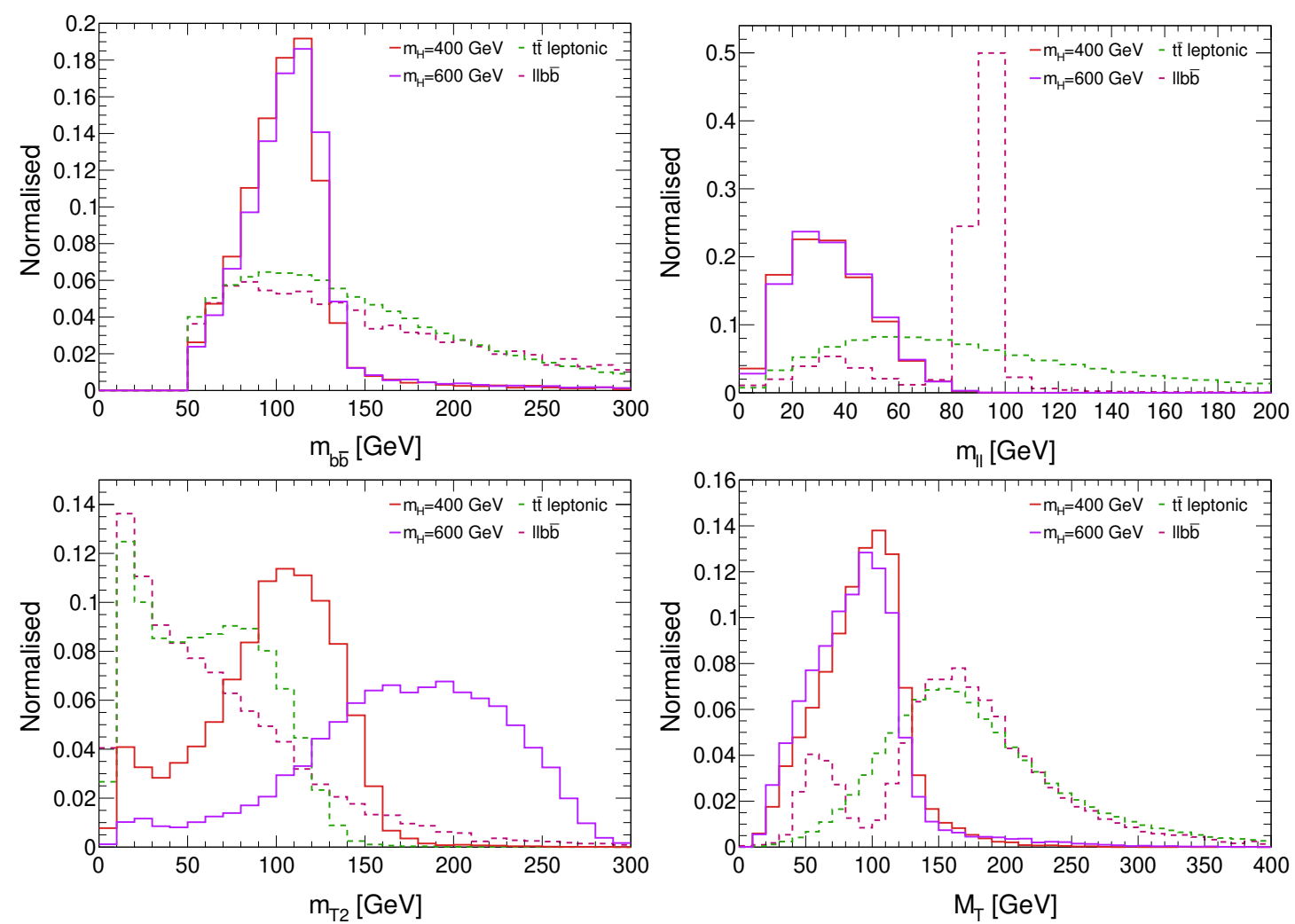

Figure 19. The $m_{b b}, m_{\ell \ell}, m_{T 2}$ and $M_{T}$ distributions for the $2 \ell$ category for $m_{H}=400$ and $600 \mathrm{GeV}$ with dominant backgrounds. Here the heavy Higgs boson is searched for in the $b \bar{b} W W^{*}$ channel. The distributions are shown before the multivariate analysis.

analysis:

$p_{T, b b}, \eta_{b b}, \phi_{b b}, m_{b b}, \Delta R_{b b}, \Delta \phi_{b b}, p_{T, \ell \ell}, \eta_{\ell \ell}, \phi_{\ell \ell}, m_{\ell \ell}, \Delta R_{\ell \ell}$,

$M_{T}, m_{T 2}, m_{\mathrm{tot}}, p_{T, \mathrm{tot}}, \phi_{\mathrm{tot}}, m_{\mathrm{eff}}, \Delta R_{b_{1} \ell_{2}}, \Delta R_{b b, \ell \ell}, p_{T, \ell_{2}}$,

where, $M_{T}$ is the transverse mass of the SM-like Higgs decaying to $W$-bosons. The rest of the variables have either been defined before or have usual meaning. The top four variables are shown in figure 19. The signal distributions are significantly different from the various backgrounds.

Finally, in table 11, we summarise the number of background events after imposing the optimised cut on the BDT variable. Like in the other channels, we impose an upper limit on $\sigma(p p \rightarrow H \rightarrow h h)$ as a function of the heavy Higgs mass. This is shown in figure 20 . The 95\% CL upper limit varies between $67.41 \mathrm{fb}$ and $26.18 \mathrm{fb}(357.51 \mathrm{fb}$ and $82.21 \mathrm{fb}$ with $5 \%$ systematic uncertainty) within $600 \mathrm{GeV}<m_{H}<1 \mathrm{TeV}$ and is somewhat weaker than the channels discussed earlier owing to smaller $S / B$.

\subsubsection{The $1 \ell 2 b 2 j+\mathbb{E}_{T}$ channel}

Finally, we discuss the potential of the semi-leptonic final state as well. We require events with exactly two $b$-tagged jets, one isolated lepton and at least two light jets satisfying 


\begin{tabular}{|c|c|c|c|}
\hline (a) & Process & Order & Events \\
\hline & $t \bar{t}$ lep & NNLO [153] & 356309.30 \\
& $t \bar{t} h$ & NLO [149] & 1310.44 \\
& $t \bar{t} Z$ & NLO [156] & 1264.20 \\
& $t \bar{t} W$ & NLO [157] & 627.97 \\
Background & $p p \rightarrow h h$ & NNLO [148] & 90.72 \\
& $\ell \ell b \bar{b}$ & LO & 5013.31 \\
\cline { 2 - 4 } & \multicolumn{2}{|c|}{ Total } & 364615.94 \\
\hline
\end{tabular}

\begin{tabular}{|c|c|c|}
\hline$(\mathrm{b})$ & Process & Events \\
\hline \multirow{5}{*}{ Background } & $t \bar{t}$ lep & 7056.45 \\
& $t \bar{t} h$ & 322.80 \\
& $t \bar{t} Z$ & 640.88 \\
& $t \bar{t} W$ & 114.29 \\
& $p p \rightarrow h h$ & 37.75 \\
& $\ell \ell b \bar{b}$ & 2678.57 \\
\cline { 2 - 3 } & Total & 10850.74 \\
\hline
\end{tabular}

\begin{tabular}{|c|c|c|}
\hline$(\mathrm{c})$ & Process & Events \\
\hline & $t \bar{t}$ lep & 11954.46 \\
& $t \bar{t} h$ & 328.30 \\
& $t \bar{t} Z$ & 812.07 \\
& $t \bar{t} W$ & 185.24 \\
Background & $p p \rightarrow h h$ & 20.38 \\
& $\ell \ell b \bar{b}$ & 3233.14 \\
\cline { 2 - 3 } & Total & 16533.59 \\
\hline
\end{tabular}

\begin{tabular}{|c|c|c|}
\hline$(\mathrm{d})$ & Process & Events \\
\hline \multirow{5}{*}{ Background } & $t \bar{t}$ lep & 1286.76 \\
& $t \bar{t} h$ & 135.72 \\
& $t \bar{t} Z$ & 386.28 \\
& $t \bar{t} W$ & 49.27 \\
& $p p \rightarrow h h$ & 10.68 \\
& $\ell \ell b \bar{b}$ & 1674.80 \\
\cline { 2 - 3 } & Total & 3543.51 \\
\hline
\end{tabular}

Table 11. Respective background yields for the $2 \ell+2 b+\mathbb{E}_{T}$ channel after the BDT analyses optimised for $m_{H}=(a) 400 \mathrm{GeV},(b) 600 \mathrm{GeV},(c) 800 \mathrm{GeV}$ and $(d) 1 \mathrm{TeV}$. The tables also list the perturbative order at which the cross-sections are considered.

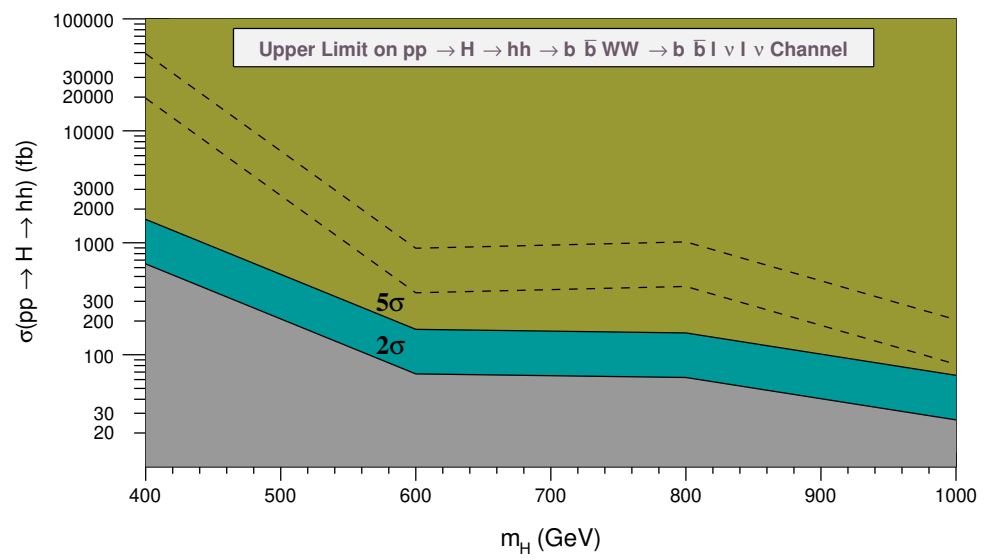

Figure 20. Upper limit on $\sigma(p p \rightarrow H \rightarrow h h)(\mathrm{fb})$ as a function of $m_{H}(\mathrm{GeV})$ for the $2 \ell+2 b+\mathbb{E}_{T}$ channel. The solid (dashed) lines show the $2 \sigma-5 \sigma$ band on taking $0 \%$ (5\%) systematic uncertainties.

the trigger criteria discussed earlier. Besides, we consider the same set of cuts as for the dileptonic channel before performing the multivariate analysis. We find the following kinematic variables to have the best discriminatory power and use them for our multivariate analysis:

$$
\begin{aligned}
& \eta_{b b}, m_{b b}, m_{t}, m_{j j}, \Delta R_{j j}, \Delta R_{\ell, j j}, M_{T}, m_{T 2}, \\
& m_{b b j_{1}}, m_{t 11}, m_{t 12}, p_{T, \ell \nu}, p_{T, b_{1}}, p_{T, \ell_{1}}, p_{T, j_{1}}
\end{aligned}
$$

where, $m_{t}$ is the transverse mass of the leptonically decaying $W$-boson. $\Delta R_{\ell, j j}$ is the distance in the $\eta-\phi$ plane between the system comprising of the two hardest jets and the 

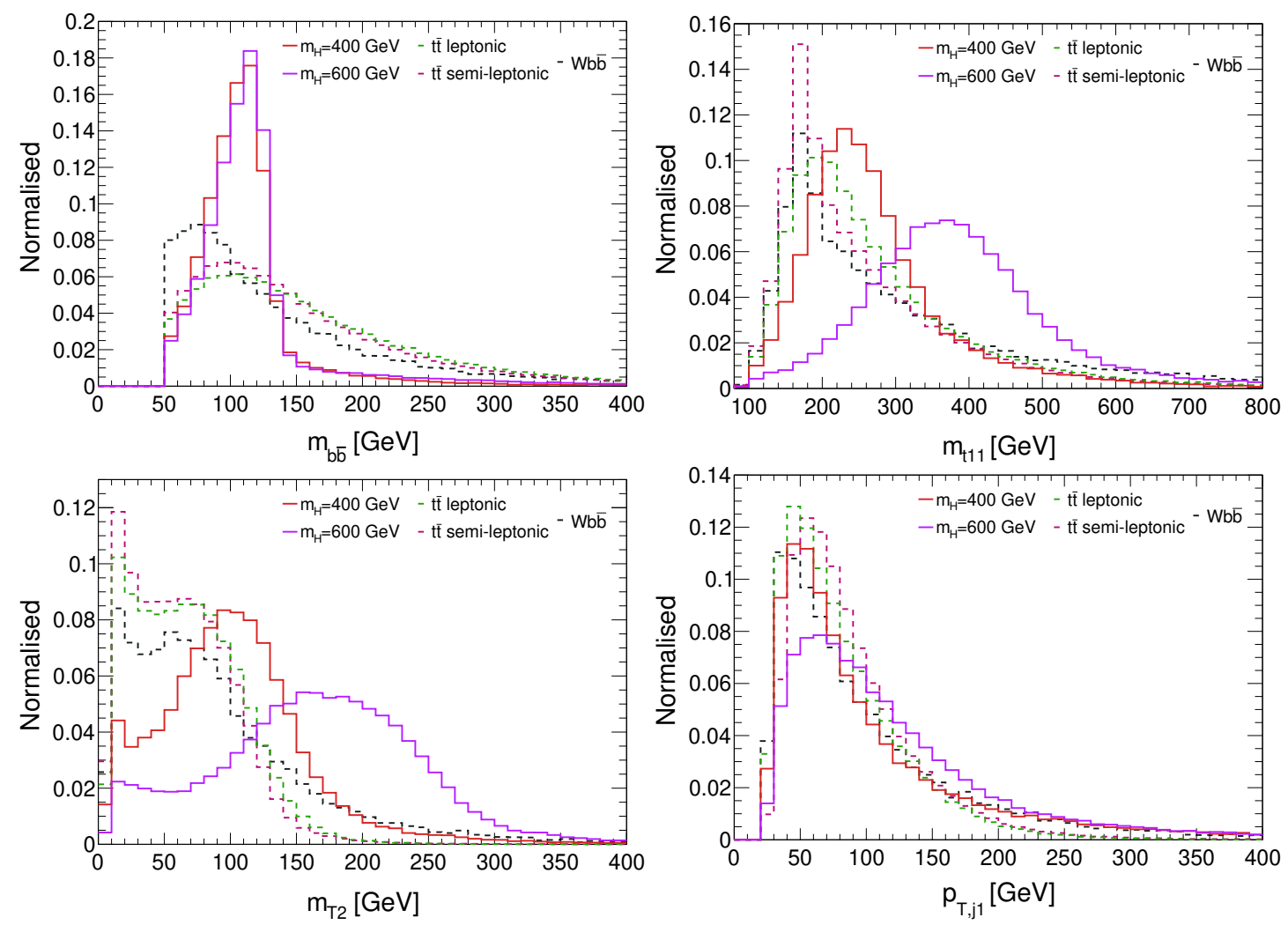

Figure 21. The $m_{b b}, m_{t 11}, m_{T 2}$ and $p_{T, j_{1}}$ distributions for the $1 \ell$ category for $m_{H}=400$ and $600 \mathrm{GeV}$ with dominant backgrounds. Here the heavy Higgs boson is searched for in the $b \bar{b} W W^{*}$ channel. The distributions are shown before the multivariate analysis.

lepton. $m_{b b j_{1}}$ refers to the invariant mass of the two $b$-tagged jets and the hardest $p_{T}$ jet. The reconstructed transverse momentum of the leptonically decaying $W$-boson is denoted as $p_{T, \ell \nu} \cdot m_{t i j}$ is the mass of the leptonically decaying top quark with the reconstruction procedure outlined before. The first index $i=1,2$ indicates the $p_{T}$ ordering of the $b$ jet. The second index $j=1,2$ refers to the choice of the $z$-component of the neutrino momentum. The other variables have usual definitions. The best discriminatory variables are listed in figure 21. However, we can see that the separation power for the $1 \ell$ category is significantly less compared to its $2 \ell$ counterpart.

Coming to the results, table 12 summarises the background yields after the BDT cut. The upper limit on $\sigma(p p \rightarrow H \rightarrow h h)$ as a function of $m_{H}$ are shown in figure 22. The limits are considerably weak in this channel.

\subsection{The $\gamma \gamma W W^{*}$ channel}

After the $b \bar{b} \gamma \gamma$ channel this is the second most cleanest channel in terms of the final state particles but with the pitfall of having very low event rate. In this channel, one of the SMlike Higgs decays to a pair of photons and the other to lepton(s) through $h \rightarrow W W^{*}$. Similar to the $b \bar{b} W W^{*}$ analysis in subsection 2.4, here also we divide the channel into the leptonic and semi-leptonic category. Because of the relatively clean final states, these channels have 


\begin{tabular}{|c|c|c|}
\hline (a) & Process & Events \\
\hline \multirow{7}{*}{ Background } & $t \bar{t}$ semi-lep & 9740640.28 \\
& $t \bar{t}$ lep & 1614225.57 \\
& $W b \bar{b}+$ jets [LO] & 569181.53 \\
& $t \bar{t} h$ & 28364.75 \\
& $t \bar{t} Z$ & 24846.06 \\
& $t \bar{t} W$ & 16935.36 \\
& $p p \rightarrow h h$ & 318.61 \\
& $\ell \ell b \bar{b}$ & 20252.87 \\
\cline { 2 - 3 } & Total & 12014765.03 \\
\hline
\end{tabular}

\begin{tabular}{|c|c|c|}
\hline (b) & Process & Events \\
\hline & $t \bar{t}$ semi-lep & 280140.51 \\
& $t \bar{t}$ lep & 40221.77 \\
& $W b \bar{b}+$ jets & 106228.20 \\
& $t \bar{t} h$ & 3804.77 \\
Background & $t \bar{t} Z$ & 2952.00 \\
& $t \bar{t} W$ & 1958.78 \\
& $p p \rightarrow h h$ & 87.93 \\
& $\ell \ell b \bar{b}$ & 1985.36 \\
\cline { 2 - 3 } & Total & 437379.32 \\
\hline
\end{tabular}

\begin{tabular}{|c|c|c|}
\hline$(\mathrm{c})$ & Process & Events \\
\hline \multirow{7}{*}{ Background } & $t \bar{t}$ semi-lep & 169281.37 \\
& $t \bar{t}$ lep & 19965.61 \\
& $W b \bar{b}+$ jets & 91249.74 \\
& $t \bar{t} h$ & 2519.09 \\
& $t \bar{t} Z$ & 2261.73 \\
& $t \bar{t} W$ & 1630.34 \\
& $p p \rightarrow h h$ & 42.35 \\
& $\ell \ell b \bar{b}$ & 848.49 \\
\cline { 2 - 3 } & Total & 287798.72 \\
\hline
\end{tabular}

\begin{tabular}{|c|c|c|}
\hline$(\mathrm{d})$ & Process & Events \\
\hline \multirow{7}{*}{ Background } & $t \bar{t}$ semi-lep & 138443.80 \\
& $t \bar{t}$ lep & 19342.98 \\
& $W b \bar{b}+$ jets & 77935.55 \\
& $t \bar{t} h$ & 1842.32 \\
& $t \bar{t} Z$ & 1919.34 \\
& $t \bar{t} W$ & 1399.78 \\
& $p p \rightarrow h h$ & 21.38 \\
& $\ell \ell b \bar{b}$ & 571.21 \\
\cline { 2 - 3 } & Total & 241476.36 \\
\hline
\end{tabular}

Table 12. Respective background yields for the $1 \ell+2 j+2 b+\mathbb{E}_{T}$ channel after the BDT analyses optimised for $m_{H}=(a) 400 \mathrm{GeV}$, (b) $600 \mathrm{GeV},(c) 800 \mathrm{GeV}$ and $(d) 1 \mathrm{TeV}$. The various orders of the signal and backgrounds are same as in table 11.

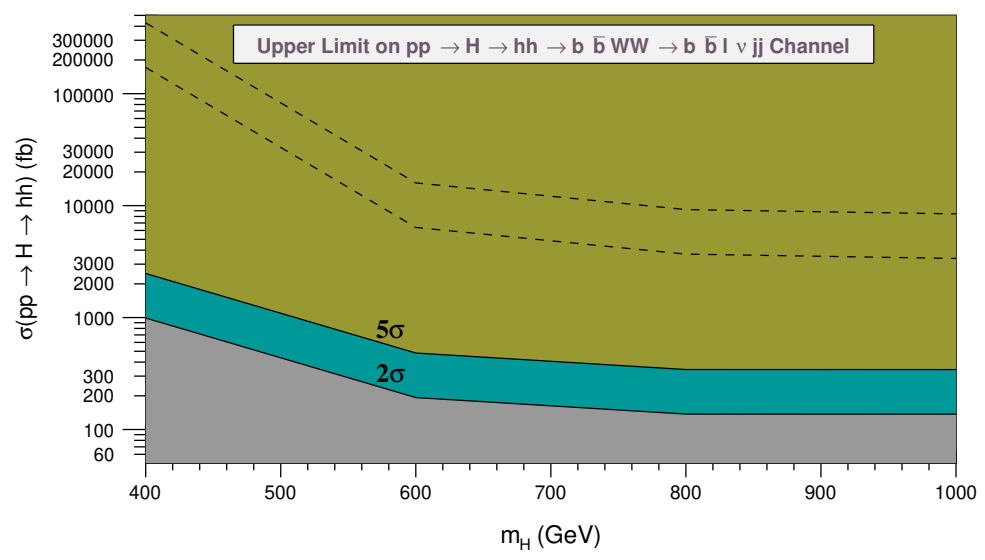

Figure 22. Upper limit on $\sigma(p p \rightarrow H \rightarrow h h)(\mathrm{fb})$ as a function of $m_{H}(\mathrm{GeV})$ for the $1 \ell+2 j+2 b+\mathbb{E}_{T}$ channel. The solid (dashed) lines show the $2 \sigma-5 \sigma$ band on taking $0 \%$ (5\%) systematic uncertainties. 


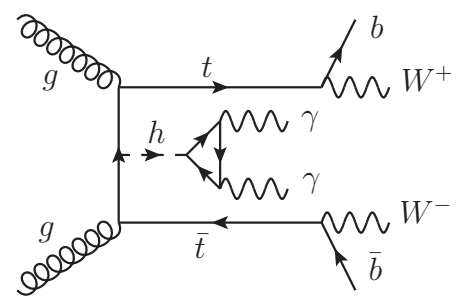

(a)

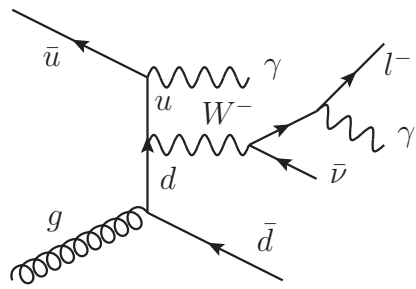

(c)

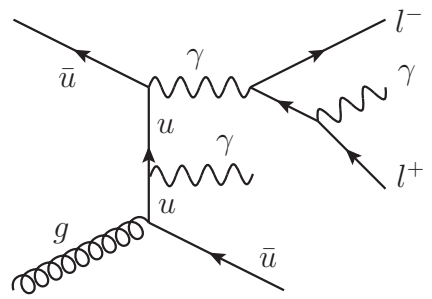

$(b)$

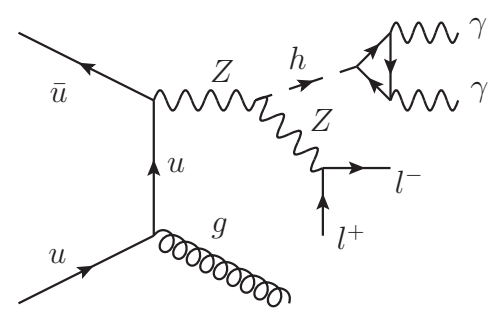

(d)

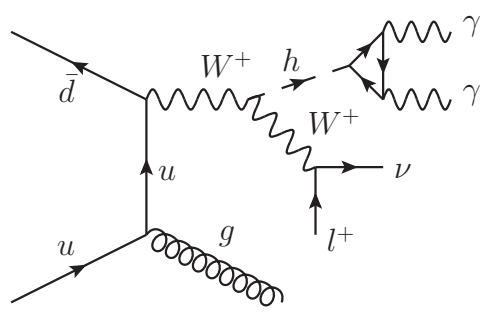

(e)

Figure 23. Feynman diagrams for (a) $t \bar{t} h$, (b) $\ell \ell \gamma \gamma+$ jets, (c) $\ell \nu \gamma \gamma+$ jets, (d) $Z h+$ jets and (e) $W h+$ jets background for the $\gamma \gamma W W^{*}$ channel.

low contaminations due to backgrounds. We simulate the $Z h$ and $W h$ backgrounds upon merging with two additional jets (the definition of jet is given in subsection 2.1). Here we decay the $Z$ - and the $W$-bosons leptonically $(e, \mu, \tau)$. The $W h$ channel contributes only to the semi-leptonic category. Besides, there are $\ell \nu \gamma \gamma$ and $\ell \ell \gamma \gamma$ productions coming from $\gamma^{*}$, diagrams containing triple and quartic gauge boson interactions and various other diagrams not involving the Higgs. We generate these two backgrounds upon merging with an additional jet and using the same scheme as before. Next, we also consider the $t \bar{t} h$ background with Higgs-boson decayed to a pair of photons. Finally, we also consider the SM Higgs pair production which is subdominant. We show the Feynman diagram of the dominant backgrounds in figure 23.

Before performing the multivariate analyses, we impose the generic trigger cuts. The $p_{T},|\eta|$ and $\Delta R,{ }^{9}$ cuts are the same as has been defined in subsection 2.3. The above cuts for the photons are the same as those on the leptons. Owing to an excellent resolution for the diphoton invariant mass, we require $122 \mathrm{GeV}<m_{\gamma \gamma}<128 \mathrm{GeV}$. Finally, we also require $m_{\ell \ell}>20 \mathrm{GeV}$ because we generate the $\ell \ell \gamma \gamma$ background with this invariant mass cut at the generation level (the details of these cuts are mentioned in appendix A). We now

\footnotetext{
${ }^{9} \Delta R_{\gamma \gamma / \gamma \ell}>0.4$ and $\Delta R_{\ell \ell>0.2}$.
} 


\begin{tabular}{|c|c|c|c|}
\hline (a) & Process & Order & Events \\
\hline \multirow{5}{*}{ Background } & $t \bar{t} h$ & NLO [149] & 6.16 \\
& $Z h+$ jets & NNLO (QCD) + NLO (EW) $[149]$ & 1.28 \\
& $W h+$ jets & NNLO (QCD) + NLO (EW) $[149]$ & 11.27 \\
& $p p \rightarrow h h$ & NNLO [148] & 1.35 \\
& $\ell \nu \gamma \gamma+$ jets & LO & 3.33 \\
& $\ell \ell \gamma \gamma+$ jets & LO & $\sim 1.00$ \\
\cline { 2 - 4 } & \multicolumn{2}{|c|}{ Total } \\
\hline
\end{tabular}

\begin{tabular}{|c|c|c|}
\hline (b) & Process & Events \\
\hline & $t \bar{t} h$ & 6.94 \\
& $Z h+$ jets & 1.21 \\
& $W h+$ jets & 9.67 \\
Background & $p p \rightarrow h h$ & 1.10 \\
& $\ell \nu \gamma \gamma+$ jets & 2.25 \\
& $\ell \ell \gamma \gamma+$ jets & 0.42 \\
\hline & Total & 21.59 \\
\hline
\end{tabular}

\begin{tabular}{|c|c|c|}
\hline$(\mathrm{c})$ & Process & Events \\
\hline \multirow{5}{*}{ Background } & $t \bar{t} h$ & 3.43 \\
& $Z h+$ jets & 0.37 \\
& $W h+$ jets & 4.50 \\
& $p p \rightarrow h h$ & 0.25 \\
& $\ell \nu \gamma \gamma+$ jets & 1.10 \\
& $\ell \ell \gamma \gamma+$ jets & 0.13 \\
\cline { 2 - 3 } & Total & 9.78 \\
\hline
\end{tabular}

\begin{tabular}{|c|c|c|}
\hline$(\mathrm{d})$ & Process & Events \\
\hline \multirow{5}{*}{ Background } & $t \bar{t} h$ & 2.68 \\
& $Z h+$ jets & 0.20 \\
& $W h+$ jets & 3.38 \\
& $p p \rightarrow h h$ & 0.13 \\
& $\ell \nu \gamma \gamma+$ jets & 1.09 \\
& $\ell \ell \gamma \gamma+$ jets & 0.08 \\
\cline { 2 - 3 } & Total & 7.56 \\
\hline
\end{tabular}

Table 13. Respective background yields for the $\gamma \gamma 1 \ell 2 j+\notin_{T}$ channel after the BDT analyses optimised for $m_{H}=(a) 400 \mathrm{GeV}$, (b) $600 \mathrm{GeV},(c) 800 \mathrm{GeV}$ and $(d) 1 \mathrm{TeV}$. The tables also list the perturbative order at which the cross-sections are considered.

describe the results of the multivariate analyses for these two channels in the following two subsections.

\subsubsection{The $\gamma \gamma 1 \ell 2 j+\mathbb{E}_{T}$ channel}

Before performing the BDT analysis, we select events with exactly two isolated photons, one isolated lepton and at least two jets in the final state, which fulfils all the aforementioned trigger requirements. Like all the other channels, we consider the following variables to train our signal and background samples for the multivariate analysis:

$$
p_{T, \gamma \gamma}, \Delta R_{\gamma \gamma}, \Delta \phi_{\ell E_{T}}, m t, \Delta R_{\ell j j}, m_{\mathrm{tot}}, m_{\mathrm{eff}}, M_{T}, \Delta R_{\gamma_{1} \ell}, \Delta R_{\gamma_{2} \ell}, p_{T, \gamma_{2}}, p_{T, \ell}, \mathbb{E}_{T},
$$

where the variables carry their usual meaning. The five best variables are shown in figure 24 . The background yields after the BDT optimisation are shown in table 13. In figure 25, we show the upper limit on $\sigma(p p \rightarrow H \rightarrow h h)$ as a function of $m_{H}$. The 95\% CL upper limit changes from $220.11 \mathrm{fb}$ ( $226.74 \mathrm{fb}$ with $5 \%$ systematic) for $m_{H}=400 \mathrm{GeV}$ to $112.20 \mathrm{fb}$ (113.24fb with $5 \%$ systematic) for $m_{H}=1 \mathrm{TeV}$.

\subsubsection{The $\gamma \gamma 2 \ell+\mathbb{E}_{T}$ channel}

This is the final channel that we study for the $p p \rightarrow H \rightarrow h h$ case. We choose events with exactly two isolated photons, and two isolated leptons with opposite charge, following the trigger cuts mentioned above. Finally, we choose the following kinematic variables for the multivariate analysis:

$$
p_{T, \gamma \gamma}, \Delta R_{\gamma \gamma}, \Delta \phi_{\gamma \gamma}, m_{\ell \ell}, \Delta R_{\ell \ell}, M_{T}, m_{\mathrm{tot}}, m_{\mathrm{eff}}, \Delta R_{\gamma \gamma, \ell \ell}, p_{T, \ell 1},
$$



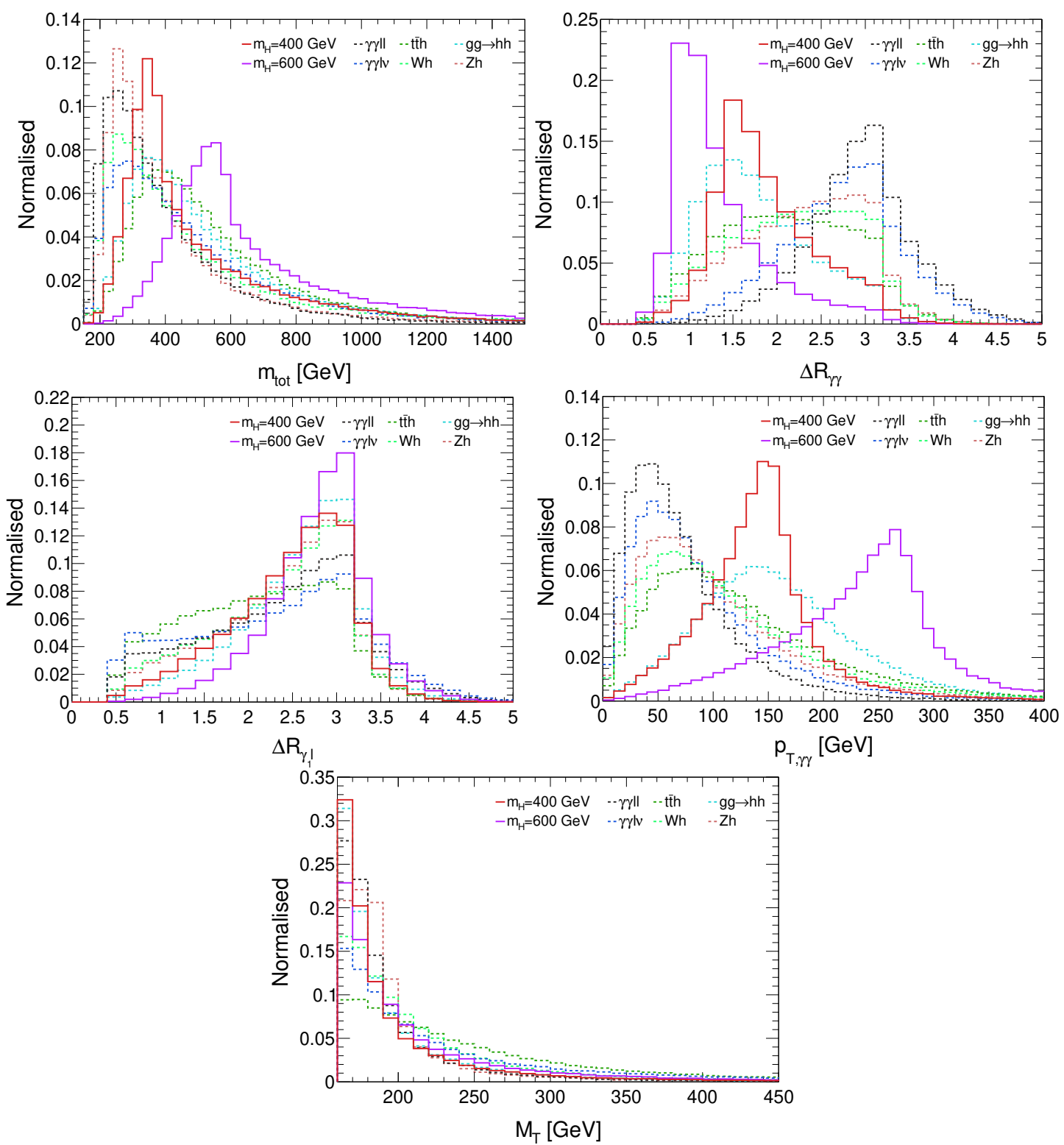

Figure 24. The $m_{\text {tot }}, \Delta R_{\gamma \gamma}, \Delta R_{\gamma_{1} \ell}, p_{T, \gamma \gamma}$ and $M_{T}$ distributions for the $1 \ell$ category for $m_{H}=400$ and $600 \mathrm{GeV}$ with backgrounds. Here the heavy Higgs boson is searched for in the $\gamma \gamma W W^{*}$ channel. The distributions are shown after imposing the basic trigger cuts.

with the usual definitions for the variables. Some of the variables of interest are plotted in figure 26. The background yields after the BDT cut are tabulated in table 14 whereas the upper limit on $\sigma(p p \rightarrow H \rightarrow h h)$ as a function of heavy Higgs mass is shown in figure 27. The 95\% CL upper limit for the leptonic scenario is stronger than its semileptonic counterpart in the heavy Higgs mass range of $600 \mathrm{GeV}$ and $1 \mathrm{TeV}$. The upper limit varies in between $109.80 \mathrm{fb}$ and $56.30 \mathrm{fb}$ (110.24 fb and $56.30 \mathrm{fb}$ with $5 \%$ systematic uncertainty) at $95 \%$ CL, in the aforementioned range. 


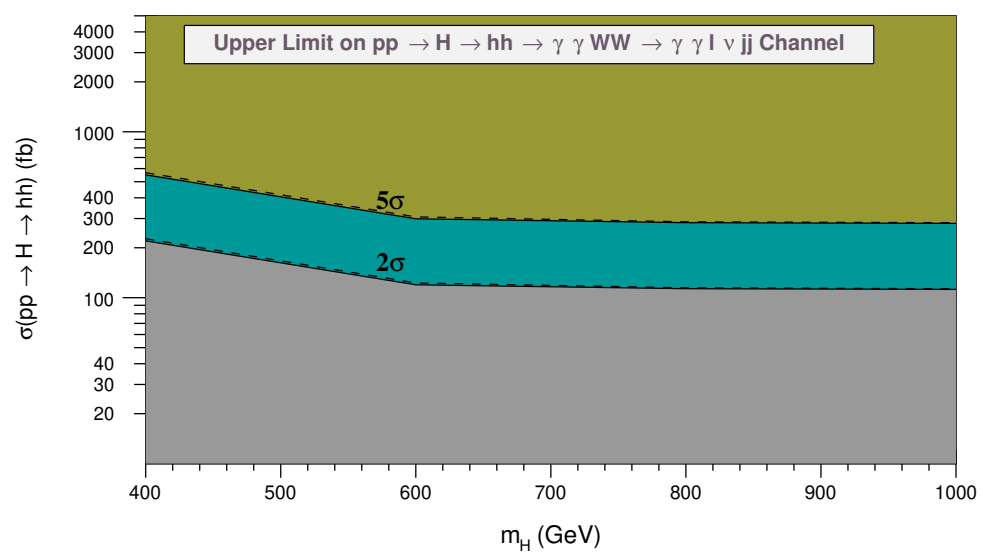

Figure 25. Upper limit on $\sigma(p p \rightarrow H \rightarrow h h)(\mathrm{fb})$ as a function of $m_{H}(\mathrm{GeV})$ for the $\gamma \gamma 1 \ell 2 j+\mathbb{E}_{T}$ channel. The solid (dashed) lines show the $2 \sigma-5 \sigma$ band on taking $0 \%$ (5\%) systematic uncertainties.

\begin{tabular}{|c|c|c|}
\hline (a) & Process & Events \\
\hline & $t \bar{t} h$ & 4.78 \\
& $Z h+$ jets & 1.03 \\
& $p p \rightarrow h h$ & 0.74 \\
Background & $\ell \ell \gamma \gamma+$ jets & 2.44 \\
\cline { 2 - 3 } & Total & 8.99 \\
\hline
\end{tabular}

\begin{tabular}{|c|c|c|}
\hline (b) & Process & Events \\
\hline \multirow{4}{*}{ Background } & $t \bar{t} h$ & 0.89 \\
& $Z h+$ jets & 0.56 \\
& $p p \rightarrow h h$ & 0.37 \\
& $\ell \ell \gamma \gamma+$ jets & 0.64 \\
\cline { 2 - 3 } & Total & 2.46 \\
\hline
\end{tabular}

\begin{tabular}{|c|c|c|}
\hline (c) & Process & Events \\
\hline \multirow{4}{*}{ Background } & $t \bar{t} h$ & 0.26 \\
& $Z h+$ jets & 0.21 \\
& $p p \rightarrow h h$ & 0.12 \\
& $\ell \ell \gamma \gamma+$ jets & 0.24 \\
\cline { 2 - 3 } & Total & 0.83 \\
\hline
\end{tabular}

\begin{tabular}{|c|c|c|}
\hline$(\mathrm{d})$ & Process & Events \\
\hline \multirow{5}{*}{ Background } & $t \bar{t} h$ & 0.14 \\
& $Z h+$ jets & 0.37 \\
& $p p \rightarrow h h$ & 0.05 \\
& $\ell \ell \gamma \gamma+$ jets & 0.14 \\
\cline { 2 - 3 } & Total & 0.70 \\
\hline
\end{tabular}

Table 14. Respective background yields for the $\gamma \gamma 2 \ell+\mathbb{E}_{T}$ channel after the BDT analyses optimised for $m_{H}=(a) 400 \mathrm{GeV},(b) 600 \mathrm{GeV},(c) 800 \mathrm{GeV}$ and $(d) 1 \mathrm{TeV}$. The various perturbative orders for the backgrounds are the same as in table 13.

\subsection{Summarising the $H \rightarrow h h$ channel}

Having studied five different channels with more than one sub-processes in three instances, we summarise the results in this subsection. The 95\% CL upper limits on $\sigma(p p \rightarrow H \rightarrow h h)$ for all these channels is shown in figure 28. We find that the strongest limits come from the $b \bar{b} \gamma \gamma$ and $4 b$ channels. The $b \bar{b} \gamma \gamma$ is strongest up to $m_{H} \sim 600 \mathrm{GeV}$. From $600 \mathrm{GeV}$ onward, the $4 b$ channel is more constraining owing to its larger cross-section. The present limits on $\sigma(p p \rightarrow H \rightarrow h h)$ from the $13 \mathrm{TeV}$ analysis vary between $\sim 970 \mathrm{fb}(300 \mathrm{GeV})$ and $\sim 225 \mathrm{fb}(600 \mathrm{GeV})$ from the $b \bar{b} \gamma \gamma$ channel [138] and between $\sim 70 \mathrm{fb}(800 \mathrm{GeV})$ and $\sim 25 \mathrm{fb}(1 \mathrm{TeV})$ from the $4 b$ analysis [135]. Our projected limits vary between $79.03 \mathrm{fb}$ $(300 \mathrm{GeV})$ and $14.10 \mathrm{fb}(600 \mathrm{GeV})$ from the $b \bar{b} \gamma \gamma$ channel and between $5.36 \mathrm{fb}(800 \mathrm{GeV})$ and $2.51 \mathrm{fb}(1 \mathrm{TeV})$ from the $4 b$ channel. We find an order of magnitude improvement in the sensitivity. We must note in passing that from the results obtained in ref. [142], the 

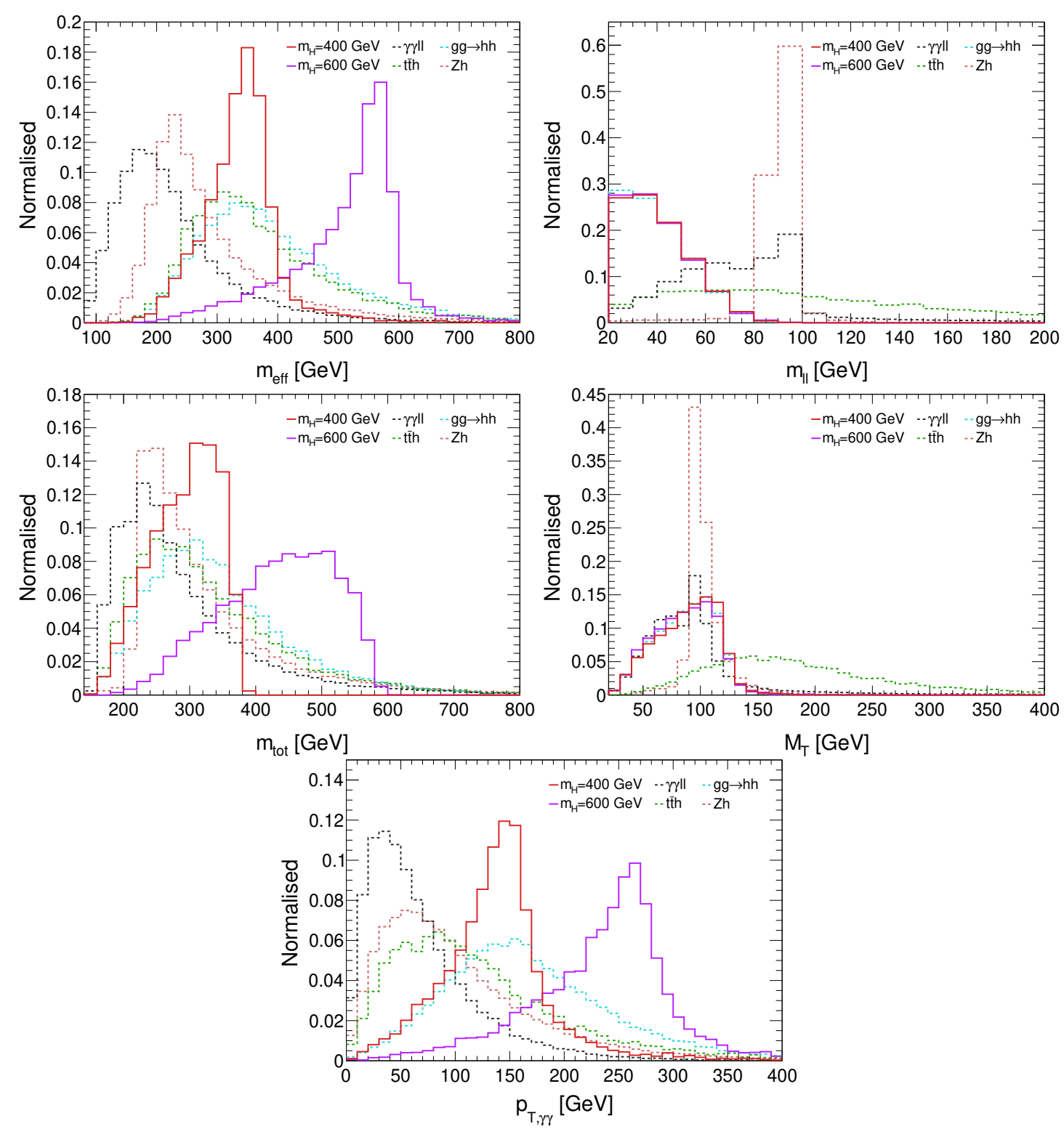

Figure 26. The $m_{\mathrm{eff}}, m_{\ell \ell}, m_{\mathrm{tot}}, M_{T}$ and $p_{T, \gamma \gamma}$ distributions for the $2 \ell$ category for $m_{H}=400$ and $600 \mathrm{GeV}$ with backgrounds. Here the heavy Higgs boson is searched for in the $\gamma \gamma W W^{*}$ channel. The distributions are shown after imposing the basic trigger cuts.

$H \rightarrow h h \rightarrow 4 W$ category does not show much promise even at the HL-LHC, owing to very small signal yields in all categories. The maximum sensitivity is expected in the $e \mu$ 2-lepton category with 5 expected events.

\section{$3 \quad$ The $p p \rightarrow H \rightarrow t \bar{t}$ channel}

After having studied the $H \rightarrow h h$ in multifarious channels in detail, we now turn our attention to a heavy scalar (or pseudoscalar) resonance being produced predominantly by gluon fusion and decaying to a pair of top quarks (figure 29). This channel has already 


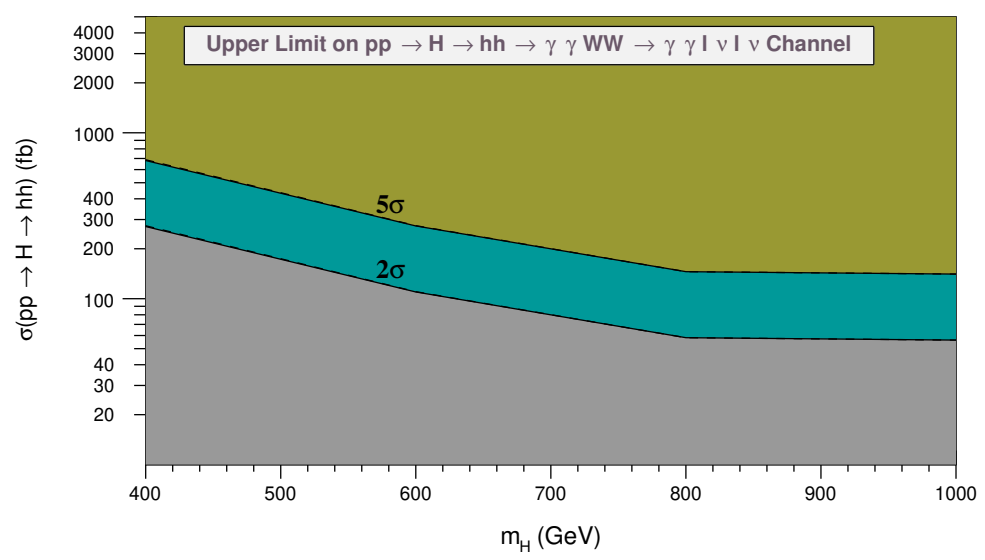

Figure 27. Upper limit on $\sigma(p p \rightarrow H \rightarrow h h)(\mathrm{fb})$ as a function of $m_{H}(\mathrm{GeV})$ for the $\gamma \gamma 2 \ell+\mathbb{E}_{T}$ channel. The solid (dashed) lines show the $2 \sigma-5 \sigma$ band on taking $0 \%$ (5\%) systematic uncertainties.
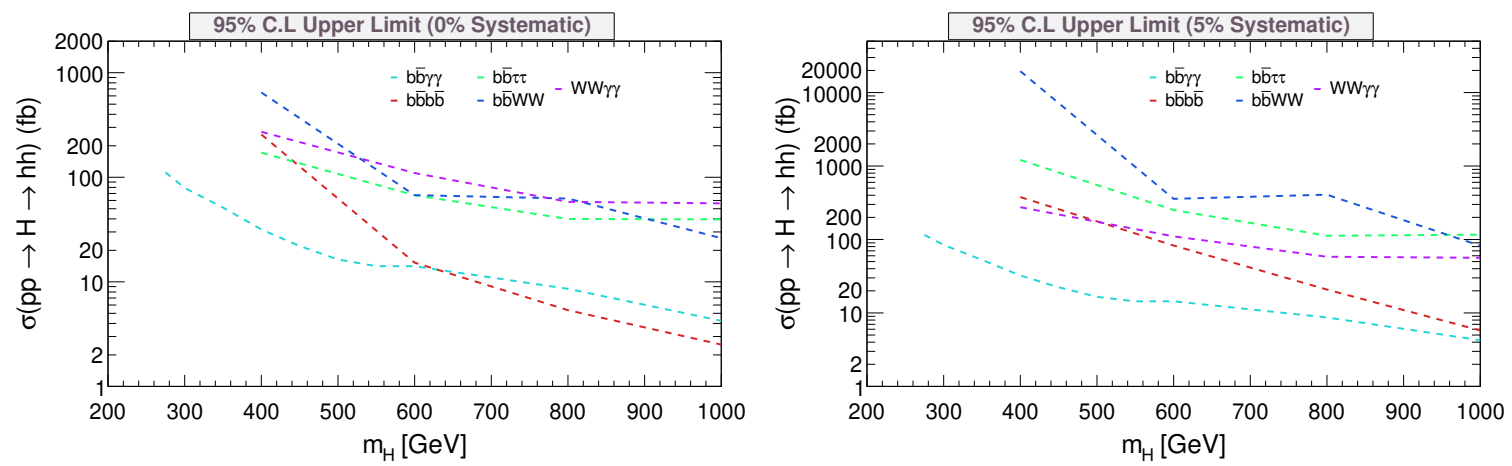

Figure 28. 95\% CL upper limit on $\sigma(p p \rightarrow H \rightarrow h h)$ (fb) as a function of $m_{H}(\mathrm{GeV})$ for the $b \bar{b} \gamma \gamma, b \bar{b} b \bar{b}, b \bar{b} \tau^{+} \tau^{-}, b \bar{b} W W^{*}(2 \ell)$ and $W W^{*} \gamma \gamma(2 \ell)$ channels without systematic uncertainty (left) and with $5 \%$ systematic uncertainty (right).

gained some attention in the experimental community [127, 130]. Searches for resonant scalars, pseudoscalars, $Z^{\prime}$-bosons, Kaluza-Klein gluons and Kaluza-Klein gravitons have been performed. The aim of this section is to try and improve upon these existing searches and provide potential reach of the $\sigma(H \rightarrow t \bar{t})$ by studying the fully leptonic and the semileptonic final states. The branching ratio of $t \rightarrow b W$ being close to $100 \%$ makes the channel essentially become a search for $H \rightarrow b \bar{b} W^{+} W^{-}$. However, unlike the $H \rightarrow h h \rightarrow b \bar{b} W W^{*}$ channel studied in subsection 2.4, where one of the $W$-bosons is off-shell, here both of them are on-shell. This is the first essential difference between the two channels and the reason why one requires a completely different search strategy for the two cases. In the previous section 2, we required $\operatorname{BR}(H \rightarrow h h)=100 \%$. However, in realistic scenarios, if the heavy scalar is produced predominantly via gluon fusion (top/bottom loops), it should also decay to a pair of top quarks (and also bottom quarks) if it is above the $t \bar{t}$ threshold. Similar to the $H \rightarrow h h \rightarrow b \bar{b} W W^{*}$ channel, here also we divide the analysis into two parts, viz., the leptonic and the semi-leptonic channels. We apply the same trigger-level cuts to the various objects as sketched in subsection 2.4. The backgrounds are the same as before. 


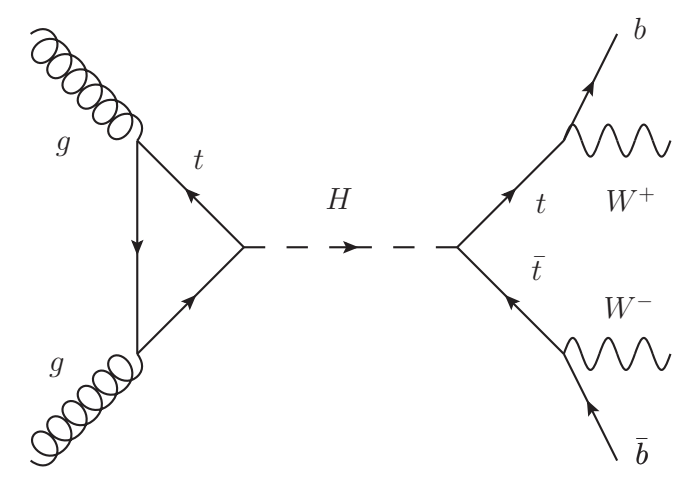

Figure 29. Feynman diagram for the $p p \rightarrow H \rightarrow t \bar{t}$ signal process.

As before, we implement the production and decay of the heavy scalar in the Pythia 6 framework.

\subsection{The leptonic channel}

Like in section 2.4.1, here also we select events with two oppositely charged isolated leptons and two $b$-tagged jets. Without performing a classical cut-based analysis, we optimise our results to obtain the best-possible sensitivity by employing a boosted decision tree analysis. The set of variables which discriminate the signal from the backgrounds are as follows:

$p_{T, b b}, \eta_{b b}, \phi_{b b}, m_{b b}, M_{T}, m_{\mathrm{tot}}, m_{\mathrm{eff}}, \Delta R_{b_{1} \ell_{1}}, p_{T, b_{1}}, p_{T, b_{2}}, p_{T, \ell_{1}}, p_{T, \ell_{2}}$,

where all the variables have their usual meaning as mentioned earlier. We would like to mention here that we also consider the $m_{T 2}$ variable during our analysis. However, this variable is $\sim 80 \%$ correlated with $p_{T, b b}$. Moreover, $m_{T 2}$ has a lower BDT ranking as compared to $p_{T, b b}$. We explicitly checked that adding this correlated variable does not improve our BDT sensitivity. Thus for this analysis, we do not use $m_{T 2}\left(m_{T 2}\right.$ was used in the $b \bar{b} \tau \tau$ (section 2.3) and $b \bar{b} W W^{*}$ (section 2.4) analyses.). The top four discriminatory variables are shown in figure 30 . In table 15 , the number of background events at an integrated luminosity of $3000 \mathrm{fb}^{-1}$, optimised to maximise the sensitivity for various values of $m_{H}$ and after imposing cuts on the BDT observable, are presented. Like in all the other channels, we present the $95 \%$ and $99.7 \%$ upper limit on $\sigma(p p \rightarrow H \rightarrow t \bar{t})$ as a function of $m_{H}$, in figure 31 . We find that the $95 \%$ upper limit on the cross-section lies between $380.43 \mathrm{fb}$ and $135.25 \mathrm{fb}$ (42683.56 fb and $3940.56 \mathrm{fb}$ with $5 \%$ systematic uncertainty) for $m_{H}$ varying between $400 \mathrm{GeV}$ and $1 \mathrm{TeV}$.

\subsection{The semi-leptonic channel}

We end this section by analysing the semi-leptonic final state ensuing from the semi-leptonic decays of $t \bar{t}$. We select events which contain a single isolated lepton, two $b$-tagged jets and at least two light jets after applying the same set of trigger cuts as discussed in section 2.4. 

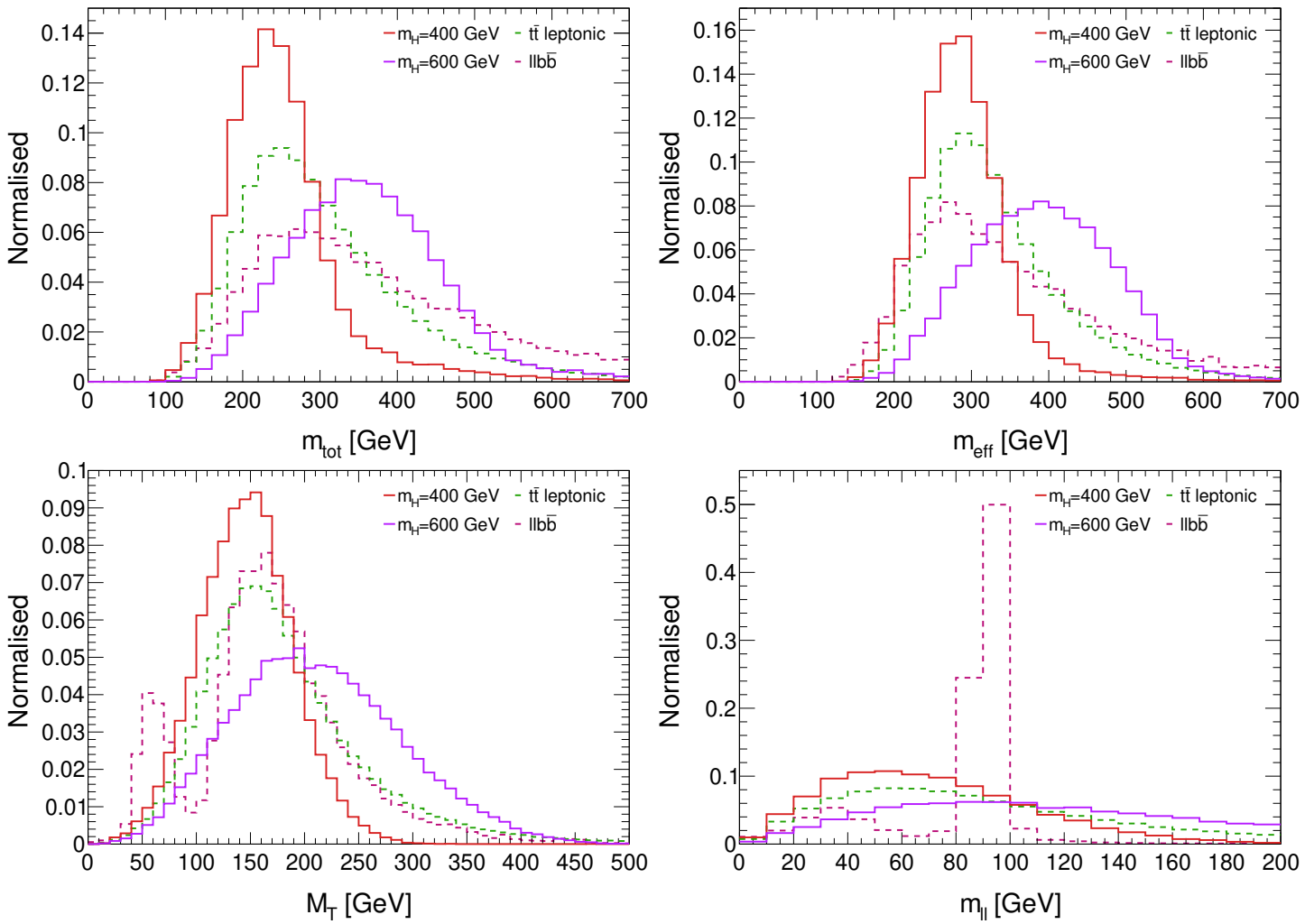

Figure 30. The $m_{\mathrm{tot}}, m_{\mathrm{eff}}, M_{T}$ and $m_{\ell \ell}$ distributions for the $2 \ell$ category for $m_{H}=400$ and $600 \mathrm{GeV}$ with dominant backgrounds. Here the heavy Higgs boson is searched for in the $t \bar{t}$ channel. The distributions are shown before doing the multivariate analysis.

\begin{tabular}{|c|c|c|}
\hline (a) & Process & Events \\
\hline & $t \bar{t}$ lep & 4979032.27 \\
& $t \bar{t} h$ & 6211.98 \\
& $t \bar{t} Z$ & 6769.81 \\
& $t \bar{t} W$ & 4018.71 \\
Background & $p p \rightarrow h h$ & 111.55 \\
& $\ell \ell b \bar{b}$ & 38875.30 \\
\cline { 2 - 3 } & Total & 5035019.62 \\
\hline
\end{tabular}

\begin{tabular}{|c|c|c|}
\hline (b) & Process & Events \\
\hline & $t \bar{t}$ lep & 3520173.16 \\
& $t \bar{t} h$ & 6832.81 \\
& $t \bar{t} Z$ & 10547.04 \\
& $t \bar{t} W$ & 5398.14 \\
Background & $p p \rightarrow h h$ & 73.68 \\
& $\ell \ell b \bar{b}$ & 29580.72 \\
\cline { 2 - 3 } & Total & 3572605.55 \\
\hline
\end{tabular}

\begin{tabular}{|c|c|c|}
\hline$(\mathrm{c})$ & Process & Events \\
\hline & $t \bar{t}$ lep & 712411.06 \\
& $t \bar{t} h$ & 2289.83 \\
& $t \bar{t} Z$ & 4211.79 \\
& $t \bar{t} W$ & 1998.19 \\
Background & $p p \rightarrow h h$ & 32.69 \\
& $\ell \ell b \bar{b}$ & 10697.64 \\
\cline { 2 - 3 } & Total & 731641.20 \\
\hline
\end{tabular}

\begin{tabular}{|c|c|c|}
\hline$(\mathrm{d})$ & Process & Events \\
\hline \multirow{5}{*}{ Background } & $t \bar{t}$ lep & 326174.10 \\
& $t \bar{t} h$ & 1349.87 \\
& $t \bar{t} Z$ & 2866.39 \\
& $t \bar{t} W$ & 1229.00 \\
& $p p \rightarrow h h$ & 23.20 \\
& $\ell \ell b \bar{b}$ & 7492.23 \\
\cline { 2 - 3 } & Total & 339134.79 \\
\hline
\end{tabular}

Table 15. Respective background yields for the $2 \ell+2 b+\mathbb{E}_{T}$ channel after the BDT analyses optimised for $m_{H}=(a) 400 \mathrm{GeV}$, (b) $600 \mathrm{GeV},(c) 800 \mathrm{GeV}$ and $(d) 1 \mathrm{TeV}$. The various orders of the signal and backgrounds are same as in table 11. 


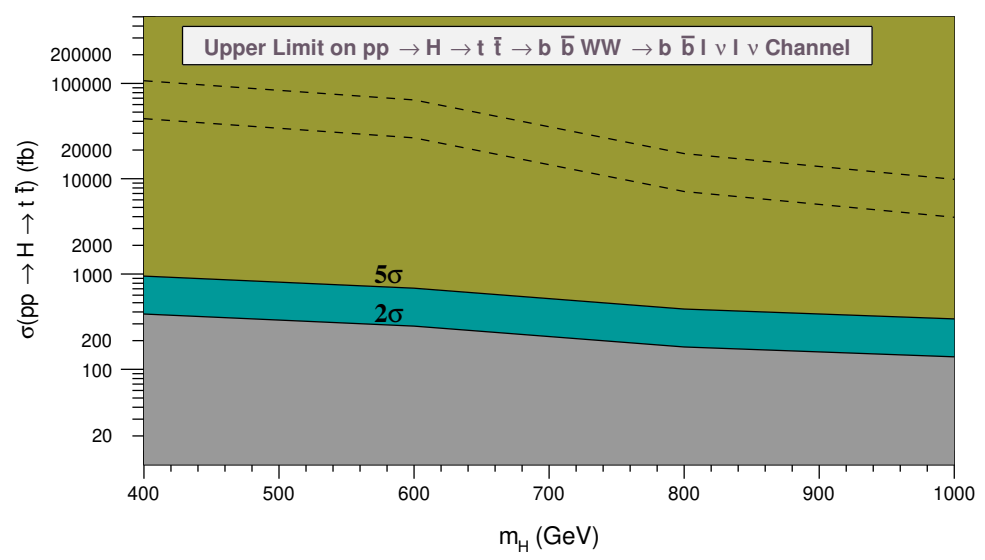

Figure 31. Upper limit on $\sigma(p p \rightarrow H \rightarrow t \bar{t})(\mathrm{fb})$ as a function of $m_{H}(\mathrm{GeV})$ for the $2 \ell+2 b+\mathbb{E}_{T}$ channel. The solid (dashed) lines show the $2 \sigma-5 \sigma$ band on taking $0 \%$ (5\%) systematic uncertainties.
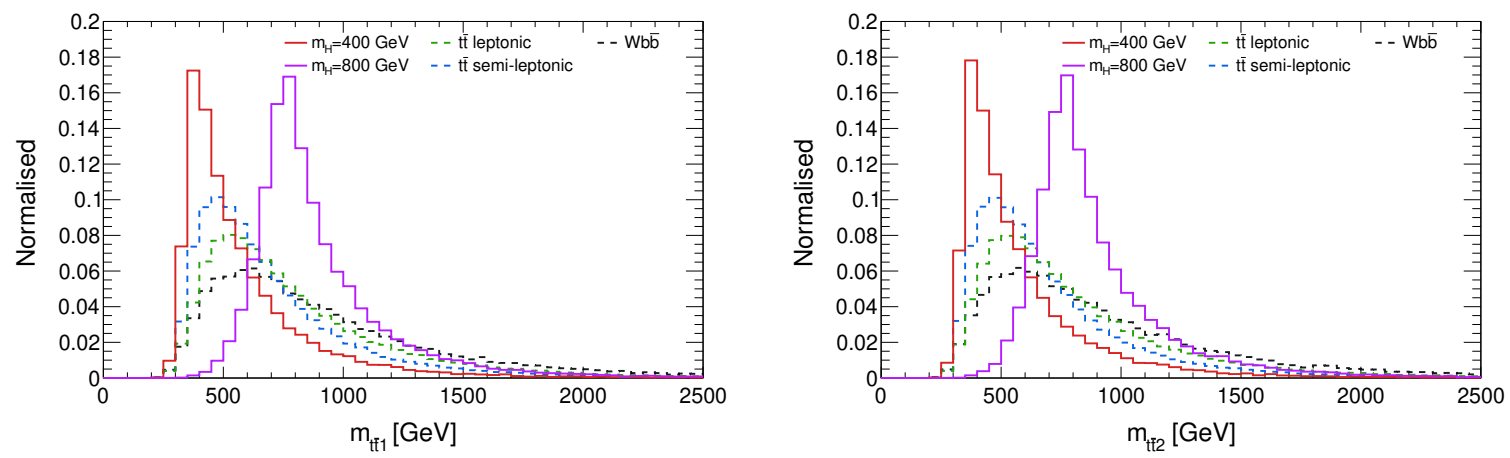

Figure 32. The reconstructed invariant mass of the top-quark pair in the semi-leptonic decay of the $H \rightarrow t \bar{t}$ channel.

Finally, we perform a multivariate analysis with the following set of kinematic variables:

$$
\begin{aligned}
& p_{T, b b}, m_{b b}, \Delta R_{b b}, m_{j j}, m_{\mathrm{eff}}, M_{T}, m_{t 11}, \\
& m_{t 12}, m_{t \bar{t} 1}, m_{t \bar{t} 2}, p_{T, \ell \nu}, \Delta R_{b b, j j}, p_{T, \ell_{1}}, p_{T, j_{1}},
\end{aligned}
$$

where, $m_{t \bar{t} i}$ are the possible combinations for the invariant mass of the heavy Higgs reconstructed from the top pair. The four most sensitive variables are listed in figure 33. The reconstruction procedure is discussed at the beginning of section 2.4. We show the reconstructed $t \bar{t}$ invariant masses in figure 32 . Finally, we summarise the boosted decision tree results in table 16. For heavy Higgs mass ranging between $400 \mathrm{GeV}$ and $1 \mathrm{TeV}$, we show the upper limit on $\sigma(p p \rightarrow H \rightarrow t \bar{t})$ in figure 34. The 95\% CL upper limit varies between $186.57 \mathrm{fb}(39460.45 \mathrm{fb})$ and $32.81 \mathrm{fb}(2021.51 \mathrm{fb})$ for $m_{H}$ varying between $400 \mathrm{GeV}$ and $1 \mathrm{TeV}$ with zero (5\%) systematic uncertainty. The $H \rightarrow t \bar{t}$ channel has a small $S / B$ ratio. Hence, adding systematic uncertainty will drastically change the upper limit on the cross-section. 

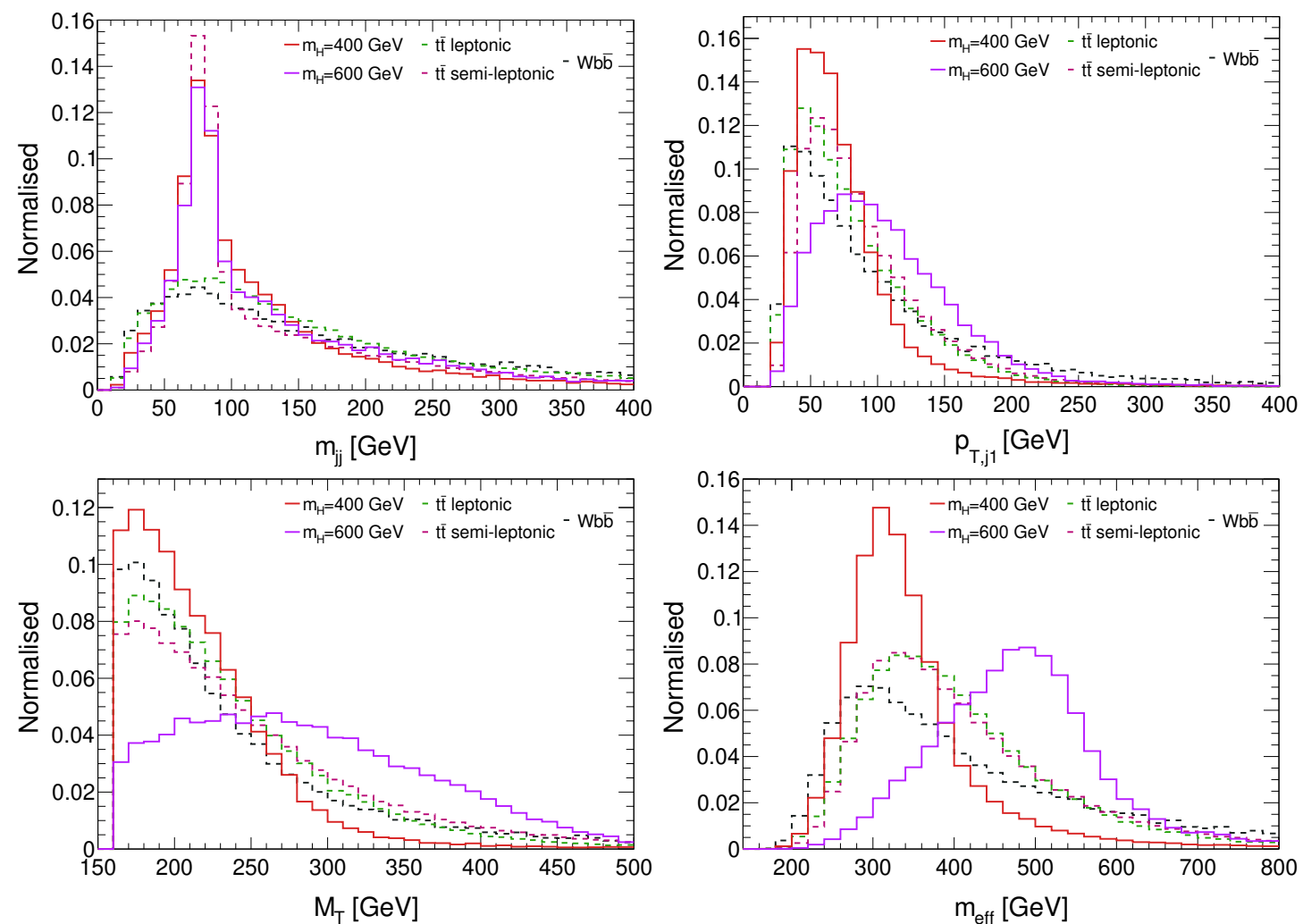

Figure 33. The $m_{j j}, p_{T, j_{1}}, M_{T}$ and $m_{\text {eff }}$ distributions for the semi-leptonic category for $m_{H}=400$ and $600 \mathrm{GeV}$ with dominant backgrounds. Here the heavy Higgs boson is searched for in the $t \bar{t}$ channel. The distributions are shown before doing the multivariate analysis.

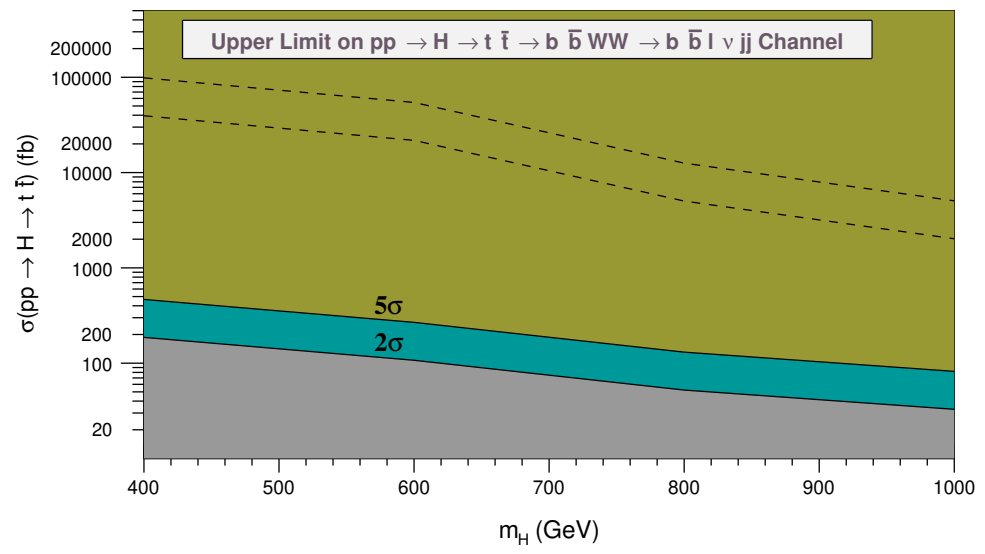

Figure 34. Upper limit on $\sigma(p p \rightarrow H \rightarrow t \bar{t})$ (fb) as a function of $m_{H}(\mathrm{GeV})$ for the $1 \ell 2 j 2 b+\mathbb{E}_{T}$ channel. The solid (dashed) lines show the $2 \sigma-5 \sigma$ band on taking $0 \%$ (5\%) systematic uncertainties. 


\begin{tabular}{|c|c|c|}
\hline (a) & Process & Events \\
\hline & $t \bar{t}$ semi-lep & 15257053.17 \\
& $t \bar{t}$ lep & 2037363.62 \\
& $W b \bar{b}+$ jets & 513737.45 \\
& $t \bar{t} h$ & 23963.91 \\
Background & $t \bar{t} Z$ & 20628.78 \\
& $t \bar{t} W$ & 14852.43 \\
& $p p \rightarrow h h$ & 232.54 \\
& $\ell \ell b \bar{b}$ & 25865.11 \\
\cline { 2 - 3 } & Total & 17893697.01 \\
\hline
\end{tabular}

\begin{tabular}{|c|c|c|}
\hline (b) & Process & Events \\
\hline & $t \bar{t}$ semi-lep & 14297184.84 \\
& $t \bar{t}$ lep & 1620244.31 \\
& $W b \bar{b}+$ jets & 435088.64 \\
& $t \bar{t} h$ & 45147.37 \\
Background & $t \bar{t} Z$ & 42620.52 \\
& $t \bar{t} W$ & 29695.00 \\
& $p p \rightarrow h h$ & 216.70 \\
& $\ell \ell b \bar{b}$ & 13470.49 \\
\cline { 2 - 3 } & Total & 16483667.87 \\
\hline
\end{tabular}

\begin{tabular}{|c|c|c|}
\hline$(\mathrm{c})$ & Process & Events \\
\hline \multirow{7}{*}{ Background } & $t \bar{t}$ semi-lep & 3171586.10 \\
& $t \bar{t}$ lep & 298446.40 \\
& $W b \bar{b}+$ jets & 185875.57 \\
& $t \bar{t} h$ & 15846.33 \\
& $t \bar{t} Z$ & 16523.44 \\
& $t \bar{t} W$ & 12081.76 \\
& $p p \rightarrow h h$ & 66.60 \\
& $\ell \ell b \bar{b}$ & 3244.23 \\
\cline { 2 - 3 } & Total & 3703670.43 \\
\hline
\end{tabular}

\begin{tabular}{|c|c|c|}
\hline$(\mathrm{d})$ & Process & Events \\
\hline \multirow{7}{*}{ Background } & $t \bar{t}$ semi-lep & 1254581.87 \\
& $t \bar{t}$ lep & 115725.81 \\
& $W b \bar{b}+$ jets & 123298.89 \\
& $t \bar{t} h$ & 7762.68 \\
& $t \bar{t} Z$ & 8767.07 \\
& $t \bar{t} W$ & 6720.41 \\
& $p p \rightarrow h h$ & 28.36 \\
& $\ell \ell b \bar{b}$ & 1441.88 \\
\cline { 2 - 3 } & Total & 1518326.97 \\
\hline
\end{tabular}

Table 16. Respective background yields for the $1 \ell 2 j 2 b+\mathbb{E}_{T}$ channel after the BDT analyses optimised for $m_{H}=(a) 400 \mathrm{GeV}$, (b) $600 \mathrm{GeV},(c) 800 \mathrm{GeV}$ and $(d) 1 \mathrm{TeV}$. The various orders of the signal and backgrounds are same as in table 11.

\section{The $(H / A) b \bar{b}$ channel}

Finally, we study the process where the resonant (pseudo)scalar is produced in association with a pair of bottom quarks, viz., $p p \rightarrow(b \bar{b}) H / A$. The need to study this process lies in the fact that one can probe and impose strong limits on the lower part in the $m_{A}-\tan \beta$ plane, as will be discussed in section 5 . The cross-section of the inclusive $(b \bar{b}) H$ process receives contribution from both the 4 -flavour $(4 \mathrm{~F})$ and the 5 -flavour $(5 \mathrm{~F})$ processes. There are two QCD processes (figure 35 ) contributing to the $4 \mathrm{~F}$ scheme at LO where the heavy Higgs is produced in association with two $b$-quarks, one is via the gluon fusion process $(g g \rightarrow b \bar{b} H)$ and the other is via quarks $(q \bar{q} \rightarrow b \bar{b} H)$. The $4 \mathrm{~F}$ inclusive cross-section suffers from large logarithms due to an almost collinear splitting of a gluon into a pair of bottom quarks. This is of the form $\ln \left(\frac{\mu_{F}}{m_{b}}\right)\left(\mu_{F} \equiv\right.$ factorisation scale) and may lead to a breakdown of the perturbative theory. However, these logarithms can be absorbed inside the bottom quark parton distribution function (PDF) by re-summing at all orders in the perturbation theory. This forms the basis of the $5 \mathrm{~F}$ scheme. At leading order (LO), the $5 \mathrm{~F}$ scheme is dominated by the QCD process $b \bar{b} \rightarrow H$ (figure 35 ). However, for scenarios involving $b$-jet in the final state, the processes where the resonant scalar is produced in association with a $b$-quark or a gluon, becomes important, viz., $g b(b \bar{b}) \rightarrow b H(g H)$ (figure 35). Owing to different perturbative expansions, these two schemes give different results when truncated at any finite order. Thus, higher order calculations become important to match these two results. The $4 \mathrm{~F}$ scheme calculation is available up to NLO in QCD [158-161], while the 5F scheme 


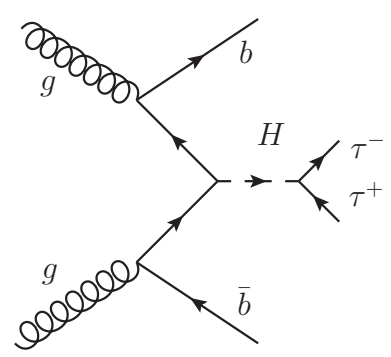

(a)

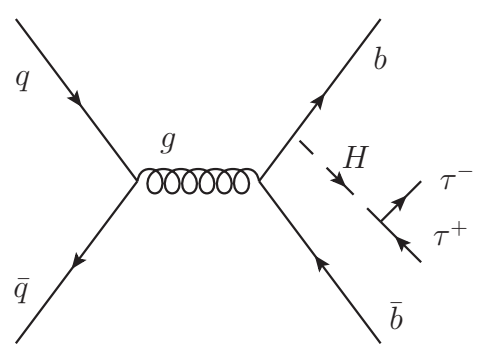

(b)

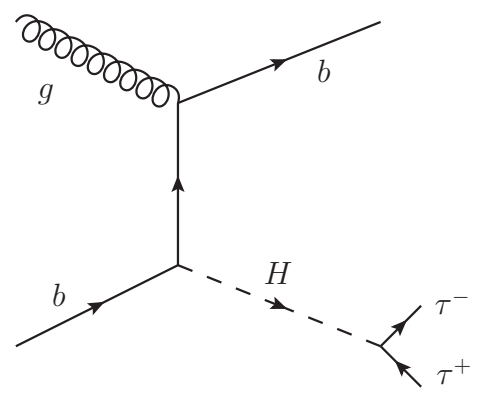

$(d)$

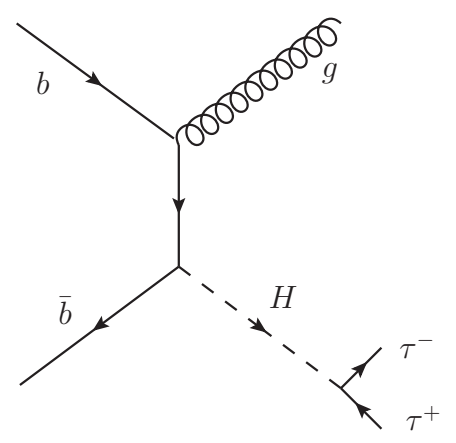

$(e)$

Figure 35. The Feynman diagrams for $4 \mathrm{~F}$ (a) $g g \rightarrow b \bar{b} H$ and (b) $q \bar{q} \rightarrow b \bar{b} H$ process, and $5 \mathrm{~F}$ (c) $b \bar{b} \rightarrow H$ (LO), (d) $g b \rightarrow b H$ and (e) $b \bar{b} \rightarrow g H$ process.

is known up to NNLO accuracy in QCD [162]. Here the LO process in the 4F scheme i.e. $g g \rightarrow b \bar{b} H$ appears at the NNLO order in $5 \mathrm{~F}$ scheme. The resonant scalar production in association with a $b$-quark or gluon i.e. $g b(b \bar{b}) \rightarrow b H(g H)$ has been calculated up to NLO in QCD [163] and electroweak (EW) [164].

It has been argued that with a proper choice of factorisation scale $\sim \frac{m_{H}}{4}$, the inclusive cross-section in the $4 \mathrm{~F}$ and $5 \mathrm{~F}$ schemes agree very well [165-167]. There is a proposed way to combine these two approaches. This is known as the Santander matching [168] scheme. The total inclusive cross-section is obtained by matching the $4 \mathrm{~F}$ and $5 \mathrm{~F}$ scheme numbers in which both these cross-sections are multiplied by their proper weight factors. These weight factors change logarithmically with the heavy scalar mass $\left(m_{H}\right)$ because of logarithmic difference between these two scheme approaches. The matched cross-section is 
computed as follows:

$$
\sigma^{\text {matched }}=\frac{\sigma^{4 \mathrm{FS}}+w \sigma^{5 \mathrm{FS}}}{1+w}
$$

where

$$
w=\ln \frac{m_{H}}{m_{b}}-2
$$

is the weight factor. ${ }^{10}$ The analysis can be subdivided according to the number of $b$-tagged jets. However, we specifically focus on the category with $\geq 1$ b-jets upon following a recent study performed by the ATLAS collaboration [132]. Furthermore, we consider the heavy Higgs decaying to a pair of $\tau$-leptons and we specifically focus on the scenario where both the $\tau$ s decay hadronically. The $H$ and $A$ masses are varied between $200 \mathrm{GeV}$ and $1 \mathrm{TeV}$.

The various backgrounds at play are $Z / \gamma^{*}+$ jets, multijets, $W+$ jets, $V V\left(V=W^{ \pm}, Z\right)$, $t \bar{t}$ and single top. The $Z / \gamma^{*}+$ jets with the $Z$-boson decaying to a pair of leptons $(e, \mu$ and $\tau$ ) is the dominant background for the $\tau_{h} \tau_{\ell}$ category (a category that we will not address in the present work) but also gives significant contribution to the $\tau_{h} \tau_{h}$ category. We simulate this background merged with three additional partons and some specific generation level cuts which are described in appendix A. Similarly, the $W+$ jets is also generated with up to three additional partons and the $W$-boson is then decayed leptonically. In order to include the dominant multijets background in the $\tau_{h} \tau_{h}$ category, we generate an exclusive $b \bar{b} j j$ sample where $j$ includes light quarks and gluon. These light jets can fake hadronically decaying $\tau$ s. Finally, we include the top-quark related backgrounds viz., $t \bar{t}$ and single top. Next, we describe our analysis for the $\geq 1 b$-tagged jets category upon closely following ref. [132].

\subsection{The $\tau_{h} \tau_{h}$ channel: $b$-tag category}

We select events containing at least one $b$-tagged jet with $p_{T}>20 \mathrm{GeV}$ and two $\tau$-tagged jets with $p_{T}>65 \mathrm{GeV} .{ }^{11}$ These two $\tau$-tagged jets must have opposite electric charge (from their track reconstruction). We also veto events having leptons $(e, \mu)$ or $\tau$-tagged jets with $1.37<\left|\eta_{\tau}\right|<1.52$, in the final state. The azimuthal angle separation between the two $\tau$-tagged jets has to fulfil the condition, $|\Delta \phi(\tau, \tau)|>2.7$. The $b$ - and the $\tau$-jets must have an angular separation in the $\eta-\phi$ plane, viz., $\Delta R(b, \tau)>0.2$. Besides, we also impose a minimum bound on the visible invariant mass of the two hadronically decaying $\tau$ leptons to be $m_{\tau \tau}^{\text {vis. }}>50 \mathrm{GeV}$. For the fake $b \bar{b} j j$ background, we demand the two light jets to satisfy the $\tau$ jet configuration during the analysis and we later multiply the event yield with the $j \rightarrow \tau$ fake rate. Similarly, for the $W(\rightarrow \tau \nu$ or $\ell \nu)+$ jets background, we demand at least one extra light jet satisfying the $\tau$ jet requirement on top of the $\tau$ jet ensuing from $W$-boson decay. After imposing the aforementioned cuts, we improve our analysis by optimising over some other kinematic variables viz., the transverse momentum of the hardest $\tau$-tagged jet $\left(p_{T, \tau_{1}}\right)$, sum of the cosine of the azimuthal angle separation between

\footnotetext{
${ }^{10}$ Here, $m_{b}$ is the bottom quark pole mass which enters in the re-summed logarithms.

${ }^{11}$ Before performing this analysis, we validated our setup with the ATLAS analysis at $13 \mathrm{TeV}$. The validation is shown in appendix $\mathrm{B}$.
} 

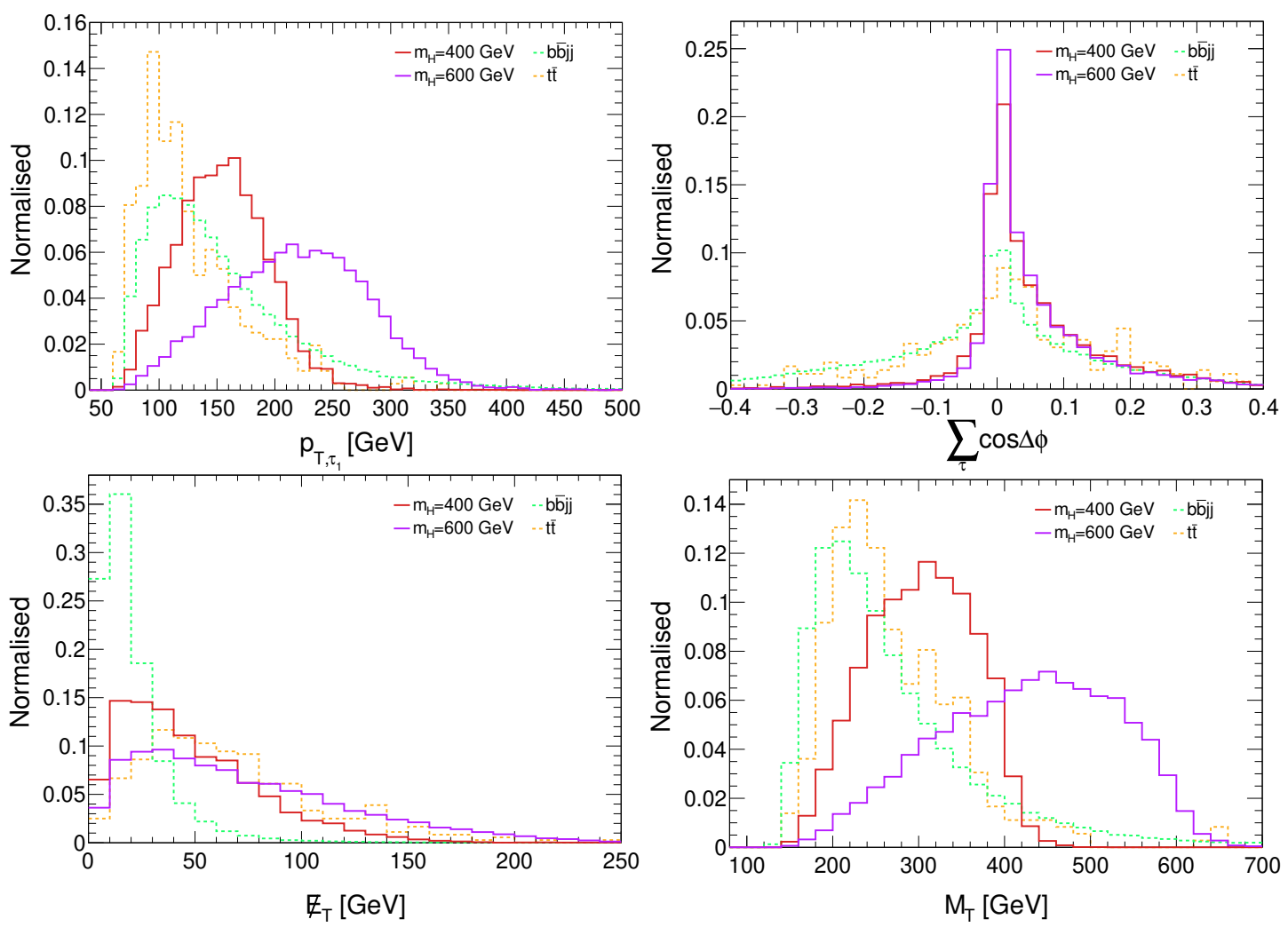

Figure 36. The $p_{T, \tau_{1}}, \sum_{\tau_{1,2}} \cos \Delta \phi, \mathbb{E}_{T}$ and $M_{T}$ distributions for the $b \bar{b} \tau_{h} \tau_{h}$ category for $m_{H}=400$ and $600 \mathrm{GeV}$ with dominant backgrounds. Here the heavy Higgs boson is searched for in the $b \bar{b} H$ channel. The distributions are shown before the optimisation analysis.

the $\tau$-jets and $\mathbb{E}_{T}\left(\sum_{\tau_{1,2}} \cos \Delta \phi\right)$ and the transverse mass of the total system which is defined below,

$$
M_{T}=\sqrt{\left(p_{T, \tau_{1}}+p_{T, \tau_{2}}+\mathscr{E}_{T}\right)^{2}-\left(\vec{p}_{T, \tau_{1}}+\vec{p}_{T, \tau_{2}}+\overrightarrow{\mathbb{E}_{T}}\right)^{2}}
$$

where the symbols have their usual meanings. These four kinematic variables are shown in figure 36. The optimised cuts along with the signal efficiencies and the background yields for each benchmark point are shown in table 17. We perform our analysis upon considering both the $4 \mathrm{~F}$ and $5 \mathrm{~F}$ signal samples separately. Finally, we add them by multiplying these cross-sections with the aforementioned weight factor in order to obtain the upper limit on the matched $b \bar{b} H$ production cross section. We show the $95 \%$ and $99.7 \%$ exclusion for $\sigma(p p \rightarrow b \bar{b} H) \times \mathrm{BR}\left(H \rightarrow \tau_{h} \tau_{h}\right)$ in figure 37. The $95 \%$ upper limit varies between $22.16 \mathrm{fb}$ and $3.68 \mathrm{fb}$ (within $[146.91,3.70] \mathrm{fb}$ with $5 \%$ systematic uncertainty) for $m_{H}$ varying between $300 \mathrm{GeV}$ and $500 \mathrm{GeV}$. This is close to an order of magnitude improvement over the existing bounds at $13 \mathrm{TeV}$ [132]. The effects of systematic uncertainties for this $b \bar{b} H$ channel become negligible for $m_{H}>400 \mathrm{GeV}$. However, for lower masses, the backgrounds are larger and thus the inclusion of uncertainties weaken the limits. 


\begin{tabular}{|c|c|c|c|c|c|c|}
\hline \multirow{2}{*}{$\begin{array}{c}\text { Heavy Higgs mass, } \\
m_{H}(\mathrm{GeV})\end{array}$} & \multicolumn{4}{|c|}{ Optimised cuts $(\mathrm{GeV})$} & \multicolumn{2}{c|}{ After all cuts } \\
\cline { 2 - 7 } & $p_{T, \tau_{1}}>$ & $\sum_{\tau} \cos \Delta \phi>$ & $M_{T}$ & $\mathbb{E}_{T}>$ & Signal Efficiency $\left(\times 10^{-4}\right)$ & ${\text { Background yield at } 3000 \mathrm{fb}^{-1}}$ \\
\hline 200 & 70 & -0.10 & {$[80,200]$} & 0 & 7.94 & 3725.90 \\
\hline 300 & 75 & -0.06 & {$[160,320]$} & 0 & 49.40 & 17172.83 \\
\hline 400 & 180 & -0.04 & {$[380,400]$} & 80 & 1.39 & 2.22 \\
\hline 500 & 180 & -0.02 & {$[380,420]$} & 80 & 5.14 & 5.16 \\
\hline 600 & 240 & -0.10 & {$[500,580]$} & 140 & 5.38 & 0.62 \\
\hline 800 & 260 & 0.00 & {$[400,840]$} & 220 & 7.91 & 0.62 \\
\hline 1000 & 260 & -0.02 & {$[780,1020]$} & 200 & 23.03 & 0.36 \\
\hline
\end{tabular}

Table 17. The details of final optimised cuts with signal efficiency and background yields after all the applied cuts.

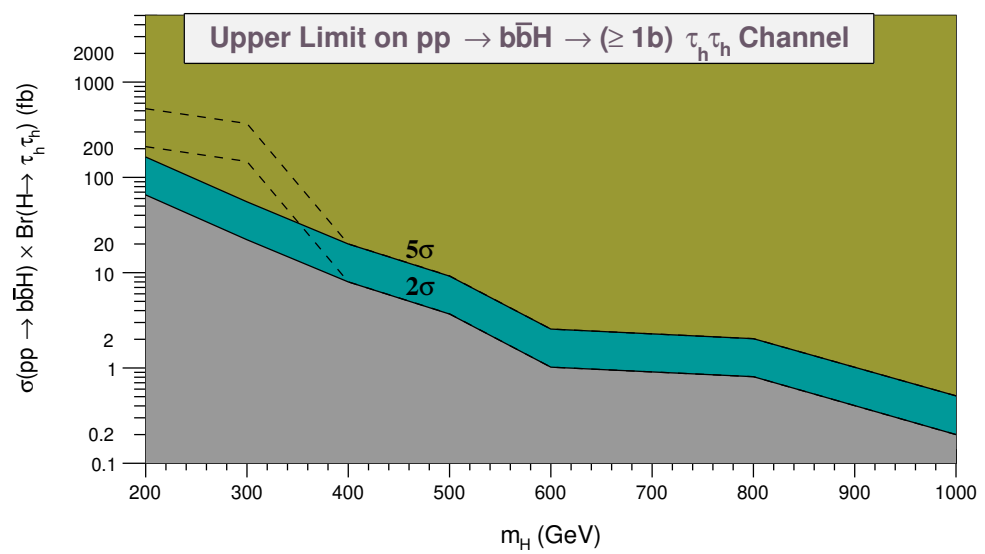

Figure 37. Upper limit on $\sigma\left(p p \rightarrow b \bar{b} H \rightarrow b \bar{b} \tau^{+} \tau^{-}\right)(\mathrm{fb})$ as a function of $m_{H}(\mathrm{GeV})$ for the $\geq 1 b+2 \tau_{h}$ channel. The solid (dashed) lines show the $2 \sigma-5 \sigma$ band on taking $0 \%$ (5\%) systematic uncertainties.

\section{The future of the pMSSM parameter space}

The Higgs sector in the MSSM comprises two Higgs doublets which give rise to five massive Higgs states after the electroweak symmetry breaking. The Higgs spectrum is thus composed of two $C P$-even scalars, $h$ and $H$, one $C P$-odd scalar, $A$, and two charged scalars, $H^{ \pm}$(detailed studies on the Higgs sector of MSSM can be found in refs. [36, 169]). In addition to the extended Higgs sector, the SUSY particle spectrum boasts a multitude of particles, viz., the sleptons, squarks, gluinos and electroweakinos. A majority of direct searches at the LHC have excluded stops and gluinos below the TeV scale (refs. [170-178] show some such limits in various supersymmetric interpretations). This more or less nullifies the prospect of observing these particles unless the luminosity is enhanced significantly. The electroweakino sector has also been probed in numerous studies [179-184] and bounds have been obtained on their masses within simplified scenarios [185-194]. In many of these studies, the electroweakino masses are excluded from between a few hundred GeVs to about half a $\mathrm{TeV}$ and are comparatively weakly coupled compared to the gluinos and stops. Within a generic SUSY parameter space without any correlation between the choice 
of the electroweakino mass parameters, these bounds can become considerably weaker. The ATLAS and CMS collaborations have also performed several studies to search for resonant Higgs through their decay into SM final states [59, 123, 132, 134, 136, 195-199]. However, none of these searches could find any significant excess over the SM expectations and thus only imposed upper limits on the production cross-section of the heavy Higgs bosons times their branching ratio into various SM final states. In this section, we present a brief discussion on the relevant constraints and discuss the parameter scan for the $14 \mathrm{TeV}$ HL-LHC. We follow this up with an analysis to capture the present status of the MSSM parameter space in light of the latest results from the Run-II data $\left(13 \mathrm{TeV}, 36 \mathrm{fb}^{-1}\right)$ of LHC. Finally, we study the implications of the projected heavy Higgs direct search limits derived in sections 2, 3 and 4, on the MSSM parameter space.

The initial constraint on the parameter space ensues from the allowed mass of the $125 \mathrm{GeV}$ SM-like Higgs boson. A combined measurement by the ATLAS and CMS collaborations constrains $m_{h}$ within the range $[124.4,125.8] \mathrm{GeV}$ at $3 \sigma$. It is to be duly noted that in the context of MSSM, the available calculation of the Higgs mass is not exact. Thus, in order to correctly account for the existing uncertainties, we allow a window of $\pm 3 \mathrm{GeV}$ about $125 \mathrm{GeV}$ and restrict the light Higgs mass in our parameter space to lie within $[122$, 128] GeV. Furthermore, both collaborations have performed numerous measurements on the coupling strengths of the SM-like Higgs bosons. These results are presented through bounds on the signal strength variable $\left(\mu^{i f}\right)$ which is defined as follows:

$$
\mu_{i f}=\frac{\sigma_{i} \times \mathrm{BR}_{f}}{\sigma_{i}^{\mathrm{SM}} \times \mathrm{BR}_{f}^{\mathrm{SM}}}
$$

where, $\sigma_{i}$ represents the MSSM (or any specific model in question) Higgs production crosssection in the $i^{t h}$ production mode $(i=g g F, V B F, t \bar{t} h$ or $V h)$ at the LHC and $\sigma_{i}^{\mathrm{SM}}$ denotes the corresponding SM cross-section. $\mathrm{BR}_{f}$ corresponds to the branching fraction of the SM-like Higgs into a particular SM final state $(f=W W, Z Z, b \bar{b}, \gamma \gamma, \tau \tau)$ and $\mathrm{BR}_{f}^{\mathrm{SM}}$ is the corresponding SM value. We apply all these constraints over our parameter space by demanding that all our signal strengths simultaneously lie within $2 \sigma$ of their experimental counterparts. The latest Higgs signal strengths $\left(13 \mathrm{TeV}, 36 \mathrm{fb}^{-1}\right)$ measured by both the CMS and ATLAS collaborations are listed in table 18.

Additionally, the flavour physics bounds also potentially constrain the MSSM parameter space, as shown in [39]. In this regard, the bounds on the branching fraction of rare $B$-decay processes: $B \rightarrow X_{s} \gamma, B_{s} \rightarrow \mu^{+} \mu^{-}, B^{+} \rightarrow \tau^{+} \nu_{\tau}$, are among the most sensitive probes of new physics searches. As shown in [39], constraints from $B \rightarrow X_{s} \gamma$ disfavours the low $M_{A}$ regime while bounds on $\operatorname{Br}\left(B_{s} \rightarrow \mu^{+} \mu^{-}\right)$constrains the low $M_{A}$ and high $\tan \beta$ regions. The low $M_{A}$ and high $\tan \beta$ region gets further constrained by $B^{+} \rightarrow \tau^{+} \nu_{\tau}$. On the other hand, the current limits from direct heavy Higgs searches in $b \bar{b} H / A, H / A \rightarrow \tau^{+} \tau^{-}$, imposes much stringent constraint in the $M_{A} \gtrsim 300 \mathrm{GeV}$ region and excludes $\tan \beta$ up to $\sim 18$ for $M_{A} \sim 1 \mathrm{TeV}$ (a detailed discussion concerning this can be found in [39]). Consequently, within the scope of this section where our major emphasis is on exploring the future reach of direct heavy Higgs searches at the HL-LHC, we do not consider the implications from flavour physics bounds and impose only the light Higgs mass constraint 


\begin{tabular}{|c|c|c|c|c|}
\hline \multirow{2}{*}{$\begin{array}{l}\text { Production } \\
\text { mode }\end{array}$} & \multicolumn{2}{|c|}{ CMS [200] } & \multicolumn{2}{|c|}{ ATLAS } \\
\hline & $\begin{array}{c}\text { Decay } \\
\text { channel }\end{array}$ & $\begin{array}{l}\text { Best fit } \\
\text { value }\end{array}$ & $\begin{array}{c}\text { Decay } \\
\text { channel }\end{array}$ & $\begin{array}{l}\text { Best fit } \\
\text { value }\end{array}$ \\
\hline \multirow{5}{*}{$g g h$} & $b \bar{b}$ & $2.51_{-2.01}^{+2.43}$ & $W W$ & $1.21_{-0.21}^{+0.22}[201]$ \\
\hline & $\tau^{+} \tau^{-}$ & $1.05_{-0.47}^{+0.53}$ & $Z Z$ & $1.17_{-0.50}^{+0.41}[202]$ \\
\hline & $W W^{*}$ & $1.35_{-0.19}^{+0.21}$ & $\gamma \gamma$ & $0.81_{-0.18}^{+0.19}[203]$ \\
\hline & $Z Z^{*}$ & $1.22_{-0.21}^{+0.23}$ & & \\
\hline & $\gamma \gamma$ & $1.16_{-0.18}^{+0.21}$ & & \\
\hline \multirow{4}{*}{$V B F$} & $\tau \tau$ & $1.12_{-0.43}^{+0.45}$ & $b \bar{b}$ & $3.00_{-1.60}^{+1.70}[204]$ \\
\hline & $W W^{*}$ & $0.28_{-0.60}^{+0.64}$ & $W W$ & $0.62_{-0.36}^{+0.37}[201]$ \\
\hline & $Z Z^{*}$ & $-0.09_{-0.76}^{+1.02}$ & $\gamma \gamma$ & $2.00_{-0.50}^{+0.60}[203]$ \\
\hline & $\gamma \gamma$ & $0.67_{-0.46}^{+0.59}$ & & \\
\hline \multirow{4}{*}{$W h$} & $b \bar{b}$ & $1.73_{-0.68}^{+0.70}$ & $b \bar{b}$ & $1.08_{-0.43}^{+0.47}[13]$ \\
\hline & $W W^{*}$ & $3.91_{-2.01}^{+2.26}$ & $b \bar{b}$ & $1.21_{-0.42}^{+0.45}[205]$ \\
\hline & $Z Z^{*}$ & $0.00_{-0.00}^{+2.33}$ & & \\
\hline & $\gamma \gamma$ & $3.76_{-1.35}^{+1.48}$ & & \\
\hline \multirow{4}{*}{$Z h$} & $b \bar{b}$ & $0.99_{-0.45}^{+0.47}$ & $b \bar{b}$ & $1.20_{-0.31}^{+0.33}[13]$ \\
\hline & $W W^{*}$ & $0.96_{-1.46}^{+1.81}$ & $b \bar{b}$ & $0.69_{-0.33}^{+0.35}[205]$ \\
\hline & $Z Z^{*}$ & $0.00_{-0.00}^{+4.26}$ & & \\
\hline & $\gamma \gamma$ & $0.00_{-0.00}^{+1.14}$ & & \\
\hline \multirow{5}{*}{$t \bar{t} h$} & $b \bar{b}$ & $0.91_{-0.43}^{+0.45}$ & $\frac{\sigma_{t \bar{t} h}}{\sigma_{t \bar{t} h_{\mathrm{SM}}}}$ & $0.84_{-0.61}^{+0.64}[206]$ \\
\hline & $\tau^{+} \tau^{-}$ & $0.23_{-0.88}^{+1.03}$ & $b \bar{b}$ & $0.80_{-0.60}^{+0.60}[207]$ \\
\hline & $W W^{*}$ & $1.60_{-0.59}^{+0.65}$ & $\gamma \gamma$ & $0.60_{-0.60}^{+0.70}[207]$ \\
\hline & $Z Z^{*}$ & $0.00_{-0.00}^{+1.50}$ & & \\
\hline & $\gamma \gamma$ & $2.18_{-0.75}^{+0.88}$ & & \\
\hline
\end{tabular}

Table 18. Best-fit value of signal strength variables, along with the associated errors, derived by ATLAS and CMS using LHC Run-II data, which have been imposed on the parameter space region.

and Higgs signal strength constraints in order to obtain the allowed parameter space region relevant for studying the current and future reach of direct heavy Higgs searches on the MSSM parameter space.

In order to evaluate the current allowed region in the parameter space of the phenomenological MSSM (pMSSM), we perform a random scan over a wide range of pMSSM input parameters, as described below. The parameters relevant to our study are the pseudoscalar mass variable $\left(m_{A}\right)$, ratio of the vacuum expectation values of the two Higgs doublets $(\tan \beta)$, the third generation soft squark mass parameters $\left(M_{\tilde{Q}_{3}}, M_{\tilde{u}_{3}}, M_{\tilde{d}_{3}}\right)$, the trilinear coupling of the stop $\left(A_{t}\right)$ and sbottom $\left(A_{b}\right)$ and the gluino mass parameter $\left(M_{3}\right)$. These 


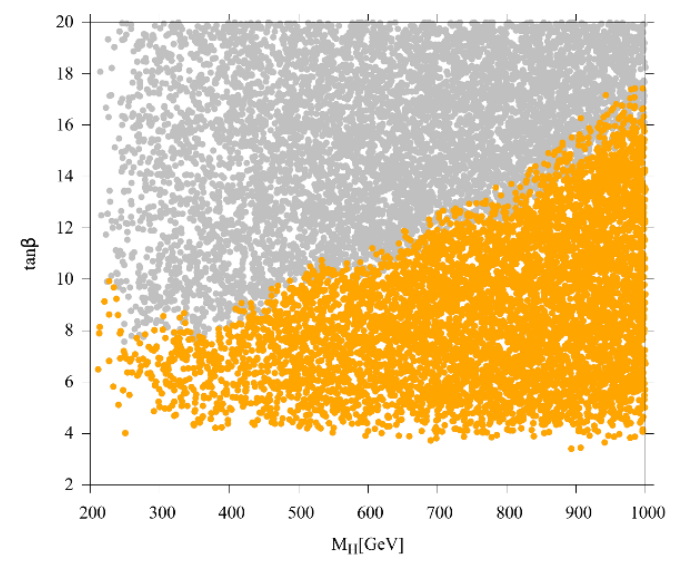

Figure 38. Scatter plot in the $m_{A}-\tan \beta$ plane showing the current status of the pMSSM parameter space. All parameter space points satisfy constraints from the Higgs mass measurement and the Higgs signal strengths. The grey coloured points are excluded by the latest direct search limits from $\sigma_{b \bar{b} H / A} \times B r(H / A \rightarrow \tau \tau)$ derived by CMS and ATLAS using the Run-II dataset with an integrated luminosity of $\sim 36 \mathrm{fb}^{-1}$.

parameters are varied in the following range:

$$
\begin{aligned}
& 1<\tan \beta<60, \quad 200 \mathrm{GeV}<m_{A}<1 \mathrm{TeV}, \quad 1 \mathrm{TeV}<M_{3}<10 \mathrm{TeV} \\
& 1 \mathrm{TeV}<M_{\tilde{Q}_{3}, \tilde{u}_{3}, \tilde{d}_{3}}<20 \mathrm{TeV}, \quad-10 \mathrm{TeV}<A_{t, b}<10 \mathrm{TeV} \\
& 1 \mathrm{TeV}<M_{\tilde{Q}_{1}, \tilde{u}_{1}, \tilde{d}_{1}}<20 \mathrm{TeV}, \quad M_{\tilde{Q}_{2}}=M_{\tilde{Q}_{1}}, M_{\tilde{u}_{2}}=M_{\tilde{u}_{1}}, M_{\tilde{d}_{2}}=M_{\tilde{d}_{1}}
\end{aligned}
$$

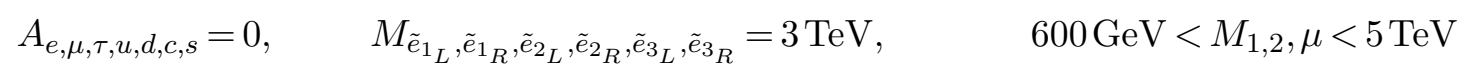

The bino, wino and higgsino mass parameters, viz., $M_{1}, M_{2}$ and $\mu$ respectively, are varied from $600 \mathrm{GeV}$ in order to prevent the heavier Higgs bosons from having any decays to the electroweakinos. This choice ensures only SM final states for the heavy Higgs boson decays. The second generation soft squark mass parameters $\left(M_{\tilde{Q}_{2}, \tilde{u}_{2}, \tilde{d}_{2}}\right)$ are taken to be equal to their corresponding first generation counterparts $\left(M_{\tilde{Q}_{1}, \tilde{u}_{1}, \tilde{d}_{1}}\right)$. The slepton mass parameters $\left(\tilde{e}_{1_{L}, 1_{R}, 2_{L}, 2_{R}, 3_{L}, 3_{R}}\right)$ are fixed at $3 \mathrm{TeV}$ while the trilinear couplings of the first and the second generation squarks $\left(A_{u, d, c, s}\right)$ and all three generations of sleptons $\left(A_{e, \mu, \tau}\right)$ are taken to be zero.

The particle spectra and the branching fractions of the SM and SUSY particles are obtained using FeynHiggs [208]. We consider only those parameter points which satisfy the light Higgs mass constraint defined above. Furthermore, we allow only those points which lie within $2 \sigma$ uncertainty of each of the signal strength variables listed in table 18 . The parameter space points which are allowed by the aforementioned light Higgs mass constraint and the Higgs signal strength constraints are referred to as the allowed parameter space points in the remainder of this section and are shown in grey in figure 38.

The ATLAS and CMS collaborations have also performed numerous searches for the heavy Higgs bosons through their decay into the SM final states, however, none of these 
searches have been able to observe any significant excess over the SM expectation. Consequently, upper limits have been set on the production cross-section of the heavy Higgs boson $\left(\sigma_{H / A}\right)$ times its branching ratio into SM states. In this analysis, we consider the latest search limits on $\sigma_{g g H} \times B r(H \rightarrow Z Z, W W, \tau \tau)[132,195-197], \sigma_{b b H / A} \times B r(H / A \rightarrow$ $\tau \tau)$ [132, 197], $p p \rightarrow H \rightarrow \gamma \gamma$ [198] and $p p \rightarrow H \rightarrow h h \rightarrow 4 b, 2 b 2 \gamma, 2 b 2 \tau$ [123, 134, 136] derived by the CMS and ATLAS collaborations upon using the Run-II dataset with an integrated luminosity of $\sim 36 \mathrm{fb}^{-1}$. The gluon fusion channel is undoubtedly the dominant Higgs production mode at the LHC for low values of $\tan \beta$. However, it gets overrun by the $b \bar{b} H / A$ production channel at high $\tan \beta$ values. In the current analysis, while evaluating the impact of the existing upper limits on $p p \rightarrow H \rightarrow h h \rightarrow 4 b, 2 b 2 \gamma, 2 b 2 \tau$, only the contributions from the gluon fusion production are taken into account. This choice is motivated by the fact that the $H \rightarrow h h$ decay modes gain dominance only in the low and intermediate $\tan \beta$ values where the gluon fusion mode overshadows the $b \bar{b} H / A$ channel. Although the current search limits on $H \rightarrow h h$ do not impose any constraints on our parameter space, the future runs have the potential to probe the low $m_{A}$ and low $\tan \beta$ regime. The impact of these future limits are discussed in the later part of this section. The $H \rightarrow Z Z / W W$ limits also turn out to be ineffective in constraining our parameter space and will require improvements of about three orders of magnitude for making any impact. We would like to mention that the upper limits derived by ATLAS in the $H \rightarrow \gamma \gamma$ search channel is on the fiducial cross-section times $\operatorname{BR}(H \rightarrow \gamma \gamma)$. We compare these upper limits against a combination of the $g g F+b \bar{b} H / A$ production cross-sections and observe that an improvement of around two orders of magnitude will be required in order to affect our parameter space. Limits from searches in the $H / A \rightarrow \tau \tau$ channel impose the strongest constraints on the parameter space. Constraints from $\sigma_{b \bar{b} H / A} \times \mathrm{BR}(H / A \rightarrow \tau \tau)$ yield stronger limits compared to their gluon fusion counterparts and exclude the low $m_{A}$ and high $\tan \beta$ region. The current search limits from ATLAS and CMS furnish roughly equivalent impact and rule out $\tan \beta \gtrsim 16$ for $m_{A} \sim 1 \mathrm{TeV}$. Before presenting the results in the $m_{A}-\tan \beta$ plane, we show the current allowed branching fractions, viz., $H \rightarrow h h, H \rightarrow t \bar{t}$ and $H \rightarrow \tau^{+} \tau^{-}$in figure 39. The $H \rightarrow h h$ branching ratio dominates for $\tan \beta \lesssim 8$ and for $m_{H} \leq 2 m_{t}$. All points are allowed by the Higgs mass and Higgs signal strength constraints. However, the grey regions are excluded by the present direct searches of the heavy Higgs. In figure 38, we show the impact of the latest direct search limits from $\sigma_{b \bar{b} H / A} \times \operatorname{Br}(H / A \rightarrow \tau \tau)$ in the $m_{A}-\tan \beta$ plane. The parameter space points shown in figure 38 (grey and orange) are obtained after implementing the light Higgs mass constraints and the Higgs signal strength measurements. The grey points are excluded upon imposing the aforementioned direct search limits.

Our main concern in this section is to quantify the impact of the projected direct search limits for the HL-LHC which were derived in the previous sections. In this regard, we consider the projected direct search limits for the HL-LHC in the $H \rightarrow h h$ (section 2), $H \rightarrow t \bar{t}$ (section 3) and $b \bar{b} H / A \rightarrow b \bar{b} \tau_{h} \tau_{h}$ (section 4) channels. Among the various final states of the $H \rightarrow h h$ channel, the $b \bar{b} \gamma \gamma$ final state furnishes the strongest limit in the $m_{A} \lesssim 600 \mathrm{GeV}$ regime, while the $4 b$ final state imposes the strongest upper limits in the $m_{A} \gtrsim 600 \mathrm{GeV}$ region. The $H \rightarrow h h$ decay mode gains dominance in the low $\tan \beta$ region and especially before the $t \bar{t}$ mass threshold is attained. The same is reflected in the left 

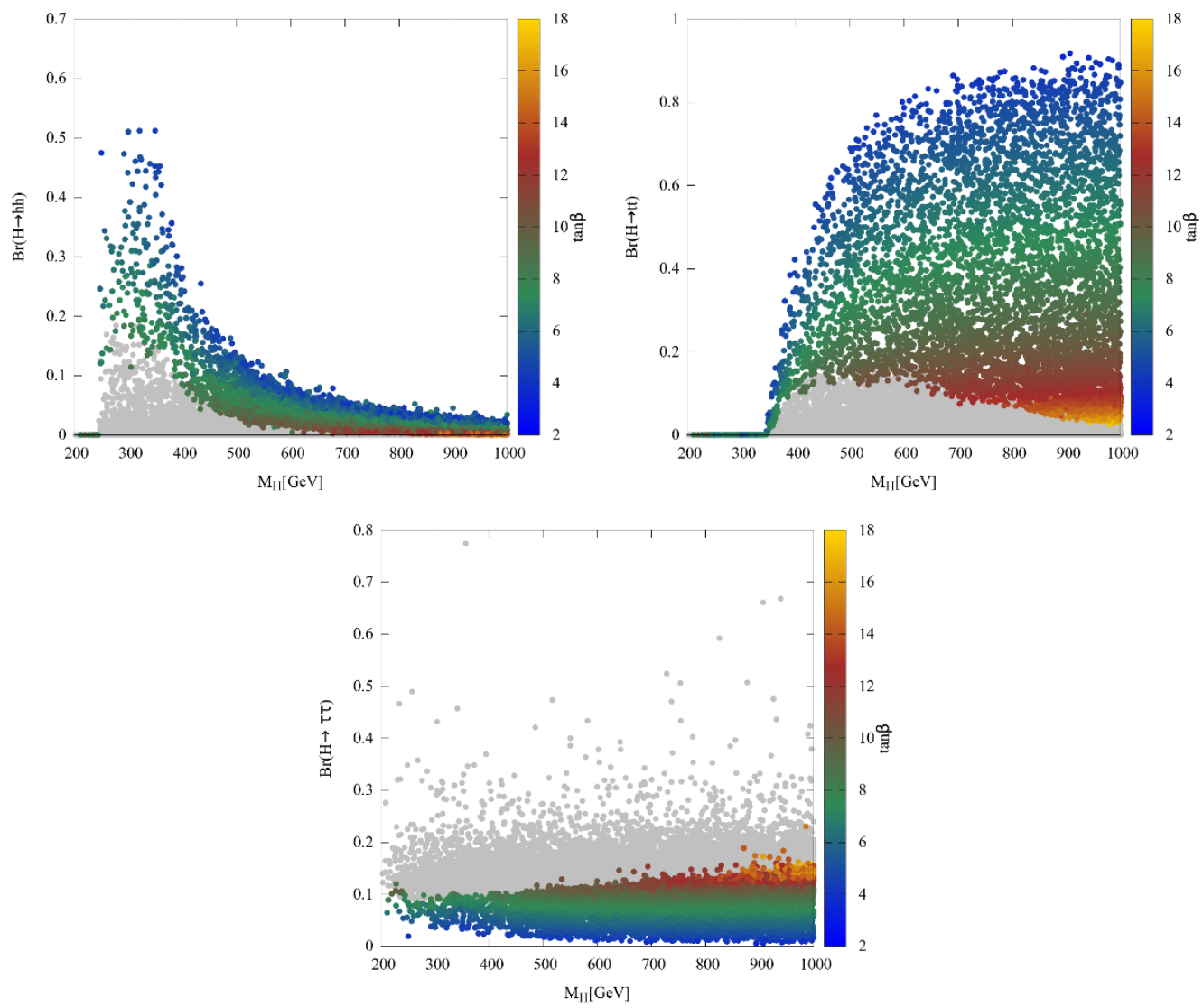

Figure 39. Branching ratios of $H \rightarrow h h, H \rightarrow t \bar{t}$ and $H \rightarrow \tau^{+} \tau^{-}$as a function of $m_{H}$. All the points are allowed by the SM-like Higgs mass and Higgs signal strength constraints. The grey points are excluded by the present direct searches for the heavy Higgs boson.

panel of figure 40 where the brown points represent the region excluded at 95\% CL by the projected $2 \sigma$ reach from the $H \rightarrow h h \rightarrow b \bar{b} \gamma \gamma$ channel. The $4 b$ final state, on the other hand, is rendered ineffective on account of reduced production cross-section at high values of $m_{A}$. The upper limits derived from searches in the remaining $H \rightarrow h h$ channels furnish much weaker bounds and will not be able to probe the pMSSM parameter even at the HL-LHC. The couplings of the heavy Higgs bosons with the up-type quarks have an inverse dependence on $\tan \beta$ and thus consequently the $H \rightarrow t \bar{t}$ channel has the potential to play an important role in the low $\tan \beta$ regime. The parameter space points excluded at $95 \%$ CL by the $H \rightarrow t \bar{t}$ HL-LHC search limits derived in section 3 are shown in green in figure 40. The strongest future limits are obtained by the $b \bar{b} H \rightarrow b \bar{b} \tau \tau$ channel (derived in section 4). This will be able to exclude (at $2 \sigma$ ) until $\sim \tan \beta \sim 5.5$ at $m_{A} \sim 1 \mathrm{TeV}$ as shown in figure 40 , where the orange points are excluded by the same. The blue points in figure 40 denotes the parameter space which will evade the direct searches at the HL-LHC as well. The right panel in figure 40, however, shows the discovery potential at $5 \sigma$.

At this point, we would like to briefly discuss the implications from direct charged Higgs search limits on the parameter space of our interest. A detailed analysis of the exclusion 

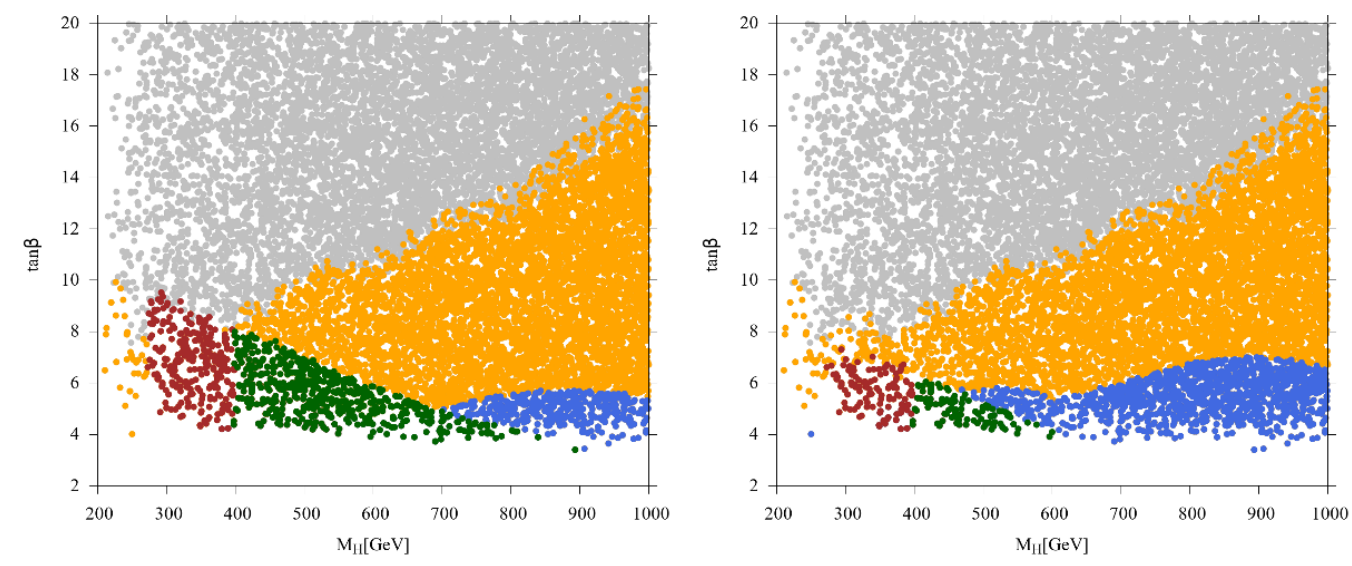

Figure 40. Scatter plot in the $m_{A}-\tan \beta$ plane showing the impact of the projected search limits derived in this study, for the case of HL-LHC. The orange and grey colored points represent the same color code of figure 38. The brown colored points are excluded by the $2 \sigma$ upper limits on $\sigma_{g g H} \times \operatorname{Br}(H \rightarrow h h) \times \operatorname{Br}(h \rightarrow b \bar{b}) \times B r(h \rightarrow \gamma \gamma)$, derived in section 2.1, while the green colored points are excluded by the $2 \sigma$ upper limits on $\sigma_{g g H} \times B r(H \rightarrow t \bar{t})$ derived in section 3. Upper limits derived for the case of $\sigma_{b \bar{b} H / A} \times B r(H / A \rightarrow \tau \tau)$ (section 4) at $2 \sigma$ rule out the orange colored points. The blue colored points represent the parameter space which would remain allowed after the HL-LHC run. The left and the right plots respectively show the exclusion at $2 \sigma$ and discovery reach at $5 \sigma$.

reach of current limits from direct charged Higgs searches in the $p p \rightarrow H^{ \pm} \rightarrow \tau^{ \pm} \nu_{\tau}$ and $p p \rightarrow H^{+} \rightarrow t \bar{b}$ can be found in [39, 169]. Figure 11 and figure 12 of [39] shows that the allowed MSSM parameter space points (obtained by imposing the light Higgs mass constraint, Higgs signal strength limits and flavour physics constraints) are outside the current reach of charged Higgs searches in the $\tau \nu_{\tau}$ and $t \bar{b}$ channels, respectively, and, the direct charged Higgs search limits would require an improvement of roughly an order of magnitude in order to be capable of probing some of the MSSM parameter space points considered in [39]. The implications for future direct charged Higgs search limits for HLLHC, obtained by scaling the current limits, has been analysed in [169], where the projected reach of direct searches in the $H^{ \pm} \rightarrow \tau^{ \pm} \nu_{\tau}$ and $H^{ \pm} \rightarrow t \bar{b}$ channels has been translated to the MSSM parameter space and presented in the $M_{A}-\tan \beta$ plane (see figure 20 of [169]). A comparison with the analysis in [169] indicates that the future reach of direct charged Higgs searches at the HL-LHC is weaker than the future reach of direct heavy Higgs searches in the $b \bar{b} H / A, H / A \rightarrow \tau^{+} \tau^{-}$channel derived in this work.

The results discussed till now assume that the heavy Higgs bosons underwent decays only into SM final states. The branching fractions of the heavy Higgs bosons into SM final states can, in principle, undergo significant modifications in the presence of light SUSY particles. ${ }^{12}$ For example, for intermediate values of $\tan \beta \sim 7-10$, the branching fraction of the heavy Higgs bosons into charginos and neutralinos may attain significantly large values ( $\gtrsim 50 \%$ ) [209, 210]. In the remainder of this section we study the impact on the pMSSM

\footnotetext{
${ }^{12}$ SUSY particles with their masses less than $M_{H / A} / 2$, such that it is kinematically possible for the heavy Higgs bosons to decay into them, are referred to as the light SUSY particles.
} 


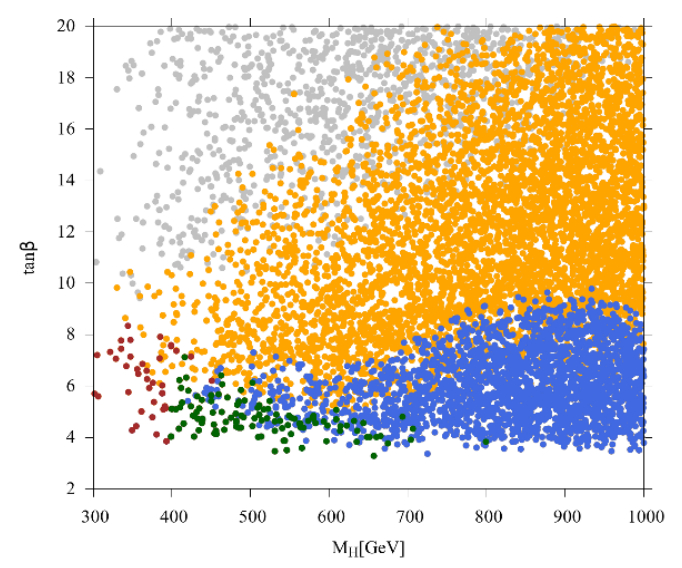

Figure 41. Same as the left panel of figure 40 but for $\left|M_{2}-\mu\right|<10 \mathrm{GeV}, M_{H / A}>\left(M_{\chi_{1}^{0}}+M_{\chi_{2}^{0}}\right)$ and $M_{2}, \mu>200 \mathrm{GeV}$.

parameter space in the presence of non-SM decay modes of the heavy Higgs bosons at the HL-LHC. Here we will restrict ourselves to the case of light electroweakinos. These electroweakinos are required to be an admixture of gauginos (bino and wino) and higgsinos in order to have couplings with the Higgs bosons. The pure gauginos and higgsinos do not couple with the Higgs states. LHC searches in the chargino-neutralino pair production mode furnishes the most stringent constraints on the electroweakino sector and excludes degenerate wino-like $\chi_{2}^{0}$ and $\chi_{1}^{ \pm}$of mass $\lesssim 450 \mathrm{GeV}$ [194], for an LSP neutralino of mass $\sim 100 \mathrm{GeV}$. However, such constraints do not apply to scenarios where the LSP and NLSP are almost degenerate in mass. We explore this fact and vary $M_{2}$ and $\mu$ in such a way that $\left|M_{2}-\mu\right|<10 \mathrm{GeV}, M_{H / A}>\left(\chi_{1}^{0}+\chi_{2}^{0}\right)$ and $M_{2}, \mu>200 \mathrm{GeV}$ [194]. Closeness between $M_{2}$ and $\mu$ ensures that the $\chi_{1}^{0}, \chi_{2}^{0}, \chi_{3}^{0}$ and $\chi_{1}^{ \pm}$have significant admixtures from both winos and higgsinos. The remaining input parameters are randomly varied within the range specified in eq. (5.2) except for $M_{1}$ which we fix at $1 \mathrm{TeV}$. In presence of these $H / A \rightarrow$ ino decay modes, the branching fraction of the heavy Higgs bosons to SM final states undergoes modifications and manifests in weaker limits on the parameter space, as shown in figure 41. Correspondingly, the orange and blue regions shift upward. The brown and green regions shrink further down. For $m_{A}$ varying between $400 \mathrm{GeV}$ and $700 \mathrm{GeV}, \tan \beta$ as low as 3 is excluded at $95 \% \mathrm{CL}$. In presence of these non-SM decay modes, the current $(13 \mathrm{TeV}$, $\left.36 \mathrm{fb}^{-1}\right)$ limits on $\sigma_{b \bar{b} H / A} \times B r(H / A \rightarrow \tau \tau)$ exclude $\tan \beta \gtrsim 22$ for $m_{A} \sim 1 \mathrm{TeV}$. The HL-LHC reach weakens out till $\tan \beta \sim 10$ at $m_{A} \sim 1 \mathrm{TeV}$. In the current scenario, the projected limits from $H \rightarrow t \bar{t}$ lose sensitivity on the pMSSM parameter space under study. The HL-LHC projections from $H \rightarrow h h \rightarrow b \bar{b} \gamma \gamma$ also imposes weaker constraints and excludes $\tan \beta<8$ at $m_{A} \sim 400 \mathrm{GeV}$.

\section{Summary}

In this work, we have studied the prospects of observing or excluding a resonant heavy Higgs or pseudoscalar in the purview of the HL-LHC at $14 \mathrm{TeV}$. Various experimental observations and theoretical motivations necessitate physics beyond the Standard Model 
(BSM). Several searches are performed, either in the context of specific models or in a generic model-independent fashion, to gauge the type of new physics. In this work, we specifically focus on neutral heavy Higgs bosons (both $C P$ odd and even). Run-II data at the LHC has already constrained strongly interacting BSM particles like gluinos and stops to $\mathcal{O}(\geq 1) \mathrm{TeV}$. However, the LHC still hasn't imposed such strong constraints on extended Higgs sectors. The Standard Model Higgs self-coupling being still unknown, we are yet to fully understand the scalar sector of new physics. Here, we studied three major search channels for such a heavy Higgs (or pseudoscalar). Specific to the $C P$-even heavy Higgs, we studied the prospects of constraining $\sigma(p p \rightarrow H \rightarrow h h)$ in multifarious channels, viz., $b \bar{b} \gamma \gamma$, $b \bar{b} b \bar{b}, b \bar{b} \tau^{+} \tau^{-}, b \bar{b} W W^{*}$ and $\gamma \gamma W W^{*}$. We took guidance from the present searches and optimised each channel carefully to obtain upper limits on $\sigma(p p \rightarrow H \rightarrow h h)$ from each channel. Corroborating the present searches, we find that the $b \bar{b} \gamma \gamma$ and $b \bar{b} b \bar{b}$ final states serve as the golden channels for $m_{H}$ in the range $[300,600] \mathrm{GeV}$ and $[600,1000] \mathrm{GeV}$, respectively. The $b \bar{b} \gamma \gamma$ sets a $95 \%$ CL upper limit on $\sigma(p p \rightarrow H \rightarrow h h)$ between $[79.03,14.10]$ fb in the aforementioned mass range. The $4 b$ channel on the other hand sets a corresponding cross-section limit between $[5.36,2.51] \mathrm{fb}$ for $m_{H} \in[800,1000] \mathrm{GeV}$. The limits from the remaining three channels are not so promising. On the other hand, if the mass of the scalar or the pseudoscalar Higgs is above the $t \bar{t}$ threshold and the Higgs is dominantly produced via gluon fusion, then it can also have a dominant decay into $t \bar{t}$. Upon studying the fully leptonic as well as the semi-leptonic final states, we find the strongest limits on $\sigma(p p \rightarrow H \rightarrow t \bar{t})$ from the semi-leptonic category. The $95 \% \mathrm{CL}$ upper limits lie between $186.57 \mathrm{fb}$ and $32.81 \mathrm{fb}$ for $m_{H} \in[400,1000] \mathrm{GeV}$. Finally, we studied the $b \bar{b} H / A$ production in the $b \bar{b} \tau \tau$ final state upon demanding at least one $b$-tagged jet in the final state and demanding two hadronic $\tau \mathrm{s}$. The 95\% CL upper limit on $\sigma(p p \rightarrow b \bar{b} H)$ varies between $22.16 \mathrm{fb}$ and $3.68 \mathrm{fb}$ for $m_{H}$ lying between $300 \mathrm{GeV}$ and $500 \mathrm{GeV}$. All our searches for the scalars are mostly model independent and can be translated to models with multiple Higgs bosons with narrow widths.

In this work, we considered the specific example of supersymmetry, more specifically the pMSSM. We apply present constraints from the SM-like Higgs boson mass measurement and all its signal strengths into multiple final states. The future limits obtained in this work constrain different regimes of the parameter space. The $H \rightarrow h h$ search, mostly in the $b \bar{b} \gamma \gamma$ channel excludes $\tan \beta$ to as low as 4 , at $95 \% \mathrm{CL}$, for $m_{A} \sim 2 m_{t} \mathrm{GeV}$. The $H \rightarrow t \bar{t}$ has a similar exclusion on $\tan \beta$ for $m_{A}$ varying between $[400,800] \mathrm{GeV}$. The $b \bar{b} H$ channel in the di- $\tau+\geq 1 b$-tagged jet final state excludes $\tan \beta$ as low as 5.5 for $m_{A}=1 \mathrm{TeV}$. The blue region in figure 40 will not be probed even by direct searches if the heavy Higgs bosons decay only to SM particles. We will require higher energy colliders in order to be able to probe this region. This scenario might change if there are light electroweakinos, below $m_{H / A} / 2$. In that situation, the $m_{A}-\tan \beta$ parameter region changes. Upon considering a scenario where $\left|M_{2}-\mu\right|<10 \mathrm{GeV}, M_{H / A}>\left(\chi_{1}^{0}+\chi_{2}^{0}\right)$ and $M_{2} / \mu>200 \mathrm{GeV}$, one finds that the $H \rightarrow h h$ and $H \rightarrow t \bar{t}$ excludes $\tan \beta$ down to 3 for $m_{A} \in[400,700] \mathrm{GeV}$. The exclusion bound on $\tan \beta$ from the $b \bar{b} H$ search decreases to 10 for $m_{A}=1 \mathrm{TeV}$ at $95 \% \mathrm{CL}$. 


\section{A Detailing the cross section with generation cuts for the signal and backgrounds}

\begin{tabular}{|c|c|c|c|}
\hline Process & Backgrounds & $\begin{array}{l}\text { Generation-level cuts }\left(\ell=e^{ \pm}, \mu^{ \pm}\right) \\
\text {(NA : Not Applied) }\end{array}$ & Cross section $(\mathrm{fb})$ \\
\hline \multicolumn{4}{|c|}{$p p \rightarrow H \rightarrow h h, p p \rightarrow A \rightarrow Z h$ and $p p \rightarrow H \rightarrow t \bar{t}$ final states } \\
\hline \multirow{13}{*}{$b \bar{b} \gamma \gamma$} & $h h \rightarrow b \bar{b} \gamma \gamma$ & NA & 0.10 \\
\hline & $b \bar{b} \gamma \gamma+$ jets & $\begin{array}{c}p_{T, j / b / \gamma}>20 \mathrm{GeV},\left|\eta_{j}\right|<5.0,\left|\eta_{b, \gamma}\right|<2.5 \\
\Delta R_{b, j, \gamma}{ }^{a}>0.2, m_{b b}>50 \mathrm{GeV}, 110<m_{\gamma \gamma}<140 \mathrm{GeV}\end{array}$ & 18.78 \\
\hline & $c \bar{c} \gamma \gamma$ & $\begin{array}{c}p_{T, j / b / \gamma}>20 \mathrm{GeV},\left|\eta_{j}\right|<5.0,\left|\eta_{\gamma}\right|<2.5 \\
\Delta R_{b, j, \gamma}>0.2,110<m_{\gamma \gamma}<140 \mathrm{GeV}\end{array}$ & 162.22 \\
\hline & $j j \gamma \gamma$ & same as $c \bar{c} \gamma \gamma$ & $2770.67^{*}$ \\
\hline & $t \bar{t} h, h \rightarrow \gamma \gamma$ & NA & 1.39 \\
\hline & $h b \bar{b}, h \rightarrow \gamma \gamma$ & NA & 1.32 \\
\hline & $Z h, h \rightarrow \gamma \gamma, Z \rightarrow b \bar{b}$ & NA & 0.33 \\
\hline & $b \bar{b} j j$ & $\begin{aligned} p_{T, j}> & 10 \mathrm{GeV}, p_{T, b}>20 \mathrm{GeV},\left|\eta_{j / b}\right|<5.0 \\
& m_{j j}>50 \mathrm{GeV}, m_{b b}>50 \mathrm{GeV}\end{aligned}$ & $549583730.00^{*}$ \\
\hline & $b \bar{b} j \gamma$ & $\begin{array}{c}p_{T, j / b / \gamma}>20 \mathrm{GeV},\left|\eta_{j}\right|<5.0,\left|\eta_{b / \gamma}\right|<2.5 \\
\Delta R_{b / b / \gamma / \gamma, b / j / j / b}>0.2, m_{b b}>50 \mathrm{GeV}\end{array}$ & $201800^{*}$ \\
\hline & $c \bar{c} j \gamma$ & $p_{T, j / \gamma}>20 \mathrm{GeV},\left|\eta_{j}\right|<5.0,\left|\eta_{\gamma}\right|<2.5, \Delta R_{j, \gamma}>0.2$ & $1132709.63^{*}$ \\
\hline & $Z \gamma \gamma+$ jet, $Z \rightarrow b \bar{b}$ & $\begin{array}{c}p_{T, j / b / \gamma}>20 \mathrm{GeV},\left|\eta_{j}\right|<5.0,\left|\eta_{b / \gamma}\right|<2.5 \\
\Delta R_{b, j, \gamma}>0.2, m_{b b}>50 \mathrm{GeV}, 110<m_{\gamma \gamma}<140 \mathrm{GeV}\end{array}$ & 0.87 \\
\hline & $g g \rightarrow h+c \bar{c}, h \rightarrow \gamma \gamma$ & $p_{T, j}>20 \mathrm{GeV},\left|\eta_{j}\right|<5.0, \Delta R_{j, j}>0.2$ & 0.31 \\
\hline & $g g \rightarrow h+j j, h \rightarrow \gamma \gamma$ & same as $g g \rightarrow h+c \bar{c}$ & $27.89^{*}$ \\
\hline \multirow{6}{*}{$b \bar{b} b \bar{b}$} & $h h \rightarrow b \bar{b} b \bar{b}$ & NA & 13.42 \\
\hline & multijet $b \bar{b} b \bar{b}$ & $\begin{array}{c}p_{T, j / b}>50 \mathrm{GeV},\left|\eta_{j / b}\right|<3.0, \Delta R_{b, j}>0.3 \\
H_{T}>250 \mathrm{GeV}\end{array}$ & 14541.30 \\
\hline & multijet $b \bar{b} c \bar{c}$ & same as multijet $b \bar{b} b \bar{b}$ & 28633.60 \\
\hline & multijet $b \bar{b} j j$ & same as multijet $b \bar{b} b \bar{b}$ & $3602560.00^{*}$ \\
\hline & $t \bar{t}, W^{ \pm} \rightarrow c(\bar{c}) \bar{s}(s)$ & same as multijet $b \bar{b} b \bar{b}$ & 860.17 \\
\hline & $t \bar{t} b \bar{b}$ & $p_{T, b}>50 \mathrm{GeV},\left|\eta_{b}\right|<3.0, \Delta R_{b, b}>0.3, H_{T}>250 \mathrm{GeV}$ & 170.58 \\
\hline \multirow{10}{*}{$b \bar{b} \tau^{+} \tau^{-}$} & $h h \rightarrow b \bar{b} \tau^{+} \tau^{-}$ & NA & 2.89 \\
\hline & $t \bar{t}$ hadronic & $\begin{array}{l}p_{T, j / b}>20 \mathrm{GeV}, p_{T, l}>8 \mathrm{GeV},\left|\eta_{j}\right|<5.0 \\
\left|\eta_{b / \ell}\right|<3.0, \Delta R_{b, j, \ell}>0.2, m_{b b}>50 \mathrm{GeV}\end{array}$ & 135623.50 \\
\hline & $t \bar{t}$ semi-leptonic & same as $t \bar{t}$ hadronic & 173409.88 \\
\hline & $\tau \tau b \bar{b}$ & $\begin{array}{c}p_{T, b}>20 \mathrm{GeV}, p_{T, \tau}>8 \mathrm{GeV},\left|\eta_{b / \tau}\right|<3.0 \\
\Delta R_{b, \tau}>0.2, m_{b b}>50 \mathrm{GeV}, m_{\tau \tau}>30 \mathrm{GeV}\end{array}$ & 2128.56 \\
\hline & $b \bar{b} h, h \rightarrow \tau \tau$ & $\begin{array}{c}p_{T, b}>20 \mathrm{GeV}, p_{T, \tau}>10 \mathrm{GeV},\left|\eta_{j}\right|<5.0 \\
\left|\eta_{b / \tau}\right|<3.0, \Delta R_{b, \tau}>0.2, m_{b b}>50 \mathrm{GeV}\end{array}$ & 1.23 \\
\hline & $\mathrm{Zh}, h \rightarrow(b \bar{b}+\tau \tau), Z \rightarrow(\tau \tau+b \bar{b})$ & NA & 28.21 \\
\hline & $t \bar{t} h$ & NA & 611.30 \\
\hline & $t \bar{t} Z$ & NA & 731.54 \\
\hline & $t \bar{t} W$ & NA & 437.87 \\
\hline & $b \bar{b} j j$ & $\begin{array}{c}p_{T, j}>10 \mathrm{GeV}, p_{T, b}>20 \mathrm{GeV},\left|\eta_{j / b}\right|<5.0 \\
m_{j j}>50 \mathrm{GeV}, m_{b b}>50 \mathrm{GeV}\end{array}$ & $549583730.00^{*}$ \\
\hline
\end{tabular}

${ }^{a} \Delta R_{b, j, \gamma}$ means $\Delta R$ between all possible combination of $b, j$ and $\gamma$.

${ }^{b} \Delta R_{a / b, c / d}$ signifies $\Delta R_{a c}$ and $\Delta R_{b d}$.

Table 19. Generation level cuts and cross-sections for the various backgrounds used in the analyses. The backgrounds labelled with $*$ are multiplied by the fake rates before doing the analysis. The fake rates used are $0.05 \%$ [61] for $j \rightarrow \gamma, \sim 1.75 \%$ (average from the fake rate function) for $j \rightarrow b$ and $0.35 \%[211]$ for $j \rightarrow \tau$. 


\begin{tabular}{|c|c|c|c|}
\hline Process & Backgrounds & $\begin{array}{l}\text { Generation-level cuts }\left(\ell=e^{ \pm}, \mu^{ \pm}\right) \\
\text {(NA : Not Applied) }\end{array}$ & Cross section $(\mathrm{fb})$ \\
\hline \multicolumn{4}{|c|}{$p p \rightarrow H \rightarrow h h, p p \rightarrow A \rightarrow Z h$ and $p p \rightarrow H \rightarrow t \bar{t}$ final states } \\
\hline \multirow{8}{*}{$b \bar{b} W W^{*}$} & $h h \rightarrow b \bar{b} W^{+} W^{-}$ & NA & 9.85 \\
\hline & $t \bar{t}$ semi-leptonic & $\begin{array}{l}p_{T, j / b}>20 \mathrm{GeV}, p_{T, l}>8 \mathrm{GeV},\left|\eta_{j}\right|<5.0 \\
\left|\eta_{b / \ell}\right|<3.0, \Delta R_{b, j, \ell}>0.2, m_{b b}>50 \mathrm{GeV}\end{array}$ & 173409.88 \\
\hline & $t \bar{t}$ leptonic & same as $t \bar{t}$ semileptonic & 55319.44 \\
\hline & $\ell \ell b \bar{b}$ & $\begin{array}{c}p_{T, b}>20 \mathrm{GeV}, p_{T, l}>8 \mathrm{GeV},\left|\eta_{b / \ell}\right|<3.0 \\
\Delta R_{b, \ell}>0.2, m_{b b}>50 \mathrm{GeV}\end{array}$ & 7393.72 \\
\hline & $W b b+j e t s, W \rightarrow \ell \nu, \ell$ also includes $\tau$ & $\begin{array}{c}p_{T, j / b}>20 \mathrm{GeV}, p_{T, l}>8 \mathrm{GeV},\left|\eta_{j}\right|<5.0 \\
\left|\eta_{b / \ell}\right|<3.0, \Delta R_{j, b, l}>0.2\end{array}$ & 32576.60 \\
\hline & $t \bar{t} h$ & NA & 611.30 \\
\hline & $t \bar{t} Z$ & NA & 731.54 \\
\hline & $t \bar{t} W$ & NA & 437.87 \\
\hline \multirow{6}{*}{$\gamma \gamma W W^{*}$} & $h h \rightarrow \gamma \gamma W^{+} W^{-}$ & NA & 0.04 \\
\hline & $t \bar{t} h, h \rightarrow \gamma \gamma$ & NA & 1.39 \\
\hline & $Z h+$ jets, $h \rightarrow \gamma \gamma, Z \rightarrow \ell \ell(\ell$ includes $\tau$ also $)$ & $\begin{array}{c}p_{T, \gamma / \ell}>10 \mathrm{GeV},\left|\eta_{j}\right|<5.0,\left|\eta_{\gamma / \ell}\right|<2.5 \\
\Delta R_{\gamma, \ell, j}>0.2,120 \mathrm{GeV}<m_{\gamma \gamma}<130 \mathrm{GeV}\end{array}$ & 0.12 \\
\hline & $W h+$ jets, $h \rightarrow \gamma \gamma, W \rightarrow \ell \nu(\ell$ includes $\tau$ also $)$ & same as $Z h+$ jets & 0.70 \\
\hline & $\ell \nu \gamma \gamma+$ jets, $\ell$ also includes $\tau$ & $\begin{array}{l}p_{T, \gamma / \ell}>10 \mathrm{GeV},\left|\eta_{j}\right|<5.0,\left|\eta_{\gamma / \ell}\right|<2.5, \Delta R_{\gamma \gamma}>0.2, \\
\Delta R_{\gamma \ell}>0.2, \Delta R_{\gamma j}>0.4,120 \mathrm{GeV}<m_{\gamma \gamma}<130 \mathrm{GeV}\end{array}$ & 3.17 \\
\hline & $\ell \ell \gamma \gamma+$ jets, $\ell$ also includes $\tau$ & same as $\ell \nu \gamma \gamma+$ jets, with $m_{\ell \ell}>20 \mathrm{GeV}$ & 1.00 \\
\hline \multicolumn{4}{|c|}{$p p \rightarrow b \bar{b} H$ final state } \\
\hline \multirow{8}{*}{$b \bar{b} \tau^{+} \tau^{-}$} & $t \bar{t}$ & $p_{T, b}>20 \mathrm{GeV},\left|\eta_{b}\right|<3.0, \Delta R_{b b}>0.2$ & 633946.81 \\
\hline & single top s-channel & NA & 11390.00 \\
\hline & single top t-channel & NA & 248090.00 \\
\hline & single top Wt-channel & NA & 84400.00 \\
\hline & $\tau \tau+$ jets, via $Z / \gamma^{*}$ & $\begin{array}{c}p_{T, j / b}>20 \mathrm{GeV}, p_{T, \ell}>60 \mathrm{GeV},\left|\eta_{j / b / \ell}\right|<3.0 \\
\Delta R_{j, b, \ell}>0.2, m_{\ell \ell}>50 \mathrm{GeV}\end{array}$ & 884370.24 \\
\hline & $W+$ jets, $W \rightarrow \ell \nu(\ell$ includes $\tau$ also $)$ & $p_{T, j / b / \ell}>20 \mathrm{GeV},\left|\eta_{j / b / \ell}\right|<3.0, \Delta R_{j, b, \ell}>0.2$ & 112358.64 \\
\hline & $V V\left(V\right.$ includes $W^{ \pm}$and $\left.Z\right)$ & NA & 106510.72 \\
\hline & $b \bar{b} j j$ & $\begin{array}{c}p_{T, j}>65 \mathrm{GeV}, p_{T, b}>20 \mathrm{GeV},\left|\eta_{j / b}\right|<3.0 \\
\Delta R_{j, b}>0.2, m_{j j}>50 \mathrm{GeV}\end{array}$ & $12091572.60^{*}$ \\
\hline
\end{tabular}

Table 20. Generation level cuts and cross-sections for the signals and various backgrounds used in the analyses. The backgrounds labelled with $*$ are multiplied by the fake rates before doing the analysis. The fake rates used are $0.35 \%$ [211] for $j \rightarrow \tau$.

\section{B Validation of the $b \bar{b} H$ analysis}

Before performing our analysis for the $b \bar{b} H$ category, we validate our setup with the existing analysis in this channel [132, 212, 213]. We generate the signal events (at LO in SM) with MG5_aMC@NLO and shower them via Pythia-8 [214]. We use different parton distribution functions (PDFs) for the sample generations. Specifically, we use the CT10nlo_nf4 [215] for the $4 \mathrm{~F} b \bar{b} H$ process, MSTW2008nnlo68cl [216] for the $5 \mathrm{~F} b \bar{b} H$ process and CT10 [217] for the ggF process. Next, we impose the following cuts in sequence. For the $b$-tag category, we demand at least one $b$-tagged jet in the final state. The events are required to have at least two $\tau$ jets with opposite charge (from their reconstructed charged tracks). The leading and sub-leading $\left(p_{T}\right.$ ordered) $\tau$-tagged jets are required to have $p_{T}>65 \mathrm{GeV}$. The $\tau$ jets lying inside the transition region viz., $1.37<|\eta|<1.52$, are removed. Furthermore, the 


\begin{tabular}{|c|c|c|}
\hline Process & Event rates at $13 \mathrm{TeV}$ with $36.1 \mathrm{fb}^{-1}$ of integrated luminosity & Total \\
\hline & b-tag category & \\
\hline multijet & 97.06 & \multirow{5}{*}{215.26} \\
\hline$Z / \gamma^{*}+$ jets & 11.03 & \\
\hline$W+$ jets & 2.82 & \\
\hline$t \bar{t}$ & 83.66 & \\
\hline$V V+$ jets & 1.87 & \\
\hline $500 \mathrm{GeV}$ Signal & 18.82 & \\
\hline \multicolumn{3}{|c|}{ ATLAS numbers [132] } \\
\hline multijet & $106 \pm 32$ & \multirow{5}{*}{$180 \pm 60$} \\
\hline$Z / \gamma^{*} \rightarrow \tau \tau$ & $7.5 \pm 2.9$ & \\
\hline$W(\rightarrow \tau \nu)+$ jets & $4.0 \pm 1.0$ & \\
\hline$t \bar{t}+$ single top & $60 \pm 50$ & \\
\hline Others & $1.0 \pm 0.5$ & \\
\hline $500 \mathrm{GeV}$ Signal & $28 \pm 12$ & \\
\hline
\end{tabular}

Table 21. Comparison table for the $\tau_{h} \tau_{h}$ channel in the $b$-tag category.

azimuthal angle separation between the leading and the sub-leading $\tau$ jets is required to be $\Delta \phi(\tau, \tau)>2.7$. Furthermore, we require $m_{\tau \tau}^{\text {vis. }}>50 \mathrm{GeV}, \mathbb{E}_{T}>20 \mathrm{GeV}$ and $\sum_{\tau} \cos (\Delta \phi)>0$. We show the validation in table 21 .

\section{Acknowledgments}

We thank Mikael Chala, Arghya Choudhury and Marius Wiesemann for helpful discussions at various stages of the work. The work of BB is supported by the Department of Science and Technology, Government of India, under the Grant Agreement number IFA13-PH-75 (INSPIRE Faculty Award). SB is supported by a Durham Junior Research Fellowship COFUNDed by Durham University and the European Union, under grant agreement number 609412 . 
Open Access. This article is distributed under the terms of the Creative Commons Attribution License (CC-BY 4.0), which permits any use, distribution and reproduction in any medium, provided the original author(s) and source are credited.

\section{References}

[1] ATLAS and CMS collaborations, Measurements of the Higgs boson production and decay rates and constraints on its couplings from a combined ATLAS and CMS analysis of the LHC pp collision data at $\sqrt{s}=7$ and 8 TeV, JHEP 08 (2016) 045 [arXiv:1606.02266] [INSPIRE].

[2] CMS collaboration, Updated measurements of Higgs boson production in the diphoton decay channel at $\sqrt{s}=13 \mathrm{TeV}$ in pp collisions at CMS., CMS-PAS-HIG-16-020.

[3] ATLAS collaboration, Measurement of fiducial, differential and production cross sections in the $H \rightarrow \gamma \gamma$ decay channel with $13.3 \mathrm{fb}^{-1}$ of $13 \mathrm{TeV}$ proton-proton collision data with the ATLAS detector, ATLAS-CONF-2016-067.

[4] ATLAS collaboration, Study of the Higgs boson properties and search for high-mass scalar resonances in the $H \rightarrow Z Z^{*} \rightarrow 4 \ell$ decay channel at $\sqrt{s}=13$ TeV with the ATLAS detector, ATLAS-CONF-2016-079.

[5] ATLAS collaboration, Combined measurements of the Higgs boson production and decay rates in $H \rightarrow Z Z^{*} \rightarrow 4 \ell$ and $H \rightarrow \gamma \gamma$ final states using pp collision data at $\sqrt{s}=13 \mathrm{TeV}$ in the ATLAS experiment, ATLAS-CONF-2016-081.

[6] CMS collaboration, Measurements of properties of the Higgs boson decaying into the four-lepton final state in pp collisions at $\sqrt{s}=13$ TeV, JHEP 11 (2017) 047 [arXiv: 1706.09936] [INSPIRE].

[7] ATLAS collaboration, Measurements of the Higgs boson production cross section via Vector Boson Fusion and associated $W H$ production in the $W W^{*} \rightarrow \ell \nu \ell \nu$ decay mode with the ATLAS detector at $\sqrt{s}=13 \mathrm{TeV}$, ATLAS-CONF-2016-112.

[8] CMS collaboration, First results on Higgs to $W W$ at $\sqrt{s}=13 \mathrm{TeV}$, CMS-PAS-HIG-15-003.

[9] CMS collaboration, Observation of the Higgs boson decay to a pair of $\tau$ leptons with the CMS detector, Phys. Lett. B 779 (2018) 283 [arXiv:1708.00373] [InSPIRE].

[10] ATLAS collaboration, Cross-section measurements of the Higgs boson decaying to a pair of tau leptons in proton-proton collisions at $\sqrt{s}=13 \mathrm{TeV}$ with the ATLAS detector, ATLAS-CONF-2018-021.

[11] CMS collaboration, Observation of $\mathrm{t} \overline{\mathrm{t}} H$ production, Phys. Rev. Lett. 120 (2018) 231801 [arXiv: 1804.02610] [INSPIRE].

[12] ATLAS collaboration, Observation of Higgs boson production in association with a top quark pair at the LHC with the ATLAS detector, Phys. Lett. B 784 (2018) 173 [arXiv: 1806.00425] [INSPIRE].

[13] ATLAS collaboration, Observation of $H \rightarrow b \bar{b}$ decays and $V H$ production with the ATLAS detector, Phys. Lett. B 786 (2018) 59 [arXiv:1808.08238] [inSPIRE].

[14] CMS collaboration, Observation of Higgs boson decay to bottom quarks, Phys. Rev. Lett. 121 (2018) 121801 [arXiv:1808.08242] [INSPIRE]. 
[15] CMS collaboration, Search for a neutral MSSM Higgs boson decaying into tautau at 13 TeV, CMS-PAS-HIG-16-006.

[16] ATLAS collaboration, Search for the dimuon decay of the Higgs boson in pp collisions at $\sqrt{s}=13$ TeV with the ATLAS detector, ATLAS-CONF-2017-014.

[17] M. Carena, S. Gori, N.R. Shah and C.E.M. Wagner, A 125 GeV SM-like Higgs in the MSSM and the $\gamma \gamma$ rate, JHEP 03 (2012) 014 [arXiv: 1112.3336] [INSPIRE].

[18] A. Arbey, M. Battaglia, A. Djouadi, F. Mahmoudi and J. Quevillon, Implications of a 125 GeV Higgs for supersymmetric models, Phys. Lett. B 708 (2012) 162 [arXiv:1112.3028] [INSPIRE].

[19] H. Baer, V. Barger, P. Huang, D. Mickelson, A. Mustafayev and X. Tata, Post-LHCr fine-tuning in the minimal supergravity/CMSSM model with a 125 GeV Higgs boson, Phys. Rev. D 87 (2013) 035017 [arXiv: 1210.3019] [INSPIRE].

[20] A. Arbey, M. Battaglia, A. Djouadi and F. Mahmoudi, The Higgs sector of the phenomenological MSSM in the light of the Higgs boson discovery, JHEP 09 (2012) 107 [arXiv:1207.1348] [INSPIRE].

[21] W. Altmannshofer, M. Carena, N.R. Shah and F. Yu, Indirect Probes of the MSSM after the Higgs Discovery, JHEP 01 (2013) 160 [arXiv:1211.1976] [INSPIRE].

[22] K. Cheung, J.S. Lee and P.-Y. Tseng, Higgs Precision (Higgcision) Era begins, JHEP 05 (2013) 134 [arXiv: 1302.3794] [INSPIRE].

[23] D. Chowdhury and O. Eberhardt, Global fits of the two-loop renormalized Two-Higgs-Doublet model with soft $Z_{2}$ breaking, JHEP 11 (2015) 052 [arXiv:1503.08216] [INSPIRE].

[24] B. Bhattacherjee, A. Chakraborty and A. Choudhury, Status of the MSSM Higgs sector using global analysis and direct search bounds and future prospects at the High Luminosity LHC, Phys. Rev. D 92 (2015) 093007 [arXiv:1504.04308] [InSPIRE].

[25] P. Bechtle et al., The Light and Heavy Higgs Interpretation of the MSSM, Eur. Phys. J. C 77 (2017) 67 [arXiv: 1608.00638] [INSPIRE].

[26] P. Bechtle, S. Heinemeyer, O. Stal, T. Stefaniak, G. Weiglein and L. Zeune, MSSM Interpretations of the LHC Discovery: Light or Heavy Higgs?, Eur. Phys. J. C 73 (2013) 2354 [arXiv:1211.1955] [INSPIRE].

[27] A. Djouadi, Implications of the Higgs discovery for the MSSM, Eur. Phys. J. C 74 (2014) 2704 [arXiv: 1311.0720] [INSPIRE].

[28] P. Bechtle, S. Heinemeyer, O. Stal, T. Stefaniak and G. Weiglein, Applying Exclusion Likelihoods from LHC Searches to Extended Higgs Sectors, Eur. Phys. J. C 75 (2015) 421 [arXiv: 1507.06706] [INSPIRE].

[29] O. Buchmueller et al., The CMSSM and NUHM1 after LHC Run 1, Eur. Phys. J. C 74 (2014) 2922 [arXiv:1312.5250] [INSPIRE].

[30] S. Scopel, N. Fornengo and A. Bottino, Embedding the 125 GeV Higgs boson measured at the LHC in an effective MSSM: Possible implications for neutralino dark matter, Phys. Rev. D 88 (2013) 023506 [arXiv: 1304.5353] [INSPIRE].

[31] K.J. de Vries et al., The pMSSM10 after LHC Run 1, Eur. Phys. J. C 75 (2015) 422 [arXiv: 1504.03260] [INSPIRE]. 
[32] A. Barr and J. Liu, Analysing parameter space correlations of recent 13 TeV gluino and squark searches in the pMSSM, Eur. Phys. J. C 77 (2017) 202 [arXiv:1608.05379] [INSPIRE].

[33] K. Kowalska, Phenomenological MSSM in light of new 13 TeV LHC data, Eur. Phys. J. C 76 (2016) 684 [arXiv: 1608.02489] [inSPIRE].

[34] C. Han, J. Ren, L. Wu, J.M. Yang and M. Zhang, Top-squark in natural SUSY under current LHC run-2 data, Eur. Phys. J. C 77 (2017) 93 [arXiv: 1609.02361] [InSPIRE].

[35] M.R. Buckley, D. Feld, S. Macaluso, A. Monteux and D. Shih, Cornering Natural SUSY at LHC Run II and Beyond, JHEP 08 (2017) 115 [arXiv:1610.08059] [INSPIRE].

[36] J. Zhao, The Higgs properties in the MSSM after the LHC Run-2, Int. J. Mod. Phys. A 33 (2018) 1841001 [arXiv:1711.06461] [InSPIRE].

[37] E. Bagnaschi et al., Likelihood Analysis of Supersymmetric SU(5) GUTs, Eur. Phys. J. C 77 (2017) 104 [arXiv: 1610.10084] [INSPIRE].

[38] E. Bagnaschi et al., Likelihood Analysis of the Minimal AMSB Model, Eur. Phys. J. C 77 (2017) 268 [arXiv: 1612.05210] [INSPIRE].

[39] R.K. Barman, B. Bhattacherjee, A. Choudhury, D. Chowdhury, J. Lahiri and S. Ray, Current status of MSSM Higgs sector with LHC 13 TeV data, Eur. Phys. J. Plus 134 (2019) 150 [arXiv: 1608.02573] [INSPIRE].

[40] E. Bagnaschi et al., Likelihood Analysis of the pMSSM11 in Light of LHC 13-TeV Data, Eur. Phys. J. C 78 (2018) 256 [arXiv:1710.11091] [INSPIRE].

[41] J.C. Costa et al., Likelihood Analysis of the Sub-GUT MSSM in Light of LHC 13-TeV Data, Eur. Phys. J. C 78 (2018) 158 [arXiv:1711.00458] [INSPIRE].

[42] CMS collaboration, Sensitivity projections for Higgs boson properties measurements at the $H L-L H C$, CMS-PAS-FTR-18-011.

[43] ATLAS collaboration, Projections for measurements of Higgs boson cross sections, branching ratios, coupling parameters and mass with the ATLAS detector at the HL-LHC, ATL-PHYS-PUB-2018-054.

[44] M.E. Peskin, Comparison of LHC and ILC Capabilities for Higgs Boson Coupling Measurements, arXiv:1207.2516 [INSPIRE].

[45] M.E. Peskin, Estimation of LHC and ILC Capabilities for Precision Higgs Boson Coupling Measurements, in Proceedings, 2013 Community Summer Study on the Future of U.S. Particle Physics: Snowmass on the Mississippi (CSS2013), Minneapolis, MN, U.S.A., July 29-August 6, 2013 (2013) [arXiv: 1312.4974] [INSPIRE].

[46] ATLAS collaboration, Searches for Higgs boson pair production in the $h h \rightarrow b b \tau \tau, \gamma \gamma W W^{*}, \gamma \gamma b b, b b b b$ channels with the ATLAS detector, Phys. Rev. D 92 (2015) 092004 [arXiv: 1509.04670] [INSPIRE].

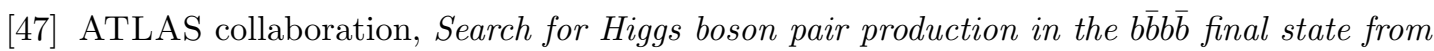
pp collisions at $\sqrt{s}=8 \mathrm{TeV}$ with the ATLAS detector, Eur. Phys. J. C $\mathbf{7 5}$ (2015) 412 [arXiv: 1506.00285] [INSPIRE].

[48] ATLAS collaboration, Search for pair production of Higgs bosons in the b $\bar{b} b \bar{b}$ final state using proton-proton collisions at $\sqrt{s}=13 \mathrm{TeV}$ with the ATLAS detector,

ATLAS-CONF-2016-049. 
[49] ATLAS collaboration, Search for Higgs boson pair production in the final state of $\gamma \gamma W W^{*}(\rightarrow l \nu j j)$ using $13.3 \mathrm{fb}^{-1}$ of pp collision data recorded at $\sqrt{s}=13 \mathrm{TeV}$ with the ATLAS detector, ATLAS-CONF-2016-071.

[50] ATLAS collaboration, Search for Higgs boson pair production in the $b \bar{b} \gamma \gamma$ final state using pp collision data at $\sqrt{s}=13$ TeV with the ATLAS detector, ATLAS-CONF-2016-004.

[51] CMS collaboration, Search for non-resonant Higgs boson pair production in the bbtautau final state using 2016 data, CMS-PAS-HIG-16-028.

[52] CMS collaboration, Search for resonant pair production of Higgs bosons decaying to two bottom quark-antiquark pairs in proton-proton collisions at $13 \mathrm{TeV}$, CMS-PAS-HIG-16-002.

[53] CMS collaboration, Search for heavy resonances decaying to a pair of Higgs bosons in four $b$ quark final state in proton-proton collisions at $\sqrt{s}=13 \mathrm{TeV}$, CMS-PAS-B2G-16-008.

[54] CMS collaboration, Search for $H(b \bar{b}) H(\gamma \gamma)$ decays at $\sqrt{s}=13 \mathrm{TeV}$, CMS-PAS-HIG-16-032.

[55] CMS collaboration, Search for resonant Higgs boson pair production in the $\mathrm{b} \overline{\mathrm{b}} \nu \mathrm{l} \nu$ final state at $\sqrt{s}=13 \mathrm{TeV}$, CMS-PAS-HIG-16-011.

[56] CMS collaboration, Search for pair production of Higgs bosons in the two tau leptons and two bottom quarks final state using proton-proton collisions at $\sqrt{s}=13 \mathrm{TeV}$, CMS-PAS-HIG-17-002.

[57] CMS collaboration, Search for resonant and non-resonant Higgs boson pair production in the $\mathrm{b} \overline{\mathrm{b}} \nu \mathrm{l} \nu$ final state at $\sqrt{s}=13 \mathrm{TeV}$, CMS-PAS-HIG-17-006.

[58] ATLAS collaboration, Search for pair production of Higgs bosons in the b $\bar{b} b \bar{b}$ final state using proton-proton collisions at $\sqrt{s}=13 \mathrm{TeV}$ with the ATLAS detector, Phys. Rev. D 94 (2016) 052002 [arXiv : 1606. 04782] [inSPIRE].

[59] ATLAS collaboration, Search for resonant and non-resonant Higgs boson pair production in the $b \bar{b} \tau^{+} \tau^{-}$decay channel in pp collisions at $\sqrt{s}=13 \mathrm{TeV}$ with the ATLAS detector, Phys. Rev. Lett. 121 (2018) 191801 [Erratum ibid. 122 (2019) 089901] [arXiv:1808.00336] [INSPIRE].

[60] ATLAS collaboration, Prospects for measuring Higgs pair production in the channel $H(\rightarrow \gamma \gamma) H(\rightarrow b \bar{b})$ using the ATLAS detector at the HL-LHC, ATL-PHYS-PUB-2014-019.

[61] ATLAS collaboration, Study of the double Higgs production channel $H(\rightarrow b \bar{b}) H(\rightarrow \gamma \gamma)$ with the ATLAS experiment at the HL-LHC, ATL-PHYS-PUB-2017-001.

[62] CMS collaboration, Updates on Projections of Physics Reach with the Upgraded CMS Detector for High Luminosity LHC, CERN-CMS-DP-2016-064.

[63] P. Osland and P.N. Pandita, Measuring the trilinear couplings of MSSM neutral Higgs bosons at high-energy $e^{+} e^{-}$colliders, Phys. Rev. D 59 (1999) 055013 [hep-ph/9806351] [INSPIRE].

[64] U. Baur, T. Plehn and D.L. Rainwater, Probing the Higgs selfcoupling at hadron colliders using rare decays, Phys. Rev. D 69 (2004) 053004 [hep-ph/0310056] [INSPIRE].

[65] J.-J. Liu, W.-G. Ma, G. Li, R.-Y. Zhang and H.-S. Hou, Higgs boson pair production in the little Higgs model at hadron collider, Phys. Rev. D 70 (2004) 015001 [hep-ph/0404171] [INSPIRE].

[66] C.O. Dib, R. Rosenfeld and A. Zerwekh, Double Higgs production and quadratic divergence cancellation in little Higgs models with T parity, JHEP 05 (2006) 074 [hep-ph/0509179] [INSPIRE]. 
[67] L. Wang, W. Wang, J.M. Yang and H. Zhang, Higgs-pair production in littlest Higgs model with T-parity, Phys. Rev. D 76 (2007) 017702 [arXiv:0705.3392] [INSPIRE].

[68] A. Pierce, J. Thaler and L.-T. Wang, Disentangling Dimension Six Operators through Di-Higgs Boson Production, JHEP 05 (2007) 070 [hep-ph/0609049] [INSPIRE].

[69] S. Kanemura and K. Tsumura, Effects of the anomalous Higgs couplings on the Higgs boson production at the Large Hadron Collider, Eur. Phys. J. C 63 (2009) 11 [arXiv:0810.0433] [INSPIRE].

[70] R. Contino, C. Grojean, M. Moretti, F. Piccinini and R. Rattazzi, Strong Double Higgs Production at the LHC, JHEP 05 (2010) 089 [arXiv: 1002.1011] [INSPIRE].

[71] R. Grober and M. Muhlleitner, Composite Higgs Boson Pair Production at the LHC, JHEP 06 (2011) 020 [arXiv: 1012.1562] [INSPIRE].

[72] M.J. Dolan, C. Englert and M. Spannowsky, New Physics in LHC Higgs boson pair production, Phys. Rev. D 87 (2013) 055002 [arXiv:1210.8166] [INSPIRE].

[73] M.J. Dolan, C. Englert and M. Spannowsky, Higgs self-coupling measurements at the LHC, JHEP 10 (2012) 112 [arXiv:1206.5001] [INSPIRE].

[74] G.D. Kribs and A. Martin, Enhanced di-Higgs Production through Light Colored Scalars, Phys. Rev. D 86 (2012) 095023 [arXiv: 1207.4496] [InSPIRE].

[75] R. Contino, M. Ghezzi, M. Moretti, G. Panico, F. Piccinini and A. Wulzer, Anomalous Couplings in Double Higgs Production, JHEP 08 (2012) 154 [arXiv:1205.5444] [INSPIRE].

[76] S. Dawson, E. Furlan and I. Lewis, Unravelling an extended quark sector through multiple Higgs production?, Phys. Rev. D 87 (2013) 014007 [arXiv: 1210.6663] [INSPIRE].

[77] U. Ellwanger, Higgs pair production in the NMSSM at the LHC, JHEP 08 (2013) 077 [arXiv: 1306.5541] [INSPIRE].

[78] K. Nishiwaki, S. Niyogi and A. Shivaji, ttH Anomalous Coupling in Double Higgs Production, JHEP 04 (2014) 011 [arXiv:1309.6907] [INSPIRE].

[79] A.J. Barr, M.J. Dolan, C. Englert and M. Spannowsky, Di-Higgs final states augMT2ed selecting hh events at the high luminosity LHC, Phys. Lett. B 728 (2014) 308 [arXiv: 1309.6318] [INSPIRE].

[80] N. Chen, C. Du, Y. Fang and L.-C. Lü, LHC Searches for The Heavy Higgs Boson via Two B Jets plus Diphoton, Phys. Rev. D 89 (2014) 115006 [arXiv:1312.7212] [InSPIRE].

[81] D.E. Ferreira de Lima, A. Papaefstathiou and M. Spannowsky, Standard model Higgs boson pair production in the $(b \bar{b})(b \bar{b})$ final state, JHEP 08 (2014) 030 [arXiv:1404.7139] [INSPIRE].

[82] C.-R. Chen and I. Low, Double take on new physics in double Higgs boson production, Phys. Rev. D 90 (2014) 013018 [arXiv: 1405.7040] [InSPIRE].

[83] J. Baglio, O. Eberhardt, U. Nierste and M. Wiebusch, Benchmarks for Higgs Pair Production and Heavy Higgs boson Searches in the Two-Higgs-Doublet Model of Type II, Phys. Rev. D 90 (2014) 015008 [arXiv:1403.1264] [InSPIRE].

[84] B. Hespel, D. Lopez-Val and E. Vryonidou, Higgs pair production via gluon fusion in the Two-Higgs-Doublet Model, JHEP 09 (2014) 124 [arXiv: 1407.0281] [INSPIRE].

[85] M. Slawinska, W. van den Wollenberg, B. van Eijk and S. Bentvelsen, Phenomenology of the trilinear Higgs coupling at proton-proton colliders, arXiv:1408.5010 [INSPIRE]. 


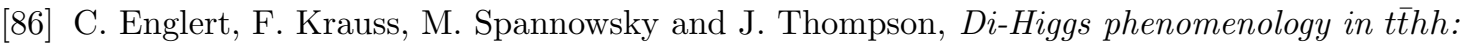
The forgotten channel, Phys. Lett. B 743 (2015) 93 [arXiv:1409.8074] [InSPIRE].

[87] F. Goertz, A. Papaefstathiou, L.L. Yang and J. Zurita, Higgs boson pair production in the $D=6$ extension of the SM, JHEP 04 (2015) 167 [arXiv:1410.3471] [INSPIRE].

[88] F. Maltoni, E. Vryonidou and M. Zaro, Top-quark mass effects in double and triple Higgs production in gluon-gluon fusion at NLO, JHEP 11 (2014) 079 [arXiv:1408.6542] [INSPIRE].

[89] A. Azatov, R. Contino, G. Panico and M. Son, Effective field theory analysis of double Higgs boson production via gluon fusion, Phys. Rev. D 92 (2015) 035001 [arXiv: 1502.00539] [INSPIRE].

[90] C.-T. Lu, J. Chang, K. Cheung and J.S. Lee, An exploratory study of Higgs-boson pair production, JHEP 08 (2015) 133 [arXiv:1505.00957] [INSPIRE].

[91] L.-C. Lü, C. Du, Y. Fang, H.-J. He and H. Zhang, Searching heavier Higgs boson via di-Higgs production at LHC Run-2, Phys. Lett. B 755 (2016) 509 [arXiv:1507.02644] [INSPIRE].

[92] A. Carvalho, M. Dall'Osso, T. Dorigo, F. Goertz, C.A. Gottardo and M. Tosi, Higgs Pair Production: Choosing Benchmarks With Cluster Analysis, JHEP 04 (2016) 126 [arXiv: 1507.02245] [INSPIRE].

[93] L. Bian and N. Chen, Higgs pair productions in the CP-violating two-Higgs-doublet model, JHEP 09 (2016) 069 [arXiv: 1607.02703] [INSPIRE].

[94] S. Banerjee, B. Batell and M. Spannowsky, Invisible decays in Higgs boson pair production, Phys. Rev. D 95 (2017) 035009 [arXiv: 1608.08601] [INSPIRE].

[95] M. Gorbahn and U. Haisch, Indirect probes of the trilinear Higgs coupling: $g g \rightarrow h$ and $h \rightarrow \gamma \gamma$, JHEP 10 (2016) 094 [arXiv: 1607.03773] [INSPIRE].

[96] A. Carvalho et al., Analytical parametrization and shape classification of anomalous HH production in the EFT approach, arXiv:1608.06578 [INSPIRE].

[97] Q.-H. Cao, G. Li, B. Yan, D.-M. Zhang and H. Zhang, Double Higgs production at the 14 TeV LHC and a $100 \mathrm{TeV}$ pp collider, Phys. Rev. D 96 (2017) 095031 [arXiv: 1611.09336] [INSPIRE].

[98] A. Crivellin, M. Ghezzi and M. Procura, Effective Field Theory with Two Higgs Doublets, JHEP 09 (2016) 160 [arXiv:1608.00975] [INSPIRE].

[99] R. Grober, M. Muhlleitner and M. Spira, Signs of Composite Higgs Pair Production at Next-to-Leading Order, JHEP 06 (2016) 080 [arXiv: 1602.05851] [INSPIRE].

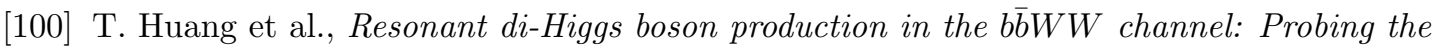
electroweak phase transition at the LHC, Phys. Rev. D 96 (2017) 035007 [arXiv: 1701.04442] [INSPIRE].

[101] G.D. Kribs, A. Maier, H. Rzehak, M. Spannowsky and P. Waite, Electroweak oblique parameters as a probe of the trilinear Higgs boson self-interaction, Phys. Rev. D 95 (2017) 093004 [arXiv: 1702.07678] [INSPIRE].

[102] L. Di Luzio, R. Gröber and M. Spannowsky, Maxi-sizing the trilinear Higgs self-coupling: how large could it be?, Eur. Phys. J. C 77 (2017) 788 [arXiv:1704.02311] [InSPIRE]. 
[103] K. Nakamura, K. Nishiwaki, K.-y. Oda, S.C. Park and Y. Yamamoto, Di-Higgs enhancement by neutral scalar as probe of new colored sector, Eur. Phys. J. C 77 (2017) 273 [arXiv: 1701.06137] [INSPIRE].

[104] A. Alves, T. Ghosh and K. Sinha, Can We Discover Double Higgs Production at the LHC?, Phys. Rev. D 96 (2017) 035022 [arXiv: 1704.07395] [INSPIRE].

[105] A. Adhikary, S. Banerjee, R.K. Barman, B. Bhattacherjee and S. Niyogi, Revisiting the non-resonant Higgs pair production at the HL-LHC, JHEP 07 (2018) 116 [arXiv: 1712.05346] [INSPIRE].

[106] M. Chala, C. Krause and G. Nardini, Signals of the electroweak phase transition at colliders and gravitational wave observatories, JHEP 07 (2018) 062 [arXiv: 1802.02168] [INSPIRE].

[107] P. Basler, S. Dawson, C. Englert and M. Mühlleitner, Showcasing HH production: Benchmarks for the LHC and HL-LHC, Phys. Rev. D 99 (2019) 055048 [arXiv: 1812.03542] [INSPIRE].

[108] T. Plehn and M. Rauch, The quartic Higgs coupling at hadron colliders, Phys. Rev. D 72 (2005) 053008 [hep-ph/0507321] [INSPIRE].

[109] T. Binoth, S. Karg, N. Kauer and R. Ruckl, Multi-Higgs boson production in the Standard Model and beyond, Phys. Rev. D 74 (2006) 113008 [hep-ph/0608057] [INSPIRE].

[110] W. Yao, Studies of measuring Higgs self-coupling with $H H \rightarrow b \bar{b} \gamma \gamma$ at the future hadron colliders, in Proceedings, 2013 Community Summer Study on the Future of U.S. Particle Physics: Snowmass on the Mississippi (CSS2013), Minneapolis, MN, U.S.A., July 29-August 6, 2013 (2013) [arXiv: 1308.6302] [INSPIRE].

[111] N. Liu, S. Hu, B. Yang and J. Han, Impact of top-Higgs couplings on Di-Higgs production at future colliders, JHEP 01 (2015) 008 [arXiv:1408.4191] [INSPIRE].

[112] A.J. Barr, M.J. Dolan, C. Englert, D.E. Ferreira de Lima and M. Spannowsky, Higgs Self-Coupling Measurements at a 100 TeV Hadron Collider, JHEP 02 (2015) 016 [arXiv: 1412.7154] [INSPIRE].

[113] H.-J. He, J. Ren and W. Yao, Probing new physics of cubic Higgs boson interaction via Higgs pair production at hadron colliders, Phys. Rev. D 93 (2016) 015003 [arXiv: 1506.03302] [INSPIRE].

[114] A. Papaefstathiou, Discovering Higgs boson pair production through rare final states at a $100 \mathrm{TeV}$ collider, Phys. Rev. D 91 (2015) 113016 [arXiv:1504.04621] [INSPIRE].

[115] A.V. Kotwal, M.J. Ramsey-Musolf, J.M. No and P. Winslow, Singlet-catalyzed electroweak phase transitions in the $100 \mathrm{TeV}$ frontier, Phys. Rev. D 94 (2016) 035022 [arXiv: 1605.06123] [INSPIRE].

[116] B. Fuks, J.H. Kim and S.J. Lee, Scrutinizing the Higgs quartic coupling at a future $100 \mathrm{TeV}$ proton-proton collider with taus and b-jets, Phys. Lett. B 771 (2017) 354 [arXiv: 1704.04298] [INSPIRE].

[117] S. Banerjee, C. Englert, M.L. Mangano, M. Selvaggi and M. Spannowsky, $h h+$ jet production at $100 \mathrm{TeV}$, Eur. Phys. J. C 78 (2018) 322 [arXiv: 1802.01607] [InSPIRE].

[118] E.W.N. Glover and J.J. van der Bij, Higgs boson pair production via gluon fusion, Nucl. Phys. B 309 (1988) 282 [INSPIRE]. 
[119] F. Boudjema and E. Chopin, Double Higgs production at the linear colliders and the probing of the Higgs selfcoupling, Z. Phys. C 73 (1996) 85 [hep-ph/9507396] [INSPIRE].

[120] T. Plehn, M. Spira and P.M. Zerwas, Pair production of neutral Higgs particles in gluon-gluon collisions, Nucl. Phys. B 479 (1996) 46 [Erratum ibid. B 531 (1998) 655] [hep-ph/9603205] [INSPIRE].

[121] A. Djouadi, W. Kilian, M. Muhlleitner and P.M. Zerwas, Production of neutral Higgs boson pairs at LHC, Eur. Phys. J. C 10 (1999) 45 [hep-ph/9904287] [InSPIRE].

[122] U. Baur, T. Plehn and D.L. Rainwater, Determining the Higgs Boson Selfcoupling at Hadron Colliders, Phys. Rev. D 67 (2003) 033003 [hep-ph/0211224] [INSPIRE].

[123] CMS collaboration, Search for Higgs boson pair production in events with two bottom quarks and two tau leptons in proton-proton collisions at $\sqrt{s}=13 \mathrm{TeV}$, Phys. Lett. B 778 (2018) 101 [arXiv: 1707.02909] [INSPIRE].

[124] CMS collaboration, Searches for a heavy scalar boson $H$ decaying to a pair of $125 \mathrm{GeV}$ Higgs bosons hh or for a heavy pseudoscalar boson A decaying to $\mathrm{Zh}$, in the final states with $h \rightarrow \tau \tau$, Phys. Lett. B 755 (2016) 217 [arXiv:1510.01181] [INSPIRE].

[125] ATLAS collaboration, Search for heavy resonances decaying into a $W$ or $Z$ boson and a Higgs boson in final states with leptons and b-jets in $36 \mathrm{fb}^{-1}$ of $\sqrt{s}=13$ TeV pp collisions with the ATLAS detector, JHEP 03 (2018) 174 [Erratum ibid. 11 (2018) 051] [arXiv: 1712.06518] [INSPIRE].

[126] M. Carena and Z. Liu, Challenges and opportunities for heavy scalar searches in the t $\bar{t}$ channel at the LHC, JHEP 11 (2016) 159 [arXiv:1608.07282] [INSPIRE].

[127] ATLAS collaboration, Search for Heavy Higgs Bosons A/H Decaying to a Top Quark Pair in pp Collisions at $\sqrt{s}=8 \mathrm{TeV}$ with the ATLAS Detector, Phys. Rev. Lett. 119 (2017) 191803 [arXiv: 1707.06025] [INSPIRE].

[128] CMS collaboration, Search for $t \bar{t}$ resonances in boosted semileptonic final states in $p p$ collisions at $\sqrt{s}=13 \mathrm{TeV}$, CMS-PAS-B2G-15-002.

[129] ATLAS collaboration, Search for heavy resonances decaying to $a W$ or $Z$ boson and a Higgs boson in the $q \bar{q}^{(\prime)} b \bar{b}$ final state in pp collisions at $\sqrt{s}=13 \mathrm{TeV}$ with the ATLAS detector, Phys. Lett. B 774 (2017) 494 [arXiv:1707.06958] [INSPIRE].

[130] ATLAS collaboration, Search for heavy particles decaying into top-quark pairs using lepton-plus-jets events in proton-proton collisions at $\sqrt{s}=13 \mathrm{TeV}$ with the ATLAS detector, Eur. Phys. J. C 78 (2018) 565 [arXiv:1804.10823] [INSPIRE].

[131] ATLAS collaboration, Combination of searches for heavy resonances decaying into bosonic and leptonic final states using $36 \mathrm{fb}^{-1}$ of proton-proton collision data at $\sqrt{\mathrm{s}}=13 \mathrm{TeV}$ with the ATLAS detector, Phys. Rev. D 98 (2018) 052008 [arXiv: 1808.02380] [INSPIRE].

[132] ATLAS collaboration, Search for additional heavy neutral Higgs and gauge bosons in the ditau final state produced in $36 \mathrm{fb}^{-1}$ of pp collisions at $\sqrt{s}=13 \mathrm{TeV}$ with the ATLAS detector, JHEP 01 (2018) 055 [arXiv:1709.07242] [INSPIRE].

[133] CMS collaboration, Search for nonresonant Higgs boson pair production in the $\mathrm{b} \overline{\mathrm{b}} \overline{\mathrm{b}}$ final state at $\sqrt{s}=13 \mathrm{TeV}$, JHEP 04 (2019) 112 [arXiv: 1810.11854] [INSPIRE].

[134] CMS collaboration, Search for resonant pair production of Higgs bosons decaying to bottom quark-antiquark pairs in proton-proton collisions at $13 \mathrm{TeV}$, CMS-PAS-HIG-17-009. 


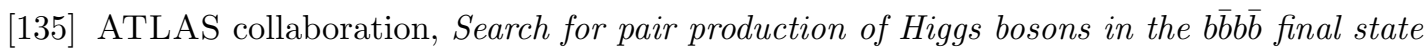
using proton-proton collisions at $\sqrt{s}=13 \mathrm{TeV}$ with the ATLAS detector, JHEP 01 (2019) 030 [arXiv: 1804.06174] [INSPIRE].

[136] CMS collaboration, Search for Higgs boson pair production in the final state containing two photons and two bottom quarks in proton-proton collisions at $\sqrt{s}=13 \mathrm{TeV}$, CMS-PAS-HIG-17-008.

[137] CMS collaboration, Search for Higgs boson pair production in the $\gamma \gamma \mathrm{b} \overline{\mathrm{b}}$ final state in $p p$ collisions at $\sqrt{s}=13 \mathrm{TeV}$, Phys. Lett. B 788 (2019) 7 [arXiv:1806.00408] [INSPIRE].

[138] ATLAS collaboration, Search for Higgs boson pair production in the $\gamma \gamma b \bar{b}$ final state with $13 \mathrm{TeV}$ pp collision data collected by the ATLAS experiment, JHEP 11 (2018) 040 [arXiv: 1807.04873] [INSPIRE].

[139] ATLAS collaboration, Search for Higgs boson pair production in the $\gamma \gamma W W^{*}$ channel using pp collision data recorded at $\sqrt{s}=13$ TeV with the ATLAS detector, Eur. Phys. J. C 78 (2018) 1007 [arXiv: 1807.08567] [InSPIRE].

[140] CMS collaboration, Search for resonant and nonresonant Higgs boson pair production in the $\mathrm{b} \overline{\mathrm{b}} \ell \nu \ell \nu$ final state in proton-proton collisions at $\sqrt{s}=13 \mathrm{TeV}$, JHEP 01 (2018) 054 [arXiv: 1708.04188] [INSPIRE].

[141] ATLAS collaboration, Search for Higgs boson pair production in the $b \bar{b} W W^{*}$ decay mode at $\sqrt{s}=13 \mathrm{TeV}$ with the ATLAS detector, JHEP 04 (2019) 092 [arXiv:1811.04671] [INSPIRE].

[142] ATLAS collaboration, Search for Higgs boson pair production in the $W W^{(*)} W W^{(*)}$ decay channel using ATLAS data recorded at $\sqrt{s}=13$ TeV, JHEP 05 (2019) 124 [arXiv: 1811.11028] [INSPIRE].

[143] J. Alwall et al., The automated computation of tree-level and next-to-leading order differential cross sections and their matching to parton shower simulations, JHEP 07 (2014) 079 [arXiv: 1405.0301] [INSPIRE].

[144] T. Sjöstrand, L. Lönnblad and S. Mrenna, PYTHIA 6.2: Physics and manual, hep-ph/0108264 [INSPIRE].

[145] CMS collaboration, Identification of heavy-flavour jets with the CMS detector in pp collisions at $13 \mathrm{TeV}, 2018$ JINST 13 P05011 [arXiv:1712.07158] [INSPIRE].

[146] DELPHES 3 collaboration, DELPHES 3, A modular framework for fast simulation of a generic collider experiment, JHEP 02 (2014) 057 [arXiv: 1307.6346] [INSPIRE].

[147] M.L. Mangano, M. Moretti, F. Piccinini and M. Treccani, Matching matrix elements and shower evolution for top-quark production in hadronic collisions, JHEP 01 (2007) 013 [hep-ph/0611129] [INSPIRE].

[148] https://twiki.cern.ch/twiki/bin/view/LHCPhysics/LHCHXSWGHH.

[149] https://twiki.cern.ch/twiki/bin/view/LHCPhysics/CERNYellowReportPageAt1314TeV2014.

[150] CMS collaboration, 2HDM Neutral Higgs Future Analysis Studies, CMS-PAS-FTR-13-024.

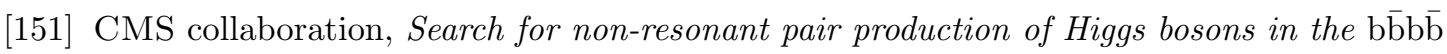
final state with $13 \mathrm{TeV}$ CMS data, CMS-PAS-HIG-16-026.

[152] ATLAS collaboration, Measurement prospects of the pair production and self-coupling of the Higgs boson with the ATLAS experiment at the HL-LHC, ATL-PHYS-PUB-2018-053. 
[153] https://twiki.cern.ch/twiki/bin/view/LHCPhysics/TtbarNNLO.

[154] G. Bagliesi, Tau tagging at ATLAS and CMS, in 17th Symposium on Hadron Collider Physics 2006 (HCP 2006), Durham, North Carolina, May 22-26, 2006 (2007) [arXiv: 0707.0928] [INSPIRE].

[155] T. Han, Collider phenomenology: Basic knowledge and techniques, in Physics in D $\geq 4$. Proceedings, Theoretical Advanced Study Institute in elementary particle physics, TASI 2004, Boulder, U.S.A., June 6-July 2, 2004, pp. 407-454 (2005) [DOI:10.1142/9789812773579_0008] [hep-ph/0508097] [INSPIRE].

[156] A. Lazopoulos, T. McElmurry, K. Melnikov and F. Petriello, Next-to-leading order QCD corrections to $t \bar{t} Z$ production at the LHC, Phys. Lett. B 666 (2008) 62 [arXiv:0804.2220] [INSPIRE].

[157] J.M. Campbell and R.K. Ellis, $t \bar{t} W^{+-}$production and decay at NLO, JHEP 07 (2012) 052 [arXiv: 1204.5678] [INSPIRE].

[158] S. Dittmaier, M. Krämer and M. Spira, Higgs radiation off bottom quarks at the Tevatron and the CERN LHC, Phys. Rev. D 70 (2004) 074010 [hep-ph/0309204] [INSPIRE].

[159] S. Dawson, C.B. Jackson, L. Reina and D. Wackeroth, Higgs production in association with bottom quarks at hadron colliders, Mod. Phys. Lett. A 21 (2006) 89 [hep-ph/0508293] [INSPIRE].

[160] S. Dawson, C.B. Jackson, L. Reina and D. Wackeroth, Higgs boson production with bottom quarks at hadron colliders, Int. J. Mod. Phys. A 20 (2005) 3353 [hep-ph/0409345] [INSPIRE].

[161] S. Dawson, C.B. Jackson, L. Reina and D. Wackeroth, Exclusive Higgs boson production with bottom quarks at hadron colliders, Phys. Rev. D 69 (2004) 074027 [hep-ph/0311067] [INSPIRE].

[162] R.V. Harlander and W.B. Kilgore, Higgs boson production in bottom quark fusion at next-to-next-to leading order, Phys. Rev. D 68 (2003) 013001 [hep-ph/0304035] [INSPIRE].

[163] S. Dawson, C.B. Jackson, L. Reina and D. Wackeroth, Higgs boson production with one bottom quark jet at hadron colliders, Phys. Rev. Lett. 94 (2005) 031802 [hep-ph/0408077] [INSPIRE].

[164] S. Dawson and P. Jaiswal, Weak Corrections to Associated Higgs-Bottom Quark Production, Phys. Rev. D 81 (2010) 073008 [arXiv:1002.2672] [INSPIRE].

[165] F. Maltoni, Z. Sullivan and S. Willenbrock, Higgs-Boson Production via Bottom-Quark Fusion, Phys. Rev. D 67 (2003) 093005 [hep-ph/0301033] [INSPIRE].

[166] E. Boos and T. Plehn, Higgs boson production induced by bottom quarks, Phys. Rev. D 69 (2004) 094005 [hep-ph/0304034] [INSPIRE].

[167] T. Plehn, Charged Higgs boson production in bottom gluon fusion, Phys. Rev. D 67 (2003) 014018 [hep-ph/0206121] [INSPIRE].

[168] R. Harlander, M. Krämer and M. Schumacher, Bottom-quark associated Higgs-boson production: reconciling the four- and five-flavour scheme approach, arXiv:1112.3478 [INSPIRE].

[169] A. Djouadi, L. Maiani, A. Polosa, J. Quevillon and V. Riquer, Fully covering the MSSM Higgs sector at the LHC, JHEP 06 (2015) 168 [arXiv: 1502.05653] [INSPIRE]. 
[170] F. Moortgat, S. Abdullin and D. Denegri, Observability of MSSM Higgs bosons via sparticle decay modes in CMS, hep-ph/0112046 [INSPIRE].

[171] G. Bélanger, D. Ghosh, R. Godbole and S. Kulkarni, Light stop in the MSSM after LHC Run 1, JHEP 09 (2015) 214 [arXiv:1506.00665] [INSPIRE].

[172] ATLAS collaboration, Search for top squarks decaying to tau sleptons in pp collisions at $\sqrt{s}=13$ TeV with the ATLAS detector, Phys. Rev. D 98 (2018) 032008 [arXiv: 1803.10178] [INSPIRE].

[173] ATLAS collaboration, Search for squarks and gluinos in final states with hadronically decaying $\tau$-leptons, jets and missing transverse momentum using pp collisions at $\sqrt{s}=13$ TeV with the ATLAS detector, Phys. Rev. D 99 (2019) 012009 [arXiv: 1808.06358] [INSPIRE].

[174] ATLAS collaboration, Search for new phenomena using the invariant mass distribution of same-flavour opposite-sign dilepton pairs in events with missing transverse momentum in $\sqrt{s}=13 \mathrm{TeV}$ pp collisions with the ATLAS detector, Eur. Phys. J. C 78 (2018) 625 [arXiv: 1805.11381] [INSPIRE].

[175] CMS collaboration, Search for supersymmetry in events with a photon, jets and missing transverse momentum in proton-proton collisions at $13 \mathrm{TeV}$, CMS-PAS-SUS-18-002.

[176] CMS collaboration, Inclusive search for supersymmetry using razor variables in $p p$ collisions at $\sqrt{s}=13 \mathrm{TeV}$, CMS-PAS-SUS-16-017.

[177] CMS collaboration, Search for supersymmetry using events with a photon, a lepton and missing transverse momentum in pp collisions at $\sqrt{s}=13 \mathrm{TeV}$, CMS-PAS-SUS-17-012.

[178] CMS collaboration, Search for Physics Beyond the Standard Model in Events with High-Momentum Higgs Bosons and Missing Transverse Momentum in Proton-Proton Collisions at $13 \mathrm{TeV}$, Phys. Rev. Lett. 120 (2018) 241801 [arXiv:1712.08501] [INSPIRE].

[179] R.-Y. Zhang, W.-G. Ma, L.-H. Wan and Y. Jiang, Supersymmetric electroweak corrections to the Higgs boson decays into chargino or neutralino pair, Phys. Rev. D 65 (2002) 075018 [hep-ph/0201132] [INSPIRE].

[180] T. Ibrahim, Neutralino decay of MSSM neutral Higgs bosons, Phys. Rev. D 77 (2008) 065028 [arXiv: 0803.4134] [INSPIRE].

[181] ATLAS collaboration, ATLAS searches for MSSM Higgs bosons decaying into SUSY cascades, ATL-PHYS-PUB-2009-079.

[182] S. Heinemeyer and C. Schappacher, Higgs Decays into Charginos and Neutralinos in the Complex MSSM: A Full One-Loop Analysis, Eur. Phys. J. C 75 (2015) 230 [arXiv: 1503. 02996] [INSPIRE].

[183] A.D. Medina and M.A. Schmidt, Enlarging Regions of the MSSM Parameter Space for Large $\tan \beta$ via SUSY Decays of the Heavy Higgs Bosons, JHEP 08 (2017) 095 [arXiv: 1706. 04994] [INSPIRE].

[184] S. Gori, Z. Liu and B. Shakya, Heavy Higgs as a Portal to the Supersymmetric Electroweak Sector, JHEP 04 (2019) 049 [arXiv:1811.11918] [INSPIRE].

[185] S. Kulkarni and L. Lechner, Characterizing simplified models for heavy Higgs decays to supersymmetric particles, arXiv:1711.00056 [INSPIRE]. 
[186] ATLAS collaboration, Search for pair production of higgsinos in final states with at least three b-tagged jets in $\sqrt{s}=13$ TeV pp collisions using the ATLAS detector, Phys. Rev. D 98 (2018) 092002 [arXiv: 1806.04030] [INSPIRE].

[187] ATLAS collaboration, Search for chargino-neutralino production using recursive jigsaw reconstruction in final states with two or three charged leptons in proton-proton collisions at $\sqrt{s}=13$ TeV with the ATLAS detector, Phys. Rev. D 98 (2018) 092012 [arXiv: 1806. 02293] [INSPIRE].

[188] ATLAS collaboration, Search for supersymmetry in events with four or more leptons in $\sqrt{s}=13$ TeV pp collisions with ATLAS, Phys. Rev. D 98 (2018) 032009 [arXiv: 1804.03602] [INSPIRE].

[189] ATLAS collaboration, Search for electroweak production of supersymmetric particles in final states with two or three leptons at $\sqrt{s}=13 \mathrm{TeV}$ with the ATLAS detector, Eur. Phys. J. C 78 (2018) 995 [arXiv: 1803.02762] [InSPIRE].

[190] ATLAS collaboration, Search for electroweak production of supersymmetric states in scenarios with compressed mass spectra at $\sqrt{s}=13 \mathrm{TeV}$ with the ATLAS detector, Phys. Rev. D 97 (2018) 052010 [arXiv: 1712.08119] [INSPIRE].

[191] ATLAS collaboration, Search for long-lived charginos based on a disappearing-track signature in pp collisions at $\sqrt{s}=13 \mathrm{TeV}$ with the ATLAS detector, JHEP 06 (2018) 022 [arXiv: 1712.02118] [INSPIRE].

[192] ATLAS collaboration, Search for the direct production of charginos and neutralinos in final states with tau leptons in $\sqrt{s}=13$ TeV pp collisions with the ATLAS detector, Eur. Phys.

J. C 78 (2018) 154 [arXiv:1708.07875] [INSPIRE].

[193] CMS collaboration, Searches for pair production of charginos and top squarks in final states with two oppositely charged leptons in proton-proton collisions at $\sqrt{s}=13 \mathrm{TeV}$, JHEP 11 (2018) 079 [arXiv: 1807.07799] [INSPIRE].

[194] CMS collaboration, Combined search for electroweak production of charginos and neutralinos in proton-proton collisions at $\sqrt{s}=13 \mathrm{TeV}$, JHEP 03 (2018) 160 [arXiv: 1801.03957] [INSPIRE].

[195] ATLAS collaboration, Search for heavy ZZ resonances in the $\ell^{+} \ell^{-} \ell^{+} \ell^{-}$and $\ell^{+} \ell^{-} \nu \bar{\nu}$ final states using proton-proton collisions at $\sqrt{s}=13 \mathrm{TeV}$ with the ATLAS detector, Eur. Phys. J. C 78 (2018) 293 [arXiv:1712.06386] [InSPIRE].

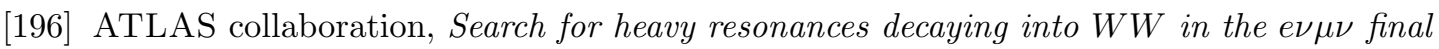
state in pp collisions at $\sqrt{s}=13 \mathrm{TeV}$ with the ATLAS detector, Eur. Phys. J. C 78 (2018) 24 [arXiv: 1710.01123] [INSPIRE].

[197] CMS collaboration, Search for additional neutral MSSM Higgs bosons in the di-tau final state in pp collisions at $\sqrt{s}=13 \mathrm{TeV}$, CMS-PAS-HIG-17-020.

[198] ATLAS collaboration, Search for new phenomena in high-mass diphoton final states using $37 \mathrm{fb}^{-1}$ of proton-proton collisions collected at $\sqrt{s}=13 \mathrm{TeV}$ with the ATLAS detector, Phys. Lett. B 775 (2017) 105 [arXiv: 1707.04147] [INSPIRE].

[199] ATLAS collaboration, Search for charged Higgs bosons decaying into top and bottom quarks at $\sqrt{s}=13 \mathrm{TeV}$ with the ATLAS detector, JHEP 11 (2018) 085 [arXiv:1808.03599] [INSPIRE]. 
[200] CMS collaboration, Combined measurements of Higgs boson couplings in proton-proton collisions at $\sqrt{s}=13$ TeV, Eur. Phys. J. C 79 (2019) 421 [arXiv:1809.10733] [InSPIRE].

[201] ATLAS collaboration, Measurements of gluon-gluon fusion and vector-boson fusion Higgs boson production cross-sections in the $H \rightarrow W W^{*} \rightarrow e \nu \mu \nu$ decay channel in pp collisions at $\sqrt{s}=13$ TeV with the ATLAS detector, Phys. Lett. B 789 (2019) 508 [arXiv:1808.09054] [INSPIRE].

[202] ATLAS collaboration, Combined measurements of Higgs boson production and decay in the $H \rightarrow Z Z^{*} \rightarrow 4 \ell$ and $H \rightarrow \gamma \gamma$ channels using $\sqrt{s}=13$ TeV pp collision data collected with the ATLAS experiment, ATLAS-CONF-2017-047.

[203] ATLAS collaboration, Measurements of Higgs boson properties in the diphoton decay channel with $36 \mathrm{fb}^{-1}$ of $p$ p collision data at $\sqrt{s}=13$ TeV with the ATLAS detector, Phys. Rev. D 98 (2018) 052005 [arXiv: 1802.04146] [INSPIRE].

[204] ATLAS collaboration, Search for Higgs bosons produced via vector-boson fusion and decaying into bottom quark pairs in $\sqrt{s}=13$ TeV pp collisions with the ATLAS detector, Phys. Rev. D 98 (2018) 052003 [arXiv: 1807.08639] [InSPIRE].

[205] ATLAS collaboration, Evidence for the $H \rightarrow b \bar{b}$ decay with the ATLAS detector, JHEP 12 (2017) 024 [arXiv: 1708.03299] [INSPIRE].

[206] ATLAS collaboration, Search for the standard model Higgs boson produced in association

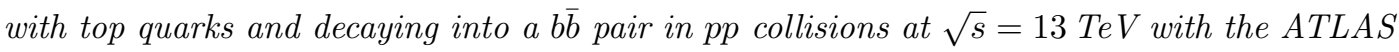
detector, Phys. Rev. D 97 (2018) 072016 [arXiv:1712.08895] [INSPIRE].

[207] ATLAS collaboration, Evidence for the associated production of the Higgs boson and a top quark pair with the ATLAS detector, Phys. Rev. D 97 (2018) 072003 [arXiv:1712.08891] [INSPIRE].

[208] S. Heinemeyer, W. Hollik and G. Weiglein, FeynHiggs: A Program for the calculation of the masses of the neutral CP even Higgs bosons in the MSSM, Comput. Phys. Commun. 124 (2000) 76 [hep-ph/9812320] [INSPIRE].

[209] B. Ananthanarayan, J. Lahiri and P.N. Pandita, Invisible decays of the heavier Higgs boson in the minimal supersymmetric standard model, Phys. Rev. D 91 (2015) 115025 [arXiv: 1507.01747] [INSPIRE].

[210] R.K. Barman, B. Bhattacherjee, A. Chakraborty and A. Choudhury, Study of MSSM heavy Higgs bosons decaying into charginos and neutralinos, Phys. Rev. D 94 (2016) 075013 [arXiv: 1607.00676] [INSPIRE].

[211] CMS collaboration, Performance of reconstruction and identification of tau leptons in their decays to hadrons and tau neutrino in LHC Run-2, CMS-PAS-TAU-16-002.

[212] M.A. Pickering, A search for neutral high-mass Higgs bosons decaying into pairs of hadronically decaying tau leptons in 13 TeV collisions recorded by the ATLAS detector, CERN-THESIS-2016-274.

[213] S. Wahrmund, Search for neutral MSSM Higgs bosons in the fully hadronic di-tau decay channel with the ATLAS detector, CERN-THESIS-2017-112.

[214] T. Sjöstrand et al., An Introduction to PYTHIA 8.2, Comput. Phys. Commun. 191 (2015) 159 [arXiv: 1410.3012] [INSPIRE].

[215] S. Dulat et al., New parton distribution functions from a global analysis of quantum chromodynamics, Phys. Rev. D 93 (2016) 033006 [arXiv:1506.07443] [INSPIRE]. 
[216] A.D. Martin, W.J. Stirling, R.S. Thorne and G. Watt, Parton distributions for the LHC, Eur. Phys. J. C 63 (2009) 189 [arXiv:0901.0002] [InSPIRE].

[217] H.-L. Lai et al., New parton distributions for collider physics, Phys. Rev. D 82 (2010) 074024 [arXiv: 1007.2241] [INSPIRE]. 